NBER WORKING PAPER SERIES

\title{
MEDICAL RESEARCH AND HEALTH CARE FINANCE: EVIDENCE FROM ACADEMIC MEDICAL CENTERS
}

\author{
Pierre Azoulay \\ Misty L. Heggeness \\ Jennifer L. Kao \\ Working Paper 27943 \\ http://www.nber.org/papers/w27943 \\ NATIONAL BUREAU OF ECONOMIC RESEARCH \\ 1050 Massachusetts Avenue \\ Cambridge, MA 02138 \\ October 2020, Revised September 2021
}

The authors thank participants at the 2020 ASSA Annual Meeting (San Diego), the NBER-IFS International Network on the Value of Medical Research Meeting, the NBER Productivity Lunch, the 2020 NBER Summer Institute, and UCLA. Amitabh Chandra and Adam Sacarny generously shared data for this project. This project was supported by the National Institute on Aging under Award Number R24AG048059 to the National Bureau of Economic Research. Soomi Kim provided excellent research assistance. Any opinions and conclusions expressed are those of the authors and do not represent the views of the US Census Bureau or the National Bureau of Economic Research. Address all correspondence to jennifer.kao@anderson.ucla.edu. All errors are our own.

NBER working papers are circulated for discussion and comment purposes. They have not been peer-reviewed or been subject to the review by the NBER Board of Directors that accompanies official NBER publications.

(C) 2020 by Pierre Azoulay, Misty L. Heggeness, and Jennifer L. Kao. All rights reserved. Short sections of text, not to exceed two paragraphs, may be quoted without explicit permission provided that full credit, including $(\odot$ notice, is given to the source. 
Medical Research and Health Care Finance: Evidence from Academic Medical Centers

Pierre Azoulay, Misty L. Heggeness, and Jennifer L. Kao

NBER Working Paper No. 27943

October 2020, Revised September 2021

JEL No. I13,I18,I23,O30

\section{ABSTRACT}

Academic Medical Centers (AMCs)—comprising medical schools, teaching hospitals, and research laboratories_-play an important role in US biomedical innovation. The Balanced Budget Act of 1997 changed reimbursements for Medicare inpatient claims and subsidies for medical residents. We compare AMCs' relative exposure to the reform and how these differences affect their researchers' ability to attract NIH grant funding, as well as the quantity, impact, and content of their publications. We find that in response to the reform, research activity increases by approximately $6 \%$, with larger effects observed for "translational" and clinical research. We find little effect on clinical outcomes.

Pierre Azoulay

MIT Sloan School of Management

100 Main Street, E62-487

Cambridge, MA 02142

and NBER

pazoulay@mit.edu

Misty L. Heggeness

U.S. Census Bureau

4600 Silver Hill Road

Washington, DC 20233

misty.1.heggeness@census.gov
Jennifer L. Kao

University of California, Los Angeles

Anderson School of Management

110 Westwood Plaza-D510

Los Angeles, CA 90095

jennifer.kao@anderson.ucla.edu 


\section{Introduction}

Research dating back at least to Solow (1956) has highlighted the role that innovation plays in driving economic growth. But the production of novel ideas is ultimately filtered through institutions that provide the incentive structure for knowledge accumulation (Dasgupta and David 1994; Mokyr 2002; Rosenberg 1963). The belief that basic research ultimately generates practical insights is perhaps the central assumption in post-war science policy, and that belief appears particularly well-founded in the health care industry (Gelijns and Rosenberg 1995; Cutler and McClellan 2001; Azoulay, Greenblatt, and Heggeness 2021). Yet in that setting and beyond, we have scant systematic understanding of the ways in which institutions - both in their formal (i.e., funding models, peer review) and informal (i.e., authorship and collaboration norms, tenure systems) aspects - support the transformation of scientific ideas into technological advances. ${ }^{1}$

In this paper, we crack open the institutional black box of Academic Medical Centers (AMCs), which, together with the National Institutes of Health (NIH) and the biopharmaceutical industry, play a central role in biomedical innovation. In the United States, thirty percent of health-related research is performed inside these institutions (Commonwealth Fund 1999), which bring together in one place a medical school, an owned or closely affiliated hospital, and basic research laboratories with an explicit triple mission of patient care, teaching, and research. Within the innovation system, AMCs are uniquely able to bring together the "ideas sector" of the health care economy (i.e., biomedical research) with its "production sector" (i.e., clinical care). This, in turn, facilitates the bidirectional flow of knowledge between the laboratory bench and the patient bedside (Rosenberg 2009).

We examine how research within AMCs is shaped by the impact of an external financing shock. Traditionally, financial support for their research mission comes from three different sources: grants from the NIH and private foundations, contracts with the pharmaceutical

\footnotetext{
${ }^{1}$ An important exception is Furman and Stern (2011), which examines the impact of biological resource centers on cumulative scientific discovery.
} 
industry, and importantly, cross-subsidies from patient care activities (Jones and Sanderson 1996). ${ }^{2}$ Building on recent empirical work that examines resource allocation within organizations (e.g., Giroud and Mueller 2019), we examine how a sudden decrease in institutional funding influences the rate, impact, and direction of research within AMCs.

Health care financing cuts have ambiguous effects on the level of subsequent research within AMCs. Cuts in reimbursement levels may encourage hospitals and physicians to substitute effort towards patient care activities and away from research. Low levels of crosssubsidies may also make it harder for the hospital to attract productive investigators, resulting in a net decrease in subsequent research levels. On the other hand, hospitals and physicians may reduce the level of patient care activities in response to a price reduction. Instead of providing patient care, hospitals and physicians may increase time spent on research, leading to a net increase in subsequent research intensity. The implications for the subsequent quality and direction of research are similarly ambiguous. For example, financing cuts may cause researchers to decrease both high- and low-risk projects, or alternatively to focus their attention towards research activities with greater impact.

We exploit quasi-experimental variation in cuts to clinical care revenues induced by the Balanced Budget Act of 1997 (BBA). The BBA was a major reform that led to considerable reductions in the level of Medicare reimbursements to hospitals (Seshamani, Schwartz, and Volpp 2006). Following a period of growth after the introduction of Medicare and Medicaid, the growth of clinical revenues slowed in the 1990s (Smith et al. 2005; Chen and Goldman 2016). This slowdown was partially due to increased federal efforts aimed at containing rising US health care expenditures, but also to other forces, such as the diffusion of managed care delivery models (Hellerstein 1998). Our analysis exploits the fact that the BBA decreased add-on payments made to support graduate medical education, suggesting that teaching hospitals were disproportionately affected by the reform. Among teaching hospitals, some

\footnotetext{
${ }^{2}$ Other funders include other federal agencies (e.g., the Centers for Disease Control and Prevention and the Agency for Healthcare Research and Quality) and state and local governments.
} 
institutions were harder hit by the reform than others because of differences in their reliance on Medicare patients as well as specific subsidies that increased the price they received from Medicare for the typical discharge (MedPAC 2003).

Our empirical analysis focuses on two samples of hospitals - one that includes all teaching hospitals and one that focuses on a research-intensive set of hospitals. We assemble a rich dataset that includes, for each hospital over the period 1992-2007, grant applications, funded grants, publications, and patient outcomes.

Using a difference-in-differences model that takes advantage of cross-hospital variation in the exposure to the reform, we find that cuts to hospital financing meaningfully increased subsequent research output. We show that hospitals most affected by the BBA experienced an approximately 6 percent increase in subsequent grant applications and funded grants. In particular, these effects are more pronounced for research activity conducted by "novice" investigators, relative to "incumbents" - those already ensconced in the peer review funding system. These findings stand in contrast with previous empirical work suggesting that restrictions to the funding environment can dampen subsequent research efforts (Furman, Murray, and Stern 2012; Tabakovic and Wollmann 2019), as well as with the pronouncements of academic medical leaders at the time of the reform (Iglehart 1999).

To further characterize the impact of these cuts, we examine the consequences of the $\mathrm{BBA}$ on the importance and composition of subsequent research activities. Measuring the impact of research is always fraught, and we analyze disparate effects of the reform along the "quality spectrum" using three separate measures: quantiles of the vintage-adjusted, articlelevel citation distribution, whether publications are cited in patent documents, and whether publications "disrupt" (or conversely consolidate) the prevailing scientific understanding and assumptions within a research subfield. In each case, we do not find evidence of heterogeneity along these dimensions, with the reform increasing low- and high-impact publication rates in a symmetric fashion. 
However, we do find that these increases are not evenly distributed across the vertical chain of biomedical research. Laboratory-based research articles appear largely unaffected, whereas "translational" research - which is geared towards bridging the gap between basic science discoveries and clinical applications, and difficult to perform outside the AMC setting - and clinical research - including clinical trials, which tend to be supported by industry funding - increase markedly in more exposed institutions, relative to less exposed ones. These results do not accord with practitioners' accounts (Meador 2015) and survey evidence (Weissman et al. 1999) pointing to the greater reliance of clinical investigators on institutional funds to support their research activities. On the contrary, our results are consistent with the view that researchers (or at least their employers) can "induce demand" in a manner similar to physicians shifting their practices' emphasis away from Medicare beneficiaries onto patients covered by private insurance (He and Mellor 2012).

As is traditionally the case for difference-in-differences research designs, a threat to identification could arise if the treatment variable is correlated with unobserved trends in the outcome. This concern is a salient one in our setting because Congress increased the NIH budget from $\$ 13.7$ billion to $\$ 27.2$ billion over this five-year period (Congressional Research Service 2020). It is plausible that that more research-intensive hospitals are more likely to benefit from the NIH budget doubling, for example because they have a research infrastructure in place that better enables them to scale up research activities. To address these concerns, our difference-in-differences specifications include separate trends for research-intensive and less research-intensive institutions. Though the magnitudes of the BBA effects are reduced, we continue to find a robust and sizable effect of the reform.

Finally, to better understand the overall consequences of the financing shock, we explore whether the BBA led physicians to substitute towards research, away from improving patient care activities. Looking at 30-day risk-adjusted survival rates across four conditions at the hospital level, we do not find any systematic association between BBA-exposure and 
subsequent clinical outcomes, suggesting that the positive impact of the BBA on subsequent research was not offset by worsening patient outcomes.

Our empirical analysis falls short of evaluating the overall welfare consequences of the BBA. Nonetheless, the positive impact on research investments in the medium term suggests that policy efforts aiming to decrease rents captured by health care providers do not unwittingly upset the delicate fabric of the biomedical research funding ecosystem.

The remainder of the paper proceeds as follows. Section II provides background information about AMCs and health care financing shocks. Section III describes the data. Section IV analyzes the effect of health care financing cuts on the rate, impact, and composition of subsequent research. Finally, Section V provides a discussion and concludes.

\section{Background and Conceptual Framework}

\section{A. Academic Medical Centers and Biomedical Research}

In the prototypical view of biomedical research, potential treatment discoveries undergo a sequential development process. First, researchers trained in the "basic" life sciences discover a new molecule and show that it inhibits a particular disease pathway in vitro. Then, they develop animal models and gather initial data on the molecule's safety and efficacy. The new molecule is subsequently turned over to physicians, who clinically test the purported treatment in randomized controlled trials. This stylized view underlies a broadbased congressional support for the continuous public funding of biomedical research and drives most of the policy discussion.

However, this linear model of innovation, "however flattering to the scientist and the academic, is economically naïve and simplistic in the extreme" (Rosenberg 1994: 139). Indeed, a closer examination of major treatment discoveries reveals a significantly more complex picture. In numerous cases, the first biological insight is acquired in a clinical setting, and only subsequently do bench scientists make sense of the mechanisms by which treatment is effective (Gershon 1998). For example, scientists discovered the first antidepressant drug, 
iproniazid, because a related compound used to treat tuberculosis made patients so euphoric that they stopped taking it. Subsequent research on iproniazid led to the chemical theories of depression that have generated all later antidepressant agents (Wurtman and Bettiker 1995).

In general, academic physicians have played an essential role in the development of various new medical technologies that rely on alternative development pathways. In some cases, such as the development of AIDS triple therapies, successful treatments resulted from the ongoing dialog between bench and bedside scientists (Wurtman 1997). Other times, new clinical uses are discovered for therapies already introduced into clinical practice (Gelijns and Rosenberg 1995; DeMonaco, Ali, and von Hippel 2012). Similarly, medical device users have also been instrumental in the invention of new products, from the identification of unmet clinical needs to builders of prototypes or initial field testing - a classic case of user-innovation (Gelijns and Rosenberg 1995). An extensive set of case studies lend credence to the belief that researchers within AMCs play an essential function in enabling these alternative pathways for treatment discovery, a belief strongly echoed by the academic medical establishment (Crowley and Gusella 2009).

For the past thirty years, research activities that focus on the bench-to-bedside interface have been labeled "translational." 3 Typically, translational research requires expertise in molecular biology, genetics, and a clinical subspecialty. As a result, it is often performed by physician-scientists who split their time between clinical care and research activities. Within that category of researchers, the relationship between patient care and research varies, ranging from individuals that are "interested in a disease mechanism and even occasionally interested in seeing patients," but "almost never interact in their research with an intact patient" to individuals that "actively search for patients who may enable them to uncover

\footnotetext{
${ }^{3}$ The Institute of Medicine's Clinical Research Roundtable defines translational research as the "transfer of new understandings of disease mechanisms gained in the laboratory into the development of new methods for diagnosis, therapy, and prevention and their first testing in humans" (Sung et al. 2003) though this definition does not appear to be widely agreed upon (Butler 2008; Woolf 2008).
} 
the secrets of complex diseases, care for those patients, and... undertake to explore new diagnoses and therapeutic approaches to treating their disease" (Nathan 2005).

Rather than sitting midpoint on a continuum stretching from fundamental research in biology all the way to the testing of novel therapeutics in large scale clinical trials, it is more appropriate to view translational research as belonging to "Pasteur's Quadrant," that is, in a class of investigations bringing forth ideas that are simultaneously valuable scientifically and a useful input into the treatment discovery process (Stokes 1997; Murray 2002). A well-known example is the work of Joseph Goldstein and Michael Brown, recipients of the 1985 Nobel Prize for Medicine and Physiology. Their initial investigations were inspired by observations of their own patients suffering from familial hypercholesterolemia (Goldstein and Brown 1997). Through patient-inspired basic investigations performed at the laboratory bench, they identified the underlying root case of this disease as a lack of lowdensity lipoprotein receptors. These discoveries in turn informed drug development efforts, ultimately leading to the market introduction of statins.

Distinguishing translational research from other types of biomedical research activities is not only more descriptively accurate, but also necessary to understand how wider changes in the health care system might affect the rate and direction of research efforts. First, translational research - which often focuses on uncovering the pathophysiologic mechanisms of disease (Ahrens 1992) - is very hard to appropriate, e.g., through patenting. As a result, its conduct is likely to be underfunded by private biopharmaceutical firms. Second, uniquely among the various types of biomedical research, translational activities are exceedingly difficult to perform outside of the AMC setting. In this respect they differ sharply from bench laboratory research, which often takes place in universities not affiliated with a medical school (e.g., MIT or UC Berkeley), independent research organizations (such as the Salk Institute) or pharmaceutical firms (Henderson 1994; Flier 2019). It also differs from clinical trial activities, which over the past three decades, have steadily migrated away from 
AMCs into a burgeoning ecosystem of for-profit experimental centers (Azoulay and Fishman 2020).

Consisting of a hospital, research laboratories, and a closely-affiliated medical school, AMCs bring together the wide range of resources, expertise, and personnel necessary to enable treatment discovery. ${ }^{4}$ They employ laboratory scientists, clinicians, physician-scientists, physicians-in-training, and graduate students. In addition, they attract a large number of patients with diverse phenotypes. This rare confluence entails that these institutions occupy a unique position in the biomedical research ecosystem, with a unique potential to facilitate the flow of knowledge from the laboratory bench to the patient's bedside (Rosenberg 2009; Ali and Gittelman 2016).

\section{B. $\quad$ Research Funding within Academic Medical Centers}

To support their research activities, AMCs primarily rely on three alternative sources of funding: grants (mostly from the National Institutes of Health, but also from other federal agencies, philanthropic foundations), contracts from biotechnology and pharmaceutical firms (including but not limited to the conduct of clinical trials), and "unsponsored" expenditures (i.e., lacking sponsorship from external sources, often a euphemism for cross-subsidies from clinical care).

NIH grants account for the largest share of research funding within AMCs - close to 70\%, or $\$ 7.4$ billion in 1997 (Commonwealth Fund 1999) - and AMCs capture more than two thirds of NIH extramural grant expenditures in a typical year. ${ }^{5}$ However, beginning in the 1980s, the mix of research supported by NIH steadily shifted towards laboratory investigations mostly conducted by scientists holding a PhD. ${ }^{6}$ The perceived lack of balance

\footnotetext{
${ }^{4}$ One strategy for identifying whether a hospital is "affiliated with a medical school" is if it reports a medical school affiliation to the American Medical Association. The American Hospital Association (AHA) Annual Survey indicates that more than 600 US hospitals were affiliated with a medical school in 2007 , the last year of our analysis. For a subset of the analyses that follow, we employ this definition to analyze the impact of the BBA on hospitals with high research and teaching intensity.

${ }^{5}$ Authors' tabulations using NIH's Compound Grant Applicant File.

${ }^{6}$ In 1995, PhD grantees outnumbered their MD counterparts by a ratio of $3: 1$, though application success rates for the two groups were similar (Nathan 1998). Furthermore, it is often suspected that successful MD
} 
has led to perennial calls by the academic medical establishment to protect funding for physician-scientists because they constitute an "endangered species" (Wyngaarden 1979; Rosenberg 1999; Jain et al. 2019). Over the past 25 years, task forces and blue ribbon commissions have suggested various avenues to reform peer review practices at NIH so as to avert a "crisis in clinical research" (Ahrens 1992; Nathan and Wilson 2003).

A second source of funding are contracts from industrial sponsors, typically (but not exclusively) for the conduct of clinical trials. ${ }^{7}$ Historically, AMCs' infrastructure, proximity to patients, and clinical expertise meant that they were the natural locus for the great bulk of clinical trials sponsored by industry. This began to change in the 1990s as a new crop of for-profit experimental centers started to compete with AMCs to provide biopharmaceutical sponsors with a more streamlined and lower-cost alternative (Azoulay and Fishman 2020). Private non-academic clinical trial sites came to partly replace their academic counterparts because the academic incentive system sometimes struggles to reward the conduct of research sponsored by firms. First, fellow academics sometimes view investigators with ties to industry as "tainted" (Prasad 2020); these perceptions have become heightened following a number of scandals involving human subjects protection and conflicts of interest (Stelfox et al. 1998; Baird, Downie, and Thompson 2002; Nathan and Weatherall 2002). Second, whereas investigator-initiated research makes unique demands on the creative and scientific potential of an academic, clinical trials involve a substantial relinquishing of intellectual autonomy since the investigator must adhere to an agreed-upon plan of research designed by others. As a result, participation in this activity does not produce career benefits commensurate with those generated by NIH-sponsored research - except, perhaps, for the "key opinion leader" recruited by industry to write the trial's clinical protocol.

The last substantial source of funding for research within AMCs falls under the nebu-

applicants share the scientific interests and basic research training of PhD-holding grantees, and do not necessarily interact directly with patients for research purposes (Ahrens 1992).

${ }^{7}$ Industry support accounted for 14\% of research expenditures within AMCs in 1997 (Commonwealth Fund 1999). 
lous umbrella of "unsponsored research." This funding includes expenditures by institutions or group practices, using rents from clinical care activities, endowment income, or institutional reserves, and by individual faculty members who devote their own resources to conduct research, drawing from discretionary funds or working additional uncompensated hours. These cross-subsidies are not systematically quantifiable, but survey evidence suggest that they play a non-negligible role in supporting AMCs' research mission. ${ }^{8}$ While much less important quantitatively than sponsored research expenditures, unsponsored expenditures might support the careers of young investigators struggling to establish their own independent program of research, or those of established scientists whose traditional sources of funding have temporarily ran out. Because faculty clinical practice plans are typically a major source of these expenditures, one might expect clinical researchers (which can include PhD-holding scientists working in clinical departments such as Internal Medicine or Pediatrics) to disproportionately benefit from this source of support.

In contrast with sponsored research, where proposals undergo stringent peer review (in the case of NIH grants) or must pass a market test (in the case of clinical trials), the welfare impact of unsponsored research activities is ambiguous. First, the existence of cross-subsidies means that AMCs earn rents from their clinical care activities, so that the same quality of care might have been delivered to patients at a lower cost (Nicholson and Song 2001). Second, the lack of transparency in the disbursement of institutional funds makes it susceptible to capture by entrenched interests, potentially resulting in low-quality or wasteful research by "hobby doctors." Our evidence will indirectly speak to this question, by examining the quantity, impact, and composition of research by institutions differentially exposed to financial stress.

\footnotetext{
${ }^{8}$ A survey conduced by the Association of American Medical Colleges found that 10 percent of the faculty-practice plan revenues were used to support research (Jones and Sanderson 1996); 43\% of AMC faculty members have reported receiving institutional funding for research (Weissman et al. 1999); and, in 1997, $9 \%$ of AMC research funding corresponded to support from faculty group practices.
} 


\section{Medicare Payments and Financing Cuts}

To provide plausibly exogenous variation in the extent of financial slack faced by AMCs and teaching hospitals, we rely on a measure of exposure to the Medicare reimbursement cuts triggered by the Balanced Budget Act of $1997 .{ }^{9}$

Under a system known as the Prospective Payment System, Medicare reimburses most hospitals on a per-admission basis (See Appendix A for a detailed discussion). In turn, each admission payment is a function of three types of adjustments: indirect medical education (IME) subsidies, disproportionate share hospital (DSH) payments, and outliers payments (Nicholson 2002). IME payments are meant to compensate teaching hospitals for indirect expenses stemming for example from use of diagnostic services by clinically inexperienced residents or decreased productivity of nurses and support staff involved in the teaching of residents. DSH payments correspond to payments received by hospitals for the additional cost of treating poor patients. Finally, outlier adjustments are reimbursements to compensate providers for patients with exceptionally costly stays (Keeler, Carter, and Trude 1988).

The BBA reduced the scale of all three adjustments and slowed the growth of payments for all diagnosis-related groups (See Appendix A for details). As a result, Medicare inpatient payments decreased by approximately five percent between 1998 and 2000 and many hospitals saw their financial status deteriorate significantly (Iglehart 1999; Dickler and Shaw 2000; Seshamani, Schwartz, and Volpp 2006; Shen and Wu 2013). Importantly, the reform was not anticipated, as its passage depended on the delicate balance of power between a reduced Republican majority in Congress and the Clinton administration following the November 1996 Federal election (Kahn and Kuttner 1999). ${ }^{10}$

\footnotetext{
${ }^{9}$ In the typical hospital, Medicare typically accounts for 30 percent of patient care revenues (Reinhardt 2006).

${ }^{10}$ After the passage of the reform, however, lobbying efforts by Medicare providers - with teaching hospitals in the frontline - proved successful in watering down some of its provisions: the Balanced Budget Refinement Act (BBRA) of 1998 slowed down the transition set by the BBA, a process continued by the Benefits Improvement and Protection Act (BIPA) of 2000 and the Medicare Modernization Act of 2003.
} 
As 30 percent of inpatient visits are funded by Medicare, cuts to Medicare reimbursements can represent a significant financial strain on hospitals. The effect of financial cuts on hospital activities has been widely studied in the realm of clinical care, with inconclusive results. In theory, hospitals may respond to reduced Medicare payments by cost-shifting-i.e., increasing prices for privately-insured patients - or cost-cutting-i.e., lowering hospital costs, decreasing support for unprofitable services (Cutler 1998, David et al. 2014). The empirical evidence to date has shown that, in response to adverse financial shocks, hospitals cut costs by limiting growth in hospital staff (Bazzoli et al. 2004) and lowering the quality of care (Lindrooth, Bazzoli, and Clement 2007). In contrast, Wu (2010) finds that in response to the BBA, hospitals are limited in their ability to shift costs to private payers (mostly due to the rise in influence of managed care delivery models during this period).

The consequences of financial stress for AMCs in particular are less well-understood, with no evidence to date regarding the impact on the research mission of these institutions. Financing cuts may shift research levels by influencing AMCs' hiring practices or exit rates among incumbent researchers. For example, hospitals may decrease the rate at which they hire faculty members or replace those that have left the institution; alternatively, hospitals may redirect their hiring efforts towards individuals that can more easily obtain funding from the government (i.e., laboratory bench researchers) or the private sector (i.e., trialists).

In addition to shaping research activity along the extensive margin, the BBA may shift research activity within individuals. For existing researchers, the net effect of financing cuts on research levels theoretically hinges on the relative strength of the substitution and income effects (McGuire and Pauly 1991; Yip 1998; Jacobson et al. 2010). If the former effect dominates, physician-investigators may substitute other career-advancing or other revenuegenerating activities - such industry-funded clinical trials - for clinical care. In this scenario, financing cuts would cause more easily-funded research activities to increase. If the income effect dominates, however, researchers may instead direct more effort towards patient care 
activities. In this setting, financing cuts may lower subsequent research levels, or alter the composition of the research portfolio, for individual researchers or the institution as a whole.

Qualitative accounts from physicians contemplating integrating a research component into their clinical practice often highlight the lumpy adjustment costs that need to be incurred in this process, with most expressing skepticism that their institutions could adequately support these efforts using the dwindling rents from clinical care activities. Because of the lack of fungibility between research and clinical care effort outside of the narrow context of clinical trials, our prior is that the extensive margin is likely to dominate and that any intensive margin (i.e., within-physician) substitution effects are likely to involve substitution of patient care or research activity towards "bedside" research-i.e., clinical trials.

Along both the extensive and intensive margins, however, our presumption is that the cuts induced by the BBA will disproportionately affect translational research - these investigations whose fundamental goal is to bridge the gap between the laboratory bench and the patient bedside.

\section{Data}

\section{A. Data Construction and Sources}

Our analysis combines data from several primary sources (i) hospital characteristics from the Healthcare Cost Report Information System (HCRIS) and the Inpatient Prospective Payment System (IPPS) Payment Impact Files; (ii) administrative data on NIH grants from the NIH IMPAC II database; (iii) publication and citation data from PubMed and the Web of Science, respectively; (iv) patent-to-publication citation linkages (from the USPTO and PubMed combined); and (v) hospital-level clinical outcomes data. Appendix Figure B1 describes how these data sources fit together and how we construct the variables used in this analysis.

We leverage information from HCRIS, which contains administrative data covering the 
universe of Medicare-certified hospitals. We identify hospitals with information from 1992 to 2007. For each hospital record, we observe hospital characteristics data, such as the total number of patient discharges, inpatient days, IME payments, and DSH payments. Medicare pricing information from the 1995 IPPS Payment Impact Files are used to calculate hospitals' average PPS revenue per discharge.

We supplement this hospital-level dataset with several measures of research activity. First, we link the hospital dataset to grants data from the NIH IMPAC II database. ${ }^{11}$ For each grant, we obtain information on investigators, their institutions, and a number of project characteristics. Next, we collect data on publications from PubMed, the publicaccess database which indexes the scientific literature, and we obtain publication citation data from the Web of Science (up to 2015).

A key challenge for estimating the causal impact of the BBA is that the level of the shock (changes to Medicare funding due to the BBA) and the measures of research outcomes (NIH grants and publications) do not coincide. An analysis that examines how Medicare payments at the individual hospital-level affects NIH grants allocated at the medical-school level is likely to produce estimates that suffer from measurement error. We overcome these challenges by employing an outcome assignment mechanism that uses principal investigator (or author) addresses to allocate each grant (or publication) to the "correct" hospital.

As an example, the University of California, San Francisco medical center includes the Parnassus Heights Campus, Mt. Zion Hospital and Medical Center, and San Francisco General Hospital (see Appendix Figure C1). Each of these locations has a unique Medicare provider number and therefore receives their own specific Medicare payments. However, the three campuses share a single, common NIH institutional code. Our strategy for matching hospitals to grants consists of looking at each of the PI addresses affiliated with the UCSF

\footnotetext{
${ }^{11}$ To construct the set of relevant grants, we limit our analysis to research project awards (NIH activity code $\mathrm{R}$ ), research career awards (NIH activity code $\mathrm{K}$ ), program projects and centers (NIH activity codes $\mathrm{M}$ and $\mathrm{P}$ ), cooperative agreements between NIH and a group of investigators (typically, NIH activity code $\mathrm{U} 01$ ), and R\&D contracts to evaluate a product or device (NIH activity code N01).
} 
NIH institutional code and allocating each PI (and grant) to one of the three hospitals.

We execute a similar strategy to match hospitals to publications: for each publication, we use the Web of Science to determine its authors' institutional affiliation and address. This allows us to match a publication to a hospital by matching on both hospital name and address. $^{12}$

The final sample consists of all hospitals that show evidence of teaching and research activity. To identify the set of research-active hospitals, we make several restrictions. First, we start with a list of 1,195 unique hospitals (as captured by unique Medicare provider numbers in the HCRIS data between 1992 and 2007). ${ }^{13}$ We drop 210 specialty hospitals as they are not paid under Medicare's Prospective Payment System, leading to a sample of 985 hospitals. ${ }^{14}$ Next, we exclude any hospitals that close during the 4 year period between 1992 and 1995 (the focal pre-BBA year in our analysis) by restricting our hospital sample to those with all observations between 1992 and 1995, resulting in 920 hospitals. ${ }^{15}$ Finally, to focus on a subset of hospitals for which the BBA is most likely to induce a meaningful shift in hospital outcomes, we restrict our sample to hospitals that receive (1) at least one indirect medical education payment and (2) produce at least one publication or submit at least one NIH grant application between 1992 and 2007. This results in a final sample consisting of 780 teaching hospitals (hereafter the "teaching hospital sample").

In addition to our primary hospital sample, we also consider a second, more researchfocused subset of the teaching hospital sample (the "AMC sample"). To create this second

\footnotetext{
${ }^{12}$ Notably, the Web of Science was launched in 1997. While the database contains records of articles published before 1997, the occurrence of missing author addresses is higher prior to 1998 (Liu, Hu, and Tang 2018). This does not threaten the validity of our estimates, since our regression specifications will include a full suite of calendar year effects.

${ }^{13}$ We address mergers and acquisitions over this time period by creating "super-hospitals" which inherit the Medicare patients, publication, and grants from their constituent units over the entire time period. Dropping these observations does not change our results.

${ }^{14}$ Specifically, "specialty hospitals" include: long term care, rehabilitation, psychiatric, pediatric, and cancer hospitals.

${ }^{15}$ Note that in the event that this subset of hospitals contains institutions whose closures were induced by the BBA, excluding this subset of hospitals may lead to an underestimate of the BBA's impact on hospital outcomes.
} 
hospital sample, we follow the definition of a "major teaching hospital" used by Burke et al. (2017). Major teaching hospitals are members of the Council of Teaching Hospitals (COTH) and have a medical school affiliation reported to the American Medical Association. ${ }^{16}$ This results in a sample of 274 hospitals.

The primary outcomes of interest are the total number of grant applications, grant awards, and publications that accrued to the researchers affiliated with a particular hospital in a given year. In addition, we characterize grants by grant cycle and principal investigator type. To assess how the BBA shapes research activity across principal investigators with varying levels of "grantsmanship" experience, we designate an application as an "incumbent" (respectively "novice") grant application if it comes from an applicants whose first application occurred at least three years (respectively within a window of three years) before the year of the focal application. ${ }^{17}$ When breaking down the number of funded applications for incumbents and novices, we measure whether their first award (not counting fellowship grants) occurred within a window of three years (for novices) or dates back from an even earlier period (for incumbents).

Further, we go beyond these raw counts by investigating how health care financing cuts shape the impact and composition of subsequent research. We rely on three measures of research impact. For our first measure of research impact, we assign each article to one of six mutually exclusive bins: those that fall in the bottom quartile of the article-level distribution of long-run citations, those that fall in the second quartile, in the third quartile, in the top quartile but not in the top ventile, in the top ventile but not the top percentile, and finally publications in the top percentile of the citation distribution. The percentile rankings are vintage-adjusted, which allows us to compare the citation impact of publications published in

\footnotetext{
${ }^{16}$ Hospital COTH status and AMA medical school affiliations are obtained from the American Hospital Association Annual Surveys from 1992 to 2007.

${ }^{17}$ In particular, we do not make use of NIH's "new" or "early-stage" investigator designations which aim to give scientists not yet ensconced in the peer review system a leg up. The reason is that these designations did not come into wide practice until the tail end of our observation period (cf. https://grants.nih.gov/policy/early-investigators/history.htm).
} 
different years. ${ }^{18}$ Our second measure of research impact comes from identifying the number of publications that were subsequently cited by a patent (Marx and Fuegi 2020).

Finally, our third measure of research impact comes from identifying each publications' "disruptive" impact using an index recently proposed by Funk and Owen-Smith (2017). Their citation-based measure captures the degree to which the ideas embodied in a paper consolidate or destabilize the scientific status quo. An article is considered "consolidating" if it tends to reference the same publications as the articles that will cite it in the future. Conversely, "disruptive" research draws on articles that will not be acknowledged by its own citing papers. For each hospital in the sample, we compute in every year the number of articles published that fall above the $95^{\text {th }}$ percentile of their index.

To investigate how the BBA shifts the allocation of research along the bench-to-bedside continuum, we construct measures of basic laboratory research (hereafter characterized as "laboratory" research), translational research, and clinical research. To partition the research space in this way, we take advantage of Medical Subject Headings (MeSH) terms, a hierarchical controlled vocabulary maintained by the National Library of Medicine. Most publications listed in PubMed are tagged with a set of MeSH terms, which characterize their scientific content. Using the MeSH-based definitions outlined in Azoulay, Greenblatt, and Heggeness (2021), we designate articles as bench research if they fulfill three criteria: (i) they are not disease-oriented (i.e., contain no disease MeSH terms); (ii) they do not report the results of a clinical trial (which we ascertain by examining the publication type field in PubMed); and (iii) they are tagged by MeSH terms denoting either the use of a molecular biology technique, the use a model organism, the study of cellular structures and macromolecules, or the study of biochemical and cellular processes.

\footnotetext{
${ }^{18} \mathrm{~A}$ vintage is comprised of all the articles published in a given year. To compute the quantiles of the vintage-specific, article-level distribution of citations, the relevant universe is not limited to the articles produced by researchers with addresses corresponding to the hospitals in our sample. Rather, the relevant universe includes the entire set of 17,312,059 articles that can be cross-linked between PubMed and the Web of Science.
} 
We next identify the set of publications that can plausibly be deemed to be translational in nature. Following Azoulay, Greenblatt, and Heggeness (2021), we generate three MeSHbased measures related to translational research. First, we label a publication translational if it is (i) disease-oriented; (ii) not a clinical trial; and (iii) also tagged by a basic science keyword used to describe bench research. Next, we denote a publication as "inspiring translational research" if it is translational and is cited by at least one clinical trial publication. Finally, we identify work that "builds on translational research," i.e., those that report the results of a clinical trial and list a translational publication in the reference list.

Our final measure of research outcomes is meant to capture research investments directly relevant for the bedside - i.e., clinical research. We focus on two measures: (i) clinical trial articles (identified using MeSH terms corresponding to clinical trials or the publication type field in PubMed) and (ii) "other" clinical articles, which are disease-oriented, not clinical trials, and not tagged by any bench MeSH keywords.

\section{B. Financial Pressure from the $B B A$}

To evaluate the impact of the BBA on subsequent research activity, we exploit the fact that some hospitals were more exposed to the reform than others. Following Shen (2003), we construct a "BBA Bite" variable that is the BBA-induced change in PPS revenue per discharge weighted by the share of Medicare patients in a base, pre-BBA year. As discussed in Section II.C, the BBA changed the formula for Medicare subsidy payments, which are a function of teaching load and the disproportionate share of poor patients. This suggests that crosshospital variation in the BBA's impact comes from differences in (i) hospital characteristics (e.g. the number of residents) and (ii) the hospital's reliance on Medicare.

To identify the effect of the BBA on PPS revenue per discharge, we rely on a simulated revenue approach. In particular, we use pre-BBA data to simulate the revenue that hospitals would have lost under the BBA, had the reform been in effect earlier. Using pre-BBA data isolates the mechanical effect of the policy from hospitals' endogenous responses. We simulate 
the average PPS revenue per discharge that hospitals would have received from Medicare had the BBA occurred in 1995, two years prior to its actual implementation. ${ }^{19}$ The mechanical effect of the BBA is estimated as the difference between the simulated revenue per discharge and actual PPS revenue per discharge in 1995:

$$
\operatorname{Sim} \Delta \operatorname{Rev}_{h, 1995}=\operatorname{Rev}_{h, 1995}-\operatorname{SimRev}_{h, 1995}
$$

where $R e v_{h, 1995}$ is the log of true PPS revenue per discharge in 1995 and $\operatorname{SimRev}_{h, 1995}$ is the log of simulated PPS revenue per discharge in 1995. Both revenue measures are averages across all Medicare discharges. Figure A1 shows that hospitals with a larger $\operatorname{Sim}_{\Delta} \operatorname{Rev}_{h}$ are more research-active in the pre-BBA era-a finding consistent with the expectation that research-active teaching hospitals are more likely to be disproportionately impacted by the BBA.

Finally, we weigh this measure by the share of a hospital's discharges that are reimbursed by Medicare in 1995. This follows previous literature which measures the financial impact of Medicare payment changes by using the hospital's base year Medicare discharge share (Acemoglu and Finkelstein 2008; Kaestner and Guardado 2008; Wu and Shen 2014). The overall BBA Bite is calculated as:

$$
B B A_{-} \text {Bite }_{h}=\left[\frac{\text { MedicareDischarges }}{\text { TotalDischarges }}\right]_{h, 1995} \times \operatorname{Sim}_{\text {Dimev }} \text { Rev }, 195
$$

The distribution of BBA Bite among hospitals in the teaching hospital sample is depicted in Figure 1. The figure shows that there is substantial variation in BBA Bite: the average BBA Bite is 0.0045 with a standard deviation of 0.0035 . Looking to the extremes of the distribution provide some illustrative examples: on the far left is South Bay Medical Center. In addition to having a low BBA Bite (0.00003), the California-based hospital has

\footnotetext{
${ }^{19}$ Specifically, we compute total IME and DSH payments keeping hospital inputs fixed at the 1995 level and using the 2001 IME and DSH payment formula.
} 
relatively low teaching and research intensity: the institution had three residents and produced one publication in 1997. In contrast, the St. Louis University Hospital - a similarly sized institution with a BBA Bite of 0.0184 - had more then 200 residents and produced 122 publications in the same year.

\section{Summary Statistics}

Table 1 provides hospital-level summary statistics for the teaching hospital sample. As can be seen in Panel A, the mean hospital has around 16,000 patient discharges, contains 400 beds, has 100 residents and interns, and reported roughly $\$ 10$ million in combined IME and DSH payments. Approximately a third of patient discharges correspond to Medicare patients. The average Medicare revenue per discharge is $\$ 8,000$.

Looking next to Panel B, the distributions of both the number of grant applications and the number of awarded grants are highly skewed (see Appendix Figure B2). For example, the mean number of grants awarded is roughly three, while one hospital (Massachusetts General Hospital) has nearly 150 grants funded during the period considered in our study. The majority of grant applications and awards correspond to new research proposals, as opposed to competitive renewals of already-funded grants.

Panel $\mathrm{C}$ shows that the average hospital had 46 publications in a year, but that this average also masks substantial variation - ranging from a minimum of 0 publications to a maximum of roughly 1,700 (see Appendix Figure B2). Among the publications produced, 4 percent were considered disruptive (as opposed to consolidating), there were similar rates (25 percent) of bench and translational research, and nearly 38 percent were considered clinical.

Across all hospitals, there is a marked increase in the level of grant applications (Appendix Figure B3) and grants funded (Appendix Figure B4) over our study period. The BBA occurred shortly before a sustained increase in the NIH budget, which concluded in 2003 (Korn et al. 2002; Freeman and Van Reenen 2009). Consequently, most of the teaching hos- 
pitals in our sample experienced an absolute increase in the grant funding awarded to their investigators, at least in the immediate aftermath of the shock. Our regression specifications will flexibly control for this secular increase through the inclusion of calendar year effects, making it possible to examine whether more exposed hospitals experience a relative decrease in research activity following the reform, relative to less exposed hospitals.

Appendix Figure B5 shows a similar upward trends for total publications. There is a discrete shift in 1998, which is likely explained by the use of the Web of Science in constructing the publication dataset, as discussed above. A more pronounced upward trend is observed for translational research and "other" clinical research articles, whereas the trajectories for laboratory research and clinical trails are relatively flat. ${ }^{20}$

Appendix Table D1 presents a comparison of the teaching hospital sample and the AMC sample. Relative to hospitals in the analysis hospital file, AMC sample hospitals produce significantly more grant applications and publications.

For a subset of the empirical exercises that follow, we examine the impact of hospital financing cuts on clinical outcomes in hospitals. Our main measures of clinical outcomes stem from Chandra et al.'s (2016) analysis of the relationship between hospital quality and market size. In particular, these authors construct hospital-level measures of 30-day riskadjusted survival for four frequent conditions: acute myocardial infarction (AMI), congestive heart failure, pneumonia, and hip/knee replacements (a common pair of surgical procedures). Specifically, they construct condition-specific measures using claims and survival outcomes in three-year rolling windows. ${ }^{21}$ Since the time period in the Chandra et al. (2016) study spans the pre- and post-BBA, periods, for each hospital we use the observation in 1996 (corresponding to an average over the pre-BBA years 1994, 1995, and 1996) and the observations (corresponding to an average over the post-BBA years 2000 through 2005). Of the

\footnotetext{
${ }^{20}$ For clinical trials, this likely reflects a general exodus of industry-sponsored activity away from academia during the period considered (Azoulay and Fishman 2020).

${ }^{21}$ For example, the, 30-day risk-adjusted survival for 1996 is calculated using patient claims during the years 1994, 1995, and 1996.
} 
780 hospitals in the teaching hospital sample, we successfully match 700 hospitals to the clinical outcomes dataset. Table 1, Panel D provides summary statistics for these survival outcomes, while Appendix Figure B6 presents histograms for the distribution of the survival rates for each of the conditions.

\section{Empirical Strategy and Results}

Our primary empirical strategy thus consists of comparing research outputs, before and after the implementation of the BBA, between hospitals that faced a potentially large decrease in the level of Medicare reimbursements (and have a relatively higher BBA Bite) to those that were minimally impacted by the reform (with a smaller BBA Bite):

$$
y_{h t}=\beta B B A_{-} \text {Bite }_{h} \times \operatorname{Post} B B A_{t}+\delta_{h}+\zeta_{t}+\varepsilon_{h t}
$$

where $y_{h t}$ is a measure of research outcome for hospital $h$ and year $t, A f \operatorname{ter} B B A_{t}$ is an indicator equal to 1 after 1997, $\delta_{h}$ are hospital fixed effects, and $\zeta_{t}$ are year fixed effects. ${ }^{22}$

One threat to identification is that our main results may biased by the doubling of the NIH budget between 1998 and 2003: research-intensive hospitals may have been more likely to benefit from the NIH budget increase (e.g., because they were most prepared to increase research production) and may have been more exposed to the BBA. ${ }^{23}$ To address these concerns, we include a proxy for hospital research intensity (RI): the average annual number of publications between 1990-1991 divided by $1000 .{ }^{24}$ The distribution of RI among hospitals in the teaching hospital sample is depicted in Figure 2. High RI hospitals have a higher BBA Bite (see Appendix Figure B7). We also find that hospitals with higher RI receive more indirect medical education and disproportionate share subsidies, have more

\footnotetext{
${ }^{22}$ In most cases we transform the outcome using the inverse hyperbolic sine transformation-asinh $(x)=$ $\log \left(x+\sqrt{x^{2}+1}\right)$ - to accommodate the large number of zero observations, while maintaining the ability to interpret the magnitude of coefficient estimates as elasticities (Burbidge, Magee and Robb 1988; Bellemare and Wichman 2020).

${ }^{23}$ For more details, see Appendix E.

${ }^{24}$ In robustness checks, we use an alternative, grant-based measure of hospital research intensity and find similar effects. See Appendix Table B1.
} 
residents, and produce more research (as measured by grant applications, grants funded, and publications) prior to the BBA (see Appendix Table B2).

Using this proxy for hospital research intensity, we modify equation (2) to directly estimate and control for RI hospitals' response to the NIH budget increase. Our main specification becomes:

$$
y_{h t}=\beta B B A_{-} \text {Bite }_{h} \times \text { PostBBA } A_{t}+\gamma R I_{h} \times \text { PostNIHDoubling } t+\delta_{h}+\zeta_{t}+\varepsilon_{h t}
$$

where $R I_{h}$ denotes the average number of publications between 1990-1991, PostNI H Doubling is an indicator equal to 1 after 1998, and all remaining variables are similar to those found in equation (2).

Table 2 examines the effect of the BBA when RI controls are excluded (odd columns) and included (even columns). To ease interpretation of the magnitudes, we present elasticities in the fifth row. Column 1 shows that there is a significant and positive effect of the BBA on grant applications. Column 2 reveals that these effects, though smaller in magnitude, remain significant and positive even after controlling for the doubling of the NIH budget: in particular, following the BBA's enactment, the total number of grant applications increases by 0.026 percent for each percent increase in BBA Bite. This is tantamount to a 6.1 percent increase in grant applications among hospitals whose BBA Bite is above the median (see Appendix Table B3).

Event studies that explore the timing of these estimated effects support these findings. In particular, we estimate:

$$
\begin{aligned}
Y_{h t}=\alpha+\sum_{z} \beta_{z} \times 1(z) \times H i g h_{-} B B A_{-} B_{i t e_{h}}+ \\
\sum_{w} \gamma_{w} \times 1(w) \times H i g h_{-} R I_{h}+\delta_{h}+\tau_{t}+\epsilon_{h t}
\end{aligned}
$$


where $H_{i g h \_B B A \_B i t e}$ and $H_{i g h}{ }_{-} I_{h}$ are indicators for whether a hospital $h$ 's BBA Bite and RI, respectively, are above the median, $\delta_{h}$ represents hospital fixed effects, and and $\tau_{t}$ represents year fixed effects. $z$ represents the "lag," or the years relative 1997, which is the year in which the BBA is implemented. $w$ represents the years relative to 1998, the year in which the NIH budget doubling begins.

Figure 3 plots the estimates of $\beta_{z}$ from equation (4) for total grant applications and corresponds to a dynamic version of Table B3, Columns 1 and 2. The figure also displays 95-percent confidence intervals and a dashed gray line that represents the year in which the BBA was enacted. The estimated coefficients illustrate that hospitals with high BBA Bites exhibit trends in grant applications similar to hospitals with low BBA Bite in years prior to the BBA. Further, using the methodology outlined in Roth (2021), we find little evidence that differential pre-trends (that we might be underpowered to detect) among high BBA Bite and low BBA Bite hospitals would substantially bias our results. ${ }^{25}$ Notably, the level of grant applications increases differentially in the wake of the BBA and continues to increase afterward. The increase in total grant applications is gradual, further providing evidence that these aggregate effects may mask significant heterogeneity in the response across different types of grants. In subsequent analyses, we provide additional evidence that hospitals respond to Medicare payment cuts through changes in their hiring practices.

Next, we examine the impact on the number of grants funded. We find similar results. Table 2, Column 2 and Figure B8 show that following the BBA, a percent increase in a hospital's BBA Bite is associated with an approximate 0.021 percent increase in the number of grants funded.

\footnotetext{
${ }^{25}$ Specifically, our analysis suggests that we could detect with 80 percent power a small linear positive trend of 0.014 and with 50 percent power a small linear positive trend of 0.009 . In regressions where we measure the impact of the BBA among High BBA hospitals, our average grant application estimates (0.059) are substantially larger (4.2x for 80 percent power, $6.6 \mathrm{x}$ for 50 percent power) than the potential bias. In the most extreme case, if a trend size of 0.014 exists, we calculate that it would lead to, by year 2 after the BBA, a bias of approximately 0.028. Our grant application event study estimates in year 2 are substantially larger $(2.5 \mathrm{x})$ than this potential bias.
} 
Turning to the effect on total publications, Table 2, Column 3 shows that the BBA has economically small and insignificant effects on the total number of subsequent publications. However, a challenge of only examining research aggregates is that AMCs and researchers may alter the nature of their research projects in response to financial strain, in which case examining hospital-level grant and publications total counts may mask how institutions and individuals reallocate their efforts among research projects with varying types and differing levels of impact. In the following sections, we turn to a more detailed investigation into how a hospital's research funding levels, impact, and compositional mix shifts in response to the BBA. All the specifications include the research intensity controls as in equations (3) and (4), though the corresponding estimates are not reported.

\section{A. Effects on Research Funding Levels}

Table 3 presents the estimates of the impact of the BBA on detailed measures of research funding levels, as measured by grant applications. In particular, we examine the impact of cuts in Medicare reimbursement on different types of grants (by grant cycle and principal investigator type). The estimates presented in Column 2 suggest that the positive response in total grant applications is driven by an increase in applications for new proposals. In contrast, there is no statistically significant effect on the number of "competing continuations," which correspond to applications seeking to extend previously awarded grants for an additional funding cycle.

Columns 4 and 5 show that the effect on total grant applications is driven primarily by an increase in those proposed by novices (rather than incumbents). Event studies echo these findings: grant applications rises more quickly and strongly among novices (see Appendix Figure B10). Taken together, these estimates signal that AMCs may respond to cuts to Medicare payments by turning to external grants as an additional source of funding. This can occur through two mechanisms: by encouraging existing researchers to seek additional grants and by hiring additional researchers who may "bring" grant funds with them. 
Estimates in Columns 6 through 8 explore the effects of the financing cuts on research conducted by MDs, PhDs, and MD-PhDs. In all cases, we estimate a strong, positive, and statistically significant effect of the BBA Bite on the level of subsequent grant applications. The PhD results are slightly larger in magnitude than the MD results: a one percent increase in BBA Bite yields a 0.034 percent increase in research conducted by PhD-holding principal investigators. In contrast, research conducted by MDs increases by 0.025 percent (though the difference between $\mathrm{PhD}$ and $\mathrm{MD}$ coefficients are not statistically significant). This accords with our priors: PhD-holding investigators are more likely to engage in research at the laboratory bench and MDs are traditionally associated with clinical and translational research. The finding that research conducted by MDs significantly increases can be explained by the fact that NIH grantees holding MD or MD-PhD degrees increasingly share similar scientific interests and methodological approaches with investigators who received traditional PhDs in the various disciplines within the life sciences domain (Ali and Gittelman 2016).

Appendix Table B4 demonstrate that similar patterns for grants funded persist when looking across grant cycle (new applications versus competing continuations) and principal investigator degree type.

\section{B. Effects on Research Impact}

Our results in Section IV.A suggest that the BBA significantly increased research conducted by novice rather than incumbent researchers, suggesting that hospitals responded to the Medicare cuts by adjusting hiring practices. However, an additional dimension through which hospitals may have responded to the BBA is by shifting the quality of subsequent research activities. Table 4 report the effects of the shock on publication rates adjusted for three measures of impact: publication-to-publication citation impact (Columns 2-7), patentto-publication citation impact (Columns 8-9), and "disruptiveness" (Columns 10-11).

Turning first to the effect on publication-to-publication citation impact and patent-topublication citation impact, much of the scholarship in the economics of innovation implicitly 
treats citation impact as a proxy for impact or "importance" (Hall, Jaffe, and Trajtenberg 2005; Azoulay, Graff Zivin, and Manso 2011), although this assumption has come under scrutiny (Funk and Owen-Smith 2017). If the decrease in publication volume documented above was driven by articles which do not make much of a ripple, leaving the impact on highly-cited articles unchanged, we might be tempted to conclude that the effect of the reform on research activities are at most second-order, relative to the effects on the cost and quality of health care delivery in these same institutions.

As described in Section III, publications are assigned to six mutually exclusive citationbased bins. We run the regression specified in equation (2) using each bin as a separate outcome. Looking across Columns 2 through 7, we cannot reject that the implied elasticities are essentially identical. We next examine the effect of the BBA on the number of publications that are subsequently cited by a patent, which we view as a proxy for the extent to which the work of AMC scientists shape biopharmaceutical firms' R\&D efforts. Columns 8 and 9 reveal similar effects across the number of publications that are and are not, respectively, cited by a patent. Overall, the evidence in Table 4 indicates that the effect of the reform are relatively homogeneous along the "citation as quality" dimension.

The remaining columns of Table 4 assess the extent to which the BBA disproportionately affects "disruptive" research. Using the $d$ index proposed by Funk and Owen-Smith (2017) to partition the set of articles for each hospital into "disruptive" publications ( $d$ in the top ventile) and "consolidating" publications ( $d \leq 95^{\text {th }}$ percentile). We find a slightly larger positive effect on disruptive research (Column 11) relative to consolidating research (Column 10), though the difference between the coefficients are not statistically significant. Taken together, the results suggest that the BBA ushers in an uniform increase in the level of both low- and high-impact scientific projects. 


\section{Effects on the Composition of the Research Portfolio}

Given the unique research capabilities and priorities of AMCs, a natural next question is to ask how the BBA shaped the composition of hospital research. Indeed, event study evidence hints that research aggregates may be masking compositional changes: Appendix Figure B9 shows that in the years immediately following the reform, the BBA has a significant and positive effect on the number of publications. This suggests that one consequence of allocating time towards more research is that existing AMC researchers may turn to the types of publications that were previously "waiting" in the file drawer.

In Table 5, we explore the possibility of compositional changes by examining the effect on laboratory research, translational, and clinical research. The results indicate that basic laboratory research is largely unaffected by the BBA: while a one percent increase in BBA Bite is associated with a 0.017 percent decrease in publications of this type. However, the estimates are statistically indistinguishable from 0 , likely due to the fact that our analysis is focused on hospitals that are primarily focused on translational and clinical research. Consistent with this view, we observe a quantitively and significantly more positive response for the level of translational research: the BBA causes translational research publications to increase by 0.024 percent for each percent increase in BBA Bite. This amplifying effect also extends to publications that "build on translational research" (Column 3) or "inspire translational research" (Column 4), both of which increase significantly more than translational research publications.

Looking next to clinical research publications, we find that the level of clinical research - as measured using the clinical trial definition (Column 5) and "other" clinical MeSHbased (Column 6) definition-increases substantially: a one percent increase in BBA Bite is associated with a 0.069 percent increase in clinical trial publications and 0.030 percent increase in "other" clinical research. As with translational research, the difference between changes in basic laboratory research and clinical research is statistically significant. 
In sum, the results presented in Table 5 paint a nuanced picture of compositional change in response to the health care financing cuts. The financing reform leaves bench research relatively unchanged, while triggering a sizable increase in investigations that can plausibly be labeled "translational" or "clinical." This runs counter to the qualitative accounts we gathered in interviews with academic leaders as well as "rank and file" clinical faculty members, which surmised that investigators who split their time between patient care and research activities would experience the effects of a reduction in cross-subsidies from their hospital's clinical care revenues more keenly.

\section{Countervailing Impact on Clinical Care}

Our results so far are consistent with the view that financial pressures might lead these scientists to allocate their time and effort away from patient care activities, towards research. As a result, the increase in subsequent research activity may be countered by declines in the quality of clinical care. A handful of previous studies have found that AMCs are associated with better outcomes (Keeler et al. 1992; Taylor, Whellan, and Sloan 1999; Ayanian and Weissman, 2002; Burke et al. 2017). In this section, we explore whether the BBA dampened these clinical care advantages through impacting patient outcomes within the hospitals in our sample.

We estimate the impact of the BBA on risk-adjusted survival rates in the "long difference" dimension of the data:

$$
\Delta Y_{h}^{c}=\beta B B A_{-} \text {Bite }_{h}+\text { Discharges }_{h}+\varepsilon_{h}
$$

where $\Delta Y_{h}^{c}$ corresponds to the change in the risk-adjusted 30-day mortality rate in hospital $h$ for condition $c$. Acting as a proxy for hospital size, Discharges $h_{h}$ is the log total number of patient discharges in 1995.

Table 6 reports the results. We find that, within this sample, the passage of the BBA 
does not adversely impact mortality outcomes following AMIs or heart failure episodes. Survival at 30-days is of course only a crude measure of care quality and the models have relatively low explanatory power, but it seems notable that we were unable to detect consistent declines in patient outcomes concurrently with the increase in research activity experienced by hospitals more exposed to the reform. ${ }^{26}$

\section{E. Robustness checks}

AMC Sample Analyses.-In Appendix D, we perform our analysis on the AMC sample, a subset of hospitals with high research and teaching intensity. This analysis reveals results that are fully consistent with our main results. In many cases, the estimated magnitudes are even greater than those found in the main teaching hospital sample, though in some cases, the estimates are not significant, largely due to the smaller sample size. For example, among research-intensive hospitals, a one percent increase in a hospital's BBA Bite translates into a 0.031 percent increase in the total number of subsequent grant applications (Appendix Table D2) and a 0.037 percent increase in the total number of grants funded (Appendix Table D3). We find a similar increase in the number of publications related to translational and clinical research (Appendix Table D5). As a robustness check, we confirmed that our results in the primary teaching hospital sample are not being driven by hospitals not found in the AMC sample.

Age Neutrality of the NIH Budget Doubling.-In our main specification, we address concerns that the NIH budget doubling may bias our results by including controls for hospital research intensity. The existing evidence demonstrates that positive and significant impact of the BBA on hospital research investments persist. However, a natural concern is that the increase in the NIH budget may still disproportionately affect hospitals that were more exposed to the BBA through a different channel - e.g., by targeting the type of research

\footnotetext{
${ }^{26}$ Most previous studies using samples not limited to teaching- and research-intensive hospitals find null to minimal impacts of the BBA on patient outcomes (Volpp et al. 2005; Seshamani, Schwartz, and Volpp 2006; Seshamani, Zhu, and Volpp, 2006). An exception is Wu and Shen (2014) who find that hospitals facing greater payment cuts experienced worse patient outcomes in the long-run.
} 
produced by hospitals most exposed to the reform. To investigate these concerns, we explore whether the doubling of the NIH budget was "age-biased." Empirically, we examine whether NIH budget increases disproportionately benefited research on health conditions affecting elderly populations. Medicare-eligible individuals are almost exclusively above 65 years old. Age-bias in the NIH research portfolio could advantage high-BBA Bite hospitals since their investigators have privileged access to patient populations relevant for the study of these conditions. Appendix Table E1 shows that funding appears to have flown slightly more towards research on diseases that targeted the elderly (vs. those that targeted the nonelderly), but the differences are small in magnitude and not statistically significant.

Subsidy BBA Bite Analysis.-In Appendix F, we examine the robustness of the core results previously presented in Section IV to an alternative measure of BBA Bite. Recall that hospital-level variation is driven by differences in the hospitals' reliance on Medicare payments and in particular, Medicare subsidy payments. In this robustness check, we measure of the BBA Bite as the share of 1995 hospital patient revenues that come from IME and DSH payments (Appendix Figure F1 graphs the distribution of this version of the BBA Bite). The regression results in Appendix Figures F1 through F4 are quite similar to those in Tables 3 through 5 , both in terms of magnitude of the elasticities and statistical significance. The corresponding event-study graphs, displayed in Appendix Figures F3 through F5 are similar to our core set of results. As described in Section III.B, we prefer measuring BBA Bite as the difference in simulated revenue per discharge and actual PPS revenue per discharge, weighed by the Medicare share of total discharges, as it allows us to more directly estimate the average hospital income loss as result of the BBA.

Instrumental Variables Estimation.-Appendix G considers an alternative strategy for capturing the exogenous variation in the PPS reimbursement formula. Following Shen (2003), we construct a simulated post-BBA revenue per discharge change that we use to instrument the actual post-BBA revenue per discharge change in a series of "long-difference" 
regressions. The difference in simulated and actual post-BBA revenue per discharge changes is documented in Appendix Figure G1. Table reports the two-stage least square regression results for our primary research outcomes. The results are consistent with our core results: hospitals that experience a larger financial loss as a result of the BBA experience an increase in research outcomes. The effects are both seen in the short-run (Panel A) and long-run (Panel B), though the effects are only statistically significant for publications in the long-run.

Intended Effects of the $B B A$.- Our primary analysis focuses on simulating the revenue per discharge that hospitals would have lost under the BBA, had the implemented changes been in effect in 1995. Notably, we focus on implemented changes to the Medicare payments, not the intended effects. However, as noted above, several post-BBA reforms were enacted to provide relief to hospitals, ultimately creating a wedge between the intended and implemented Medicare payment changes. To illustrate, DSH payments were reduced by two percent in 2001, rather than the four percent reduction as outlined in the BBA. We probe the robustness of our main results by simulating the revenue that hospitals would have lost under the BBA, had the intended effects of the reform been in effect earlier. The results in Appendix $\mathrm{H}$ are similar to the main results, both in terms of magnitude of the elasticities and statistical significance.

\section{Conclusion}

We investigate how Medicare financing cuts shape institutions that play a central role in the biomedical research ecosystem: Academic Medical Centers. Using a differences-in-difference approach, we find that research activities, measured using a variety of metrics, increase in response to a reform that decreases clinical care margins. Importantly, we document evidence that these effects do not cut evenly along the vertical chain of biomedical research. Laboratory-based "bench" research appears relatively unaffected. On the opposite end of the spectrum, clinical research activity (including industry-sponsored clinical trials) increases 
markedly in more exposed institutions, relative to less exposed ones. This is also the case for translational research, a type of "basic patient-oriented research" (Ahrens 1992) that is both hard to appropriate and difficult to perform outside of the AMC setting. Notably, we fail to find much evidence of countervailing impacts on the quality of care within these institutions.

For the past 30 years, McGuire and Pauly's (1991) model of physician behavior has provided health economists with a conceptual framework to analyze the effect of price changes for medical care in the presence of multiple payers. Our results can be interpreted through that lens: they are consistent with the substitution effect dominating the income effect, leading researchers to increase their research activities even though the returns from research have stayed relatively constant. However, this interpretation does not do justice to the subtleties of the academic medical setting. For example, in a competitive grant system (even one with rising paylines as in the immediate aftermath of the BBA passage), it is difficult for researchers to "induce demand" in a manner similar to a doctor treating Medicare patients or those covered by private insurance. Research activities are subject to lumpy adjustment costs and make the allocation of time less fractional than in the clinical care setting: a physician who stepped off the research funding treadmill might find herself unable to "prime the pump" of preliminary research results necessary to make her NIH grant proposals viable. An additional friction in the substitution process, and one regularly emphasized by academic medical leaders, is that lower Medicare reimbursements hampered AMCs' ability to crosssubsidize research activities. And yet, the combination of soft money appointments and relatively generous indirect cost recovery rates creates a channel for teaching hospitals and AMCs to quickly adjust to the financing shock-through the creation of new academic positions.

Our research design and data suffers from two principal limitations. First, we analyze the effect of the BBA at the level of the teaching and research hospital. This is sensible given 
the hospital-level nature of the financing shock, but leaves us unable to distinguish between impacts at the intensive-margin-e.g., within-individual substitution towards certain types of research activities - and impacts at the extensive margin - e.g., changes in AMC hiring practices or patterns of exit which favor clinical and translational researchers at the expense of scientists with a "basic" orientation.

Second, we can only speculate on how the BBA shock might differ from other types of financing shocks, such as a large philanthropic donation or endowment losses driven by the 2008 stock market collapse (Dranove, Garthwaite, and Ody 2017). Our sense is that "generic" shocks might well induce hospitals to increase or decrease research activities as a whole, but would not necessarily lead to the uneven effects we uncover. Because the BBA decreased slack financial resources on which a specific constituency - academic clinical departments - typically laid claim, the reform ended up shaping not simply the rate, but also the direction of research effort.

Our results inform the debate regarding the continued existence of Medicare price surcharges that favor teaching hospitals relative to other medical institutions (Nicholson 2002). Economists have typically been skeptical of the rationales offered in defense of these subsidies (Newhouse and Wilensky 2001; Grischkan, Friedman, and Chandra 2020), but a second-best argument in their favor is that the financial slack they create for AMCs could enable them to better support their social mission, including (but not limited to) research. The fact that the bottom did not fall out of translational research in the wake of the BBA, contrary to the dire pronouncements of academic medical leaders (Kassirer 1994) should lead us to regard with more skepticism self-interested claims that these subsidies must continue lest the delicate fabric of the biomedical research funding ecosystem gets torn apart.

Our empirical evidence on the effects of Medicare financing cuts demonstrate the importance of providing a more comprehensive and systematic analysis of the trade-offs in an institutional environment where seemingly disparate activities are tightly linked. As gov- 
ernments endeavor to reduce inefficiencies within the health care system, cost-containment initiatives may have positive or negative implications for institutions that occupy a central role in the biomedical research ecosystem. Attending to the innovation impacts of wellintentioned reform efforts is essential to ensure that new policies will in fact improve patient and societal welfare. 


\section{References}

Acemoglu, Daron and Amy Finkelstein. 2008. "Input and Technology Choices in Regulated Industries: Evidence from the Health Care Sector." Journal of Political Economy 116(5): 837-880.

Ahrens, Edward H. 1992. The Crisis in Clinical Research: Overcoming Institutional Obstacles. New York: Oxford University Press.

Ali, Ayfer and Michelle Gittelman. 2016. "Research Paradigms and Useful Inventions in Medicine: Patents and Licensing by Teams of Clinical and Basic Scientists in Academic Medical Centers." Research Policy 45(8): 1499-1511.

Ayanian, John Z. and Joel S. Weissman. 2002. "Teaching Hospitals and Quality of Care: A Review of the Literature." Milbank Quarterly 80(3): 569-593.

Azoulay, Pierre and Ariel Fishman. 2020. "Doctors, $\$ \$$ and Drug Development: The Rise of ForProfit Experimental Medicine." NBER Working Paper \#26892.

Azoulay, Pierre, Joshua S. Graff Zivin, and Gustavo Manso. 2011. "Incentive and Creativity: Evidence from the Academic Life Sciences." RAND Journal of Economics 42(3): 527-554.

Azoulay, Pierre, Wesley H. Greenblatt, and Misty L. Heggeness. 2020. "Long-term Effects from Early Exposure to Research: Evidence from the NIH 'Yellow Berets.'" Research Policy 50(9): 104332.

Baird, Patricia, Jocelyn Downie, and Jon Thompson. 2002. "Clinical Trials and Industry." Science 297(5590): 2211.

Bazzoli, Gloria J., Richard C. Lindrooth, Romana Hasnain-Wynia, and Jack Needleman. 2004. "The Balanced Budget Act of 1997 and US Hospital Operations." Inquiry 41(4): 401-417.

Bellemare, Marc F. and Casey J. Wichman. 2020. "Elasticities and the Inverse Hyperbolic Sine Transformation" Oxford Bulletin of Economics and Statistics 82(1): 50-61.

Burbidge, John B., Lonnie Magee, and A. Leslie Robb. 1988. "Alternative Transformations to Handle Extreme Values of the Dependent Variable," Journal of the American Statistical Association 83(401): 123-127.

Burke, Laura G., Austin B. Frakt, Dhruv Khullar, E. John Orav, and Ashish K. Jha. 2017. "Association Between Teaching Status and Mortality in US Hospitals." Journal of the American Medical Association 317(2):2105-2113.

Butler, Declan. 2008. "Translational Research: Crossing the Valley of Death." Nature 453(7197): 840-842.

Chandra, Amitabh, Amy Finkelstein, Adam Sacarny, and Chad Syverson. 2016. "Health Care Exceptionalism? Performance and Allocation in the US Health Care Sector." American Economic Review 106(8): 2110-2144.

Chen, Alice and Dana Goldman. 2016. "Health Care Spending: Historical Trends and New Directions." Annual Review of Economics 8: 291-319.

Commonwealth Fund Task Force on Academic Health Centers. 1999. From Bench to Bedside: Preserving the Research Mission of Academic Health Centers. New York.: The Commonwealth Fund. 
Congressional Research Service. 2020. National Institutes of Health (NIH) Funding: FY1994FY2020. Washington, DC: US Government Printing Office.

Cromwell, Jerry, Walter Adamache and Edward Drozd. 2006. "BBA Impacts on Hospital Residents, Finances, and Medicare Subsidies." Health Care Financing Review 28(1): 117129 .

Crowley, William F., Jr., and James F. Gusella. 2009. "Changing Models of Biomedical Research." Translational Research 1(1): 1-4.

Cutler, David M. and Mark McClellan. 2001. "Is Technological Change in Medicine Worth It?" Health Affairs 20(5): 11-29.

Cutler, David M. 1998. "Cost Shifting or Cost Cutting? The Incidence of Reductions in Medicare Payments." Tax Policy and the Economy 12: 1-27.

Dasgupta, Partha, and Paul A. David. 1994. "Toward a New Economics of Science." Research Policy 23(5): 487-521.

David, Guy, Richard C. Lindrooth, Lorens A. Helmchen, and Lawton R. Burns. 2014. "Do Hospitals Cross-Subsidize?" Journal of Health Economics 37: 198-218.

DeMonaco, Harold, Ayfer Ali, and von Hippel, Eric. 2012. "The Major Role of Clinicians in the Discovery of Off-Label Drug Therapies." Pharmacotherapy 26(3): 323-332.

Dickler, Robert and Gina Shaw. 2000. "The Balanced Budget Act of 1997: Its Impact on US Teaching Hospitals." Annals of Internal Medicine 132(10): 820-824.

Dranove, David, Craig Garthwaite, and Christopher Ody. 2017. "How Do Nonprofits Respond to Negative Wealth Shocks? The Impact of the 2008 Stock Market Collapse on Hospitals." RAND Journal of Economics 48(2): 485-525.

Flier, Jeffrey S. 2019. "Academia and Industry: Allocating Credit for Discovery and Development of New Therapies." Journal of Clinical Investigation 129(6): 2172-2174.

Freeman, Richard and John Van Reenen. 2009. "What if Congress Doubled R\&D Spending on the Physical Sciences?" In Innovation Policy and the Economy, edited by Josh Lerner and Scott Stern, 1-38. Chicago: University of Chicago Press.

Funk, Russell J. and Jason Owen-Smith. 2017. "A Dynamic Network Measure of Technological Change." Management Science 63(3): 791-817.

Furman, Jeffrey L. and Scott Stern. 2011. "Climbing atop the Shoulders of Giants: The Impact of Institutions on Cumulative Research." American Economic Review 101(5): 1933-1963.

Furman, Jeffrey L., Fiona Murray, and Scott Stern. 2012. "Growing Stem Cells: the Impact of Federal Funding Policy on the US Scientific Frontier." Journal of Policy Analysis and Management 31(3): 661-705.

Gelijns, Annetine C. and Nathan Rosenberg. 1995. "The Changing Nature of Technological Development." In Source of Medical Technology: University and Industry, edited by Nathan Rosenberg, Annetine C. Gelijns, and Holly Dawkins, 3-14. Washington, DC: The National Academies Press.

Gershon, Elliot S. 1998. "Making Progress: Does Clinical Research Lead to Breakthroughs in Basic Biomedical Sciences." Academic Medicine 42(1): 95-102.

Giroud, Xavier and Holger M. Mueller. 2019. "Firms' Internal Networks and Local Economic Shocks." American Economic Review 109(10): 3617-3649. 
Goldstein, Joseph L., and Michael S. Brown. 1997. "The Clinical Investigator: Bewitched, Bothered, and Bewildered-But Still Beloved." Journal of Clinical Investigation 99(12): $2803-2812$.

Grischkan, Justin A., Ari B. Friedman, and Amitabh Chandra. 2020. "Moving the Financing of Graduate Medical Education Into the 21 ${ }^{\text {st }}$ Century." Journal of American Medical Association 324(11): 1035-1036.

Hall, Bronwyn H., Adam Jaffe, and Manuel Trajtenberg. 2005. "Market Value and Patent Citations." The RAND Journal of Economics 36(1): 16-38.

He, Daifeng and Jennifer M. Mellor. 2012. "Hospital Volume Responses to Medicare's Outpatient Prospective Payment System: Evidence from Florida." Journal of Health Economics 31(5): 730-743.

Hellerstein, Judith. 1998. "Public Funds, Private Funds, and Medical Innovation: How Managed Care Affects Public Funds for Clinical Research." The American Economic Review 88(2): $112-116$

Henderson, Rebecca. 1994. "The Evolution of Integrative Capability: Innovation in Cardiovascular Drug Discovery." Industrial and Corporate Change 3(3): 607-630.

Iglehart, John K. 1999. "Support for Academic Medical Centers: Revisiting the 1997 Balanced Budget Act." New England Journal of Medicine 341(4): 299-304.

Jacobson, Mireille, Craig C. Earle, Mary Price, and Joseph P. Newhouse. 2010. "How Medicare's Payment Cuts for Cancer Chemotherapy Drugs Changed Patterns of Treatment." Health Affairs 29(7): 1391-1399.

Jain, Mukesh K., Vivian C. Cheung, Paul J. Utz, Brian K. Kobilka, Tadataka Yamada, and Robert Lefkowitz. 2019. "Saving the Endangered Physician-Scientist-A Plan for Accelerating Medical Breakthroughs." New England Journal of Medicine 381(5): 399-403.

Jones, Robert and Susan Sanderson. 1996. "Clinical Revenues Used to Support the Academic Mission of Medical Schools, 1992-93." Academic Medicine 71(3): 300-307.

Kaestner, Robert and Jose Guardado. 2008. "Medicare Reimbursement, Nurse Satffing, and Patient Outcomes." Journal of Health Economics 27(2): 339-361.

Kahn, Charles N., III., and Hanns Kuttner. 1999. Budget Bills and Medicare Policy: the Politics of the BBA. Health Affairs 18(1): 37-47.

Kassirer, Jerome P. 1994. "Academic Medical Centers Under Siege." New England Journal of Medicine 331(20): 1370-1371.

Keeler, Emmett B., Grace M. Carter, and Sally Trude. 1988. "Insurance Aspect of DRG Outlier Payments." Journal of Health Economics 7(3): 193-214.

Keeler, Emmett B., Lisa V. Rubenstein, Katherine L. Kahn, David Draper, Ellen R. Harrison, Michael J. McGinty, William H. Rogers, Robert H. Brook. 1992. "Hospital Characteristics and Quality of Care." Journal of the American Medical Association 268(13): 1709-1714.

Korn, David, Rich, Robert R., Garrison, Howard H., Golub, Sidney H., Hendrix, Mary J.C., Heinig, Stephen J., Masters, Bettie Sue, Turman, Richard J. 2002. "Science Policy : The NIH Budget in the 'Postdoubling' Era." Science 296(5572): 1401-1402.

Li, Danielle, Pierre Azoulay, and Bhaven N. Sampat. 2017. "The Applied Value of Public Investments in Biomedical Research." Science 356(6333): 78-81. 
Lindrooth, Richard C., Gloria J. Bazzoli, and Jan Clement. 2007. "The Effect of Reimbursement on the Intensity of Hospital Services." Southern Economic Journal 73(3): 575-87.

Liu, Weishu, Guangyuan Hu, and Li Tang. 2018. "Missing Author Address Information in Web of Science-An Explorative Study." Journal of Infometrics 12(3): 985-997.

Marx, Matt and Aaron Fuegi. 2020. "Reliance on Science: Worldwide Front-Page Patent Citations to Scientific Articles." Strategic Management Journal 41(): 1-23.

McGuire, Thomas G. and Mark V. Pauly. 1991. "Physician Response to Fee Changes with Multiple Payers." Journal of Health Economics 10(4): 385-410.

Meador, Kimford J. 2015. "Decline of Clinical Research in Academic Medical Centers." Neurology 85(13): 1171-1176.

Medicare Payment Advisory Commission. 2003. Report to Congress: Medicare Payment Policy. Washington, DC: Medicare Payment Advisory Commission

Mokyr, Joel. 2002. The Gifts of Athena: Historical Origins of the Knowledge Economy. Princeton: Princeton University Press.

Murray, Fiona. 2002. "Innovation as Co-evolution of Scientific and Technological Networks: Exploring Tissue Engineering." Research Policy 31(8-9): 1389-1403.

Nathan, David G. 1998. "Clinical Research: Perceptions, Reality, and Proposed Solutions." Journal of the American Medical Association 280(16): 1427-1431.

Nathan, David G. 2005. "The Several Cs of Translational Clinical Research." Journal of Clinical Investigation 115(4): 795-797.

Nathan, David G. and David J. Weatherall. 2002. "Academic Freedom in Clinical Research." New England Journal of Medicine 347(17): 1368-1371.

Nathan, David G. and Jean D. Wilson. 2003. "Clinical Research and the NIH-A Report Card." New England Journal of Medicine 349(19): 1860-1865.

Newhouse, Joseph P. and Gail R. Wilensky. 2001. "Paying For Graduate Medical Education: The Debate Goes On." Health Affairs 20(2): 136-147.

Nicholson, Sean. 2002. Medicare Hospital Subsidies. Washington, DC: The AEI Press.

Nicholson, Sean and David Song. 2001. "The Incentive Effects of the Medicare Indirect Medical Education Policy." Journal of Health Economics 20(6): 909-933.

Prasad, Vinayak K. 2020. How Bad Policy and Bad Evidence Harm People with Cancer. Baltimore, MD: Johns Hopkins University Press.

Reinhardt, Uwe E. 2006. "The Pricing of US Hospital Services: Chaos Behind a Veil of Secrecy." Health Affairs 25(1): 57-69.

Rosenberg, Leon E. 1999. "Physician-Scientists_Endangered and Essential." Science 283(5400): 331-332.

Rosenberg, Nathan. 1963. Technological Change in the Machine Tool Industry, 1840-1910. Journal of Economic History. 23(4): 414-443.

Rosenberg, Nathan. 1994. Exploring the Black Box. New York: Cambridge University Press.

Rosenberg, Nathan. 2009. "Some Critical Episodes in the Progress of Medical Innovation: An Anglo-American Perspective." Research Policy 38(2): 234-242. 
Roth, Jonathan. 2021. "Pre-test with Caution: Event-study Estimates After Testing for Parallel Trends." Working Paper.

Seshamani, Meena, J. Sanford Schwartz, and Kevin G. Volpp. 2006. "The Effect of Cuts in Medicare Reimbursement on Hospital Mortality." Health Services Research 41(3 Pt 1): 683700 .

Seshamani, Meena, Jingsan Zhu, and Kevin G. Volpp. 2006. "Did Postoperative Mortality Increase after the Implementation of the Medicare Balanced Budget Act?" Medical Care 44(6): 527533.

Shen, Yu-Chu. 2003. "The Effect of Financial Pressure on the Quality of Care in Hospitals." Journal of Health Economics 22(2): 243-269.

Shen, Yu-Chu and Vivian Y. Wu. 2013. "Reductions in Medicare Payments and Patient Outcomes: An Analysis of 5 Leading Medicare Conditions." Medical Care 51(11): 970-977.

Smith, Cynthia, Cathy Cowan, Art Sensenig, Aaron Caitlin, and the Health Accounts Team. 2005. "Health Spending Growth Slows in 2003." Health Affairs 24(1): 185-194.

Solow, Robert M. 1956. "A Contribution to the Theory of Economic Growth." Quarterly Journal of Economics 70(1): 65-94.

Stelfox, Henry Thomas, Grace Chua, Keith O'Rourke, and Allan Detsky. 1998. "Conflict of Interest in the Debate Over Calcium-Channel Antagonists." New England Journal of Medicine 338(2): 101-106.

Stokes, Donald E. 1997. Pasteur's Quadrant: Basic Science and Technological Innovation. Washington, DC: Brookings Institution Press.

Sung, Nancy S., William F. Crowley, Jr., Myron Genel, et al. 2003. "Central Challenges Facing the National Clinical Research Enterprise." Journal of the American Medical Association 289(10): 1278-1287.

Taylor, Donald H., David J. Whellan, and Frank A. Sloan. 1999. "Effects of Admission to a Teaching Hospital on the Cost and Quality of Care for Medicare Beneficiaries." New England Journal of Medicine 340(4): 293-299.

Tabakovic, Haris and Thomas Wollmann. 2019. "The Impact of Money on Science: Evidence from Unexpected NCAA Football Outcomes." Journal of Public Economics 178: 104066

Trajtenberg, Manuel, Rebecca Henderson, and Adam Jaffe. 1997. "University vs. Corporate Patents: A Window on the Basicness of Innovations." Economics of Innovation and New Technology 5(1): 19-50.

Volpp, Kevin G., R. Tamara Konetzka, Jingsan Zhu, Lori Parsons, and Eric Peterson. 2005. "Effect of Cuts in Medicare Reimbursement on Process and Outcome of Care for Acute Myocardial Infarction Patients?" Circulation 112(15): 2268-2275.

Weissman, Joel S., Demet Saglam, Eric G. Campbell, Nancyanne Causino, and David Blumenthal. 1999. Journal of American Medical Association 281(12): 1093-1098.

Woolf, Steven H. 2008. "The Meaning of Translational Research and Why It Matters." Journal of American Medical Association 299(2): 211-2013.

Wu, Vivian. 2010. "Hospital Cost-shifting Revisited: New Evidence from the Balanced Budget Act of 1997." International Journal of Health Care Finance and Economics 28(2): 350-360. 
Wu, Vivian Y. and Yu-Chu Shen. 2014. "Long-Term Impact of Medicare Payment Reductions on Patient Outcomes." Health Services Research 49(5):1596-1615.

Wurtman, Richard J., and Robert L. Bettiker. 1995. "The Slowing of Treatment Discovery." Nature Medicine 1(11): 1122-1125.

Wurtman, Richard J. 1997. "What Went Right: Why is HIV a Treatable Infection?" Nature Medicine 3(7): 714-717.

Wyngaarden, James B. 1979. "The Clinical Investigator as an Endangered Species." New England Journal of Medicine 301(23): 1254-1259.

Yip, Winnie C. 1998. "Physician Response to Medicare Fee Reductions: Changes in the Volume of CABG Surgeries in the Medicare and Private Sectors." Journal of Health Economics 17(6): 675-699. 
Figure 1: Distribution of BBA Bite

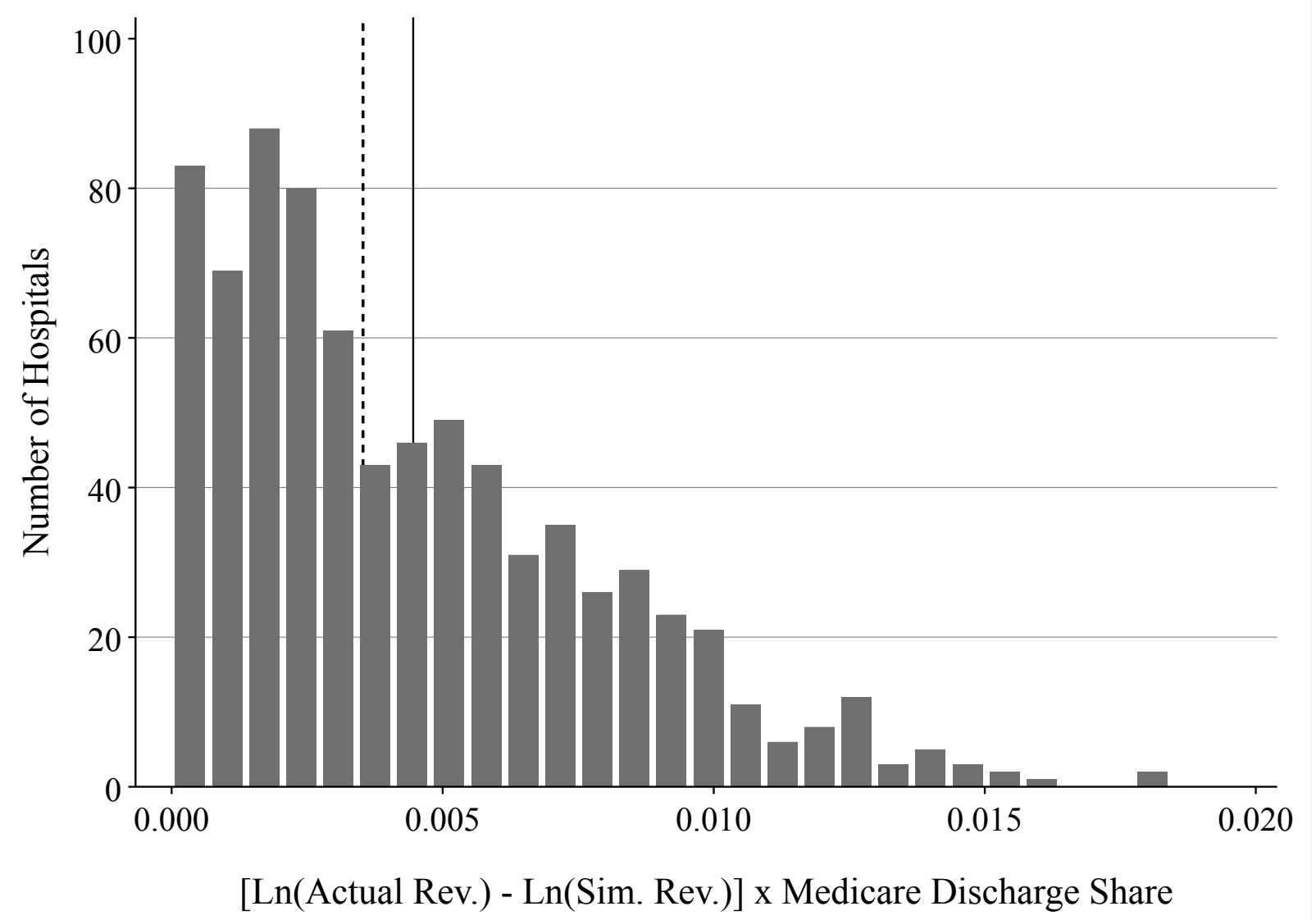

Notes: This figure shows a histogram of the BBA Bite for the teaching hospital sample, where BBA Bite is the product of (i) the difference between the (log) simulated price per discharge and actual (log) PPS price per discharge in 1995; and (ii) the Medicare share of discharges averaged over 1992-1995. The solid line indicates the mean $(0.0045)$ of this variable and the dotted lines indicates the median (0.0035). Sources: Healthcare Provider Cost Reporting Information System; Inpatient Prospective Payment System Payment Impact Files. 


\section{Figure 2: Distribution of Research Intensity}

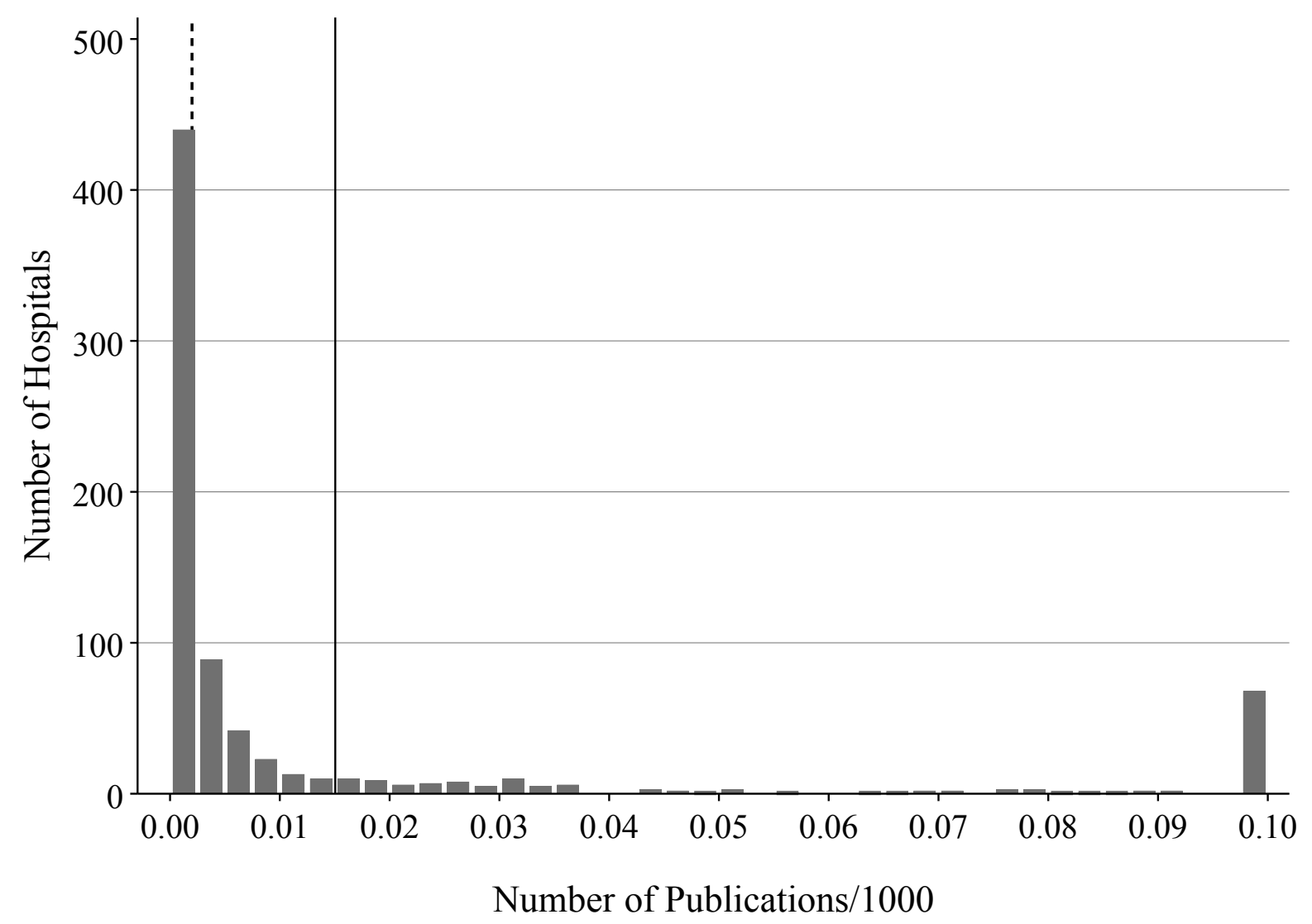

Notes: This figure shows a histogram of the Research Intensity for the teaching hospital sample, where Research Intensity is the annual number of publications averaged over 1990-1991 and divided by 1000. The solid line indicates the mean $(0.015)$ of this variable and the dotted lines indicates the median (0.002). For ease of interpretation in this figure, hospitals with Research Intensity greater than 0.1 are recoded as 0.1. Sources: Healthcare Provider Cost Reporting Information System; Inpatient Prospective Payment System Payment Impact Files. 


\section{Figure 3: Effect on the Number of Grant Applications}
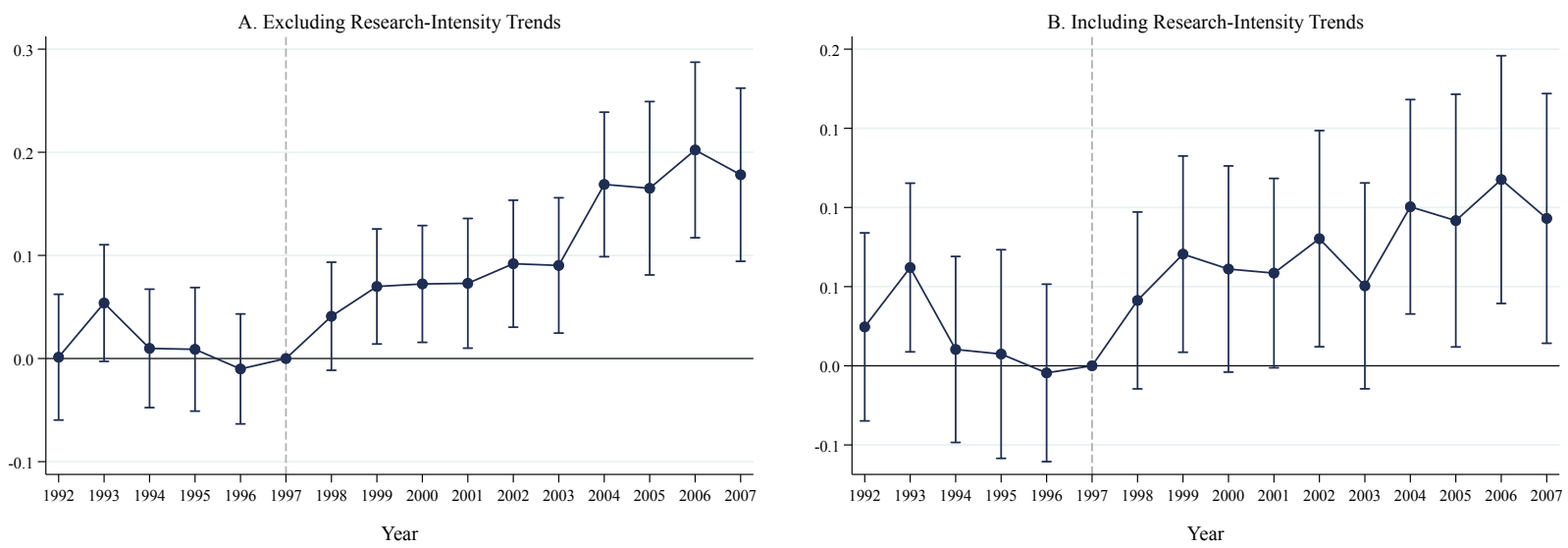

Notes: This figure plots the response in grant applications in the teaching hospital sample. Each dot corresponds to OLS coefficient estimates stemming from the event study specification in which asinh(number of grant applications) is regressed onto year fixed effects, hospital fixed effects, as well as interaction terms between an indicator for a hospital's High BBA Bite status interacted with the number of years before/after the BBA. Panel A plots estimates from a specification that excludes indicators for a hospital's High RI status interacted with the number of years before/after the start of the NIH budget doubling. Panel B plots estimates from the specification described in equation (4) and includes indicators for a hospital's High RI status interacted with the number of years before/after the start of the NIH budget doubling. The 95\% confidence interval (corresponding to robust standard errors, clustered around hospital) around these estimates is shown. Sources: Healthcare Provider Cost Reporting Information System; Inpatient Prospective Payment System Payment Impact Files; NIH IMPAC II. 
Table 1: Summary Statistics

\begin{tabular}{|c|c|c|c|c|c|c|}
\hline & count & mean & p50 & sd & $\min$ & $\max$ \\
\hline \multicolumn{7}{|l|}{ Panel A. Hospital Characteristics } \\
\hline Discharges $(1,000 \mathrm{~s})$ & 780 & 16.82 & 14.91 & 10.39 & 0.34 & 62.79 \\
\hline Inpatient Days $(1,000 \mathrm{~s})$ & 780 & 89.84 & 76.03 & 60.25 & 0.96 & 439.06 \\
\hline Medicare Teaching Payment $(\$ 1,000,000 \mathrm{~s})$ & 780 & 5.45 & 2.26 & 7.91 & 0.00 & 59.81 \\
\hline Medicare Disproportionate Share Payment $(\$ 1,000,000$ s) & 780 & 3.97 & 2.38 & 4.54 & 0.00 & 36.21 \\
\hline Total Revenue $(\$ 1,000,000 \mathrm{~s})$ & 765 & 486.94 & 374.24 & 410.95 & 14.43 & $3,182.46$ \\
\hline Beds & 780 & 360.54 & 319.41 & 202.23 & 29.12 & $1,397.62$ \\
\hline Residents and Interns & 767 & 101.92 & 41.82 & 139.35 & 0.06 & $1,097.72$ \\
\hline Pre-1992 Research (Number of Publications) & 780 & 30.34 & 2.00 & 93.50 & 0.00 & 869.50 \\
\hline BBA Bite (x100) & 780 & 0.45 & 0.35 & 0.35 & 0.00 & 1.84 \\
\hline Medicare Share of Discharges in 1995 & 780 & 0.34 & 0.34 & 0.13 & 0.02 & 0.71 \\
\hline Medicare Share of Inpatient Days in 1995 & 780 & 0.43 & 0.46 & 0.15 & 0.03 & 0.79 \\
\hline Medicare Price Per Discharge in 1995 (\$1,000s) & 780 & 8.30 & 7.48 & 2.85 & 3.67 & 27.48 \\
\hline \multicolumn{7}{|l|}{ Panel B. Grants } \\
\hline \multicolumn{7}{|l|}{ Number of Grant Applications } \\
\hline Total & 780 & 8.82 & 0.00 & 32.97 & 0.00 & 444.00 \\
\hline New & 780 & 7.15 & 0.00 & 26.43 & 0.00 & 355.75 \\
\hline Competitive Renewal & 780 & 1.67 & 0.00 & 6.60 & 0.00 & 88.25 \\
\hline Novice Investigator & 780 & 4.55 & 0.00 & 17.07 & 0.00 & 208.81 \\
\hline Seasoned Investigator & 780 & 4.10 & 0.00 & 15.44 & 0.00 & 227.25 \\
\hline MD Principal Investigator & 780 & 3.08 & 0.00 & 11.87 & 0.00 & 158.62 \\
\hline PhD Principal Investigator & 780 & 4.26 & 0.00 & 16.22 & 0.00 & 193.38 \\
\hline MD-PhD Principal Investigator & 780 & 1.36 & 0.00 & 5.81 & 0.00 & 87.75 \\
\hline \multicolumn{7}{|l|}{ Number of Grants Funded } \\
\hline Total & 780 & 2.55 & 0.00 & 10.42 & 0.00 & 147.75 \\
\hline New & 780 & 1.82 & 0.00 & 7.40 & 0.00 & 106.38 \\
\hline Competitive Renewal & 780 & 0.73 & 0.00 & 3.05 & 0.00 & 41.38 \\
\hline Novice Investigator & 780 & 1.40 & 0.00 & 5.74 & 0.00 & 74.06 \\
\hline Seasoned Investigator & 780 & 1.11 & 0.00 & 4.62 & 0.00 & 72.44 \\
\hline MD Principal Investigator & 780 & 0.96 & 0.00 & 4.05 & 0.00 & 55.00 \\
\hline PhD Principal Investigator & 780 & 1.16 & 0.00 & 4.77 & 0.00 & 61.00 \\
\hline MD-PhD Principal Investigator & 780 & 0.40 & 0.00 & 1.92 & 0.00 & 30.88 \\
\hline \multicolumn{7}{|l|}{ Panel C. Publications } \\
\hline Total & 780 & 45.40 & 2.06 & 148.55 & 0.00 & $1,683.62$ \\
\hline Citation Ranking: $\leq 25$ & 780 & 11.19 & 0.81 & 31.02 & 0.00 & 306.12 \\
\hline Citation Ranking: 26-50 & 780 & 10.41 & 0.44 & 32.00 & 0.00 & 333.38 \\
\hline Citation Ranking: 51-75 & 780 & 11.03 & 0.38 & 37.19 & 0.00 & 413.19 \\
\hline Citation Ranking: 76-95 & 780 & 9.84 & 0.25 & 37.25 & 0.00 & 456.62 \\
\hline Citation Ranking: 96-99 & 780 & 2.27 & 0.06 & 9.98 & 0.00 & 130.56 \\
\hline Citation Ranking: >99 & 780 & 0.67 & 0.00 & 3.18 & 0.00 & 45.12 \\
\hline Cited In a Patent & 780 & 11.34 & 0.25 & 43.40 & 0.00 & 547.31 \\
\hline Not Cited In a Patent & 780 & 34.07 & 1.75 & 106.05 & 0.00 & $1,136.31$ \\
\hline Disruptive & 780 & 1.69 & 0.12 & 4.86 & 0.00 & 51.00 \\
\hline Consolidating & 780 & 40.72 & 1.75 & 135.60 & 0.00 & $1,551.44$ \\
\hline Laboratory Research: Pub. with Bench MeSH & 780 & 12.41 & 0.06 & 47.18 & 0.00 & 487.50 \\
\hline Translational Research: Pub. with Translational MeSH & 780 & 12.34 & 0.25 & 42.86 & 0.00 & 516.00 \\
\hline Builds on Translational MeSH & 780 & 3.83 & 0.25 & 11.94 & 0.00 & 122.25 \\
\hline Inspires Translational MeSH & 780 & 6.40 & 0.12 & 23.48 & 0.00 & 297.81 \\
\hline Clinical Research: Clinical Trial Pub & 780 & 5.43 & 0.38 & 16.94 & 0.00 & 179.69 \\
\hline Clinical Research: Pub. with Clinical MeSH & 780 & 11.54 & 0.94 & 34.80 & 0.00 & 402.31 \\
\hline \multicolumn{7}{|c|}{ Panel D. Clinical Outcomes (Risk-Adjusted Survival Rates [30 Days]) } \\
\hline Heart Attack & 700 & 0.90 & 0.91 & 0.03 & 0.73 & 1.00 \\
\hline Heart failure & 700 & 0.96 & 0.96 & 0.02 & 0.89 & 1.01 \\
\hline Pneumonia & 700 & 0.98 & 0.98 & 0.02 & 0.89 & 1.04 \\
\hline Hip/knee & 700 & 0.95 & 0.95 & 0.02 & 0.86 & 1.04 \\
\hline
\end{tabular}

Notes: This table shows hospital characteristics for the hospitals in the teaching hospital sample between 1992-2007. All variables are measured yearly. For example, "Discharges (1000s)" is the average number of patients a hospital receives in a year. "Medicare Teaching Payment," "Medicare Disproportionate Share Payment," and "Total Revenue" are adjusted for inflation using the CPI and are measured in 1997 dollars. All hospital characteristics, grant, and publication variables have 780 observations, except for "Total Revenue" and "Residents and Interns" because of missing char in the teaching hospital sample that matched to the clinical outcomes data. Clinical outcomes are measured in three-year bins-e.g., hospital-level survival rates in 2005 are estimated over patient claims in 2003, 2004, and 2005. For each hospital, we use three-year bins for four years (1996, 2002, 2005, and 2008). Each publication's disruptiveness is measured by using the Funk and Owen-Smith (2017) disruptiveness index, denoted by $d$. "Disruptive" publications refer to publications with $d \leq 95^{t h}$ percentile and "Consolidating" publications refers to publications with $d>95^{t h}$ percentile. See Section III for detailed data descriptions. Sources: Azoulay, Greenblatt, and Heggeness (2021); Chandra et al. (2016); Funk and Owen-Smith (2017); Healthcare Provider Cost Reporting Information System; Inpatient Prospective Payment System Payment Impact Files; NIH IMPAC II; Marx and Fuegi (2020); PubMed; Web of Science. 
Table 2: Effect of BBA and NIH Budget Doubling

\begin{tabular}{|c|c|c|c|c|c|c|}
\hline & \multicolumn{2}{|c|}{ Grant Applications } & \multicolumn{2}{|c|}{ Grants Funded } & \multicolumn{2}{|c|}{ Publications } \\
\hline & (1) & (2) & (3) & (4) & $(5)$ & (6) \\
\hline BBA Bite $\times$ Post BBA & $\begin{array}{c}19.065^{* * *} \\
(4.284)\end{array}$ & $\begin{array}{l}9.338^{* *} \\
(4.497)\end{array}$ & $\begin{array}{c}15.545^{* * *} \\
(3.325)\end{array}$ & $\begin{array}{l}7.170^{* *} \\
(3.430)\end{array}$ & $\begin{array}{c}6.148 \\
(5.380)\end{array}$ & $\begin{array}{c}1.510 \\
(5.893)\end{array}$ \\
\hline RI $\times$ Post NIH Doubling & & $\begin{array}{c}1.009^{* * *} \\
(0.207)\end{array}$ & & $\begin{array}{c}0.869^{* * *} \\
(0.209)\end{array}$ & & $\begin{array}{c}0.481^{* * *} \\
(0.120)\end{array}$ \\
\hline Elasticity (BBA Bite) & 0.053 & 0.026 & 0.047 & 0.021 & 0.017 & 0.004 \\
\hline Elasti & & 0.017 & & 0.016 & & 0.008 \\
\hline Adjusted $R^{2}$ & 0.023 & 0.039 & 0.018 & 0.038 & 0.029 & 0.030 \\
\hline Mean of Outcome & 0.751 & 0.751 & 0.439 & 0.439 & 1.991 & 1.991 \\
\hline Nb. Observations & 12,480 & 12,480 & 12,480 & 12,480 & 12,480 & 12,480 \\
\hline Nb. Hospitals & 780 & 780 & 780 & 780 & 780 & 780 \\
\hline
\end{tabular}

Notes: This table reports difference-in-differences estimates of the effect of the BBA and NIH budget doubling on the number of grant applications, grants funded, and publications, in the teaching hospital sample. Observations are at the hospital-year level. Outcomes are transformed with the inverse hyperbolic sine function. BBA Bite is a hospital's BBA Bite and RI is a hospital's research intensity (average number of annual publications between 1990-1991 divided by 1000). Estimates are from OLS regressions, and each coefficient is from a separate regression. Robust standard errors (clustered at the hospital level) are in parentheses. Sources: Healthcare Provider Cost Reporting Information System; Inpatient Prospective Payment System Payment Impact Files; NIH IMPAC II; PubMed, Web of Science.

${ }^{*} p<0.10,{ }^{* *} p<0.05,{ }^{* * *} p<0.001$. 
Table 3: Effect on the Number of Grant Applications

\begin{tabular}{|c|c|c|c|c|c|c|c|c|}
\hline & \multirow[b]{2}{*}{$\begin{array}{l}\text { Total } \\
(1)\end{array}$} & \multicolumn{2}{|c|}{ Grant Cycle } & \multicolumn{2}{|c|}{$\begin{array}{c}\text { Principal Investigator } \\
\text { Experience }\end{array}$} & \multicolumn{3}{|c|}{$\begin{array}{c}\text { Principal Investigator } \\
\text { Degree }\end{array}$} \\
\hline & & $\begin{array}{l}\text { New } \\
(2)\end{array}$ & $\begin{array}{c}\text { Renewal } \\
(3)\end{array}$ & $\begin{array}{l}\text { Novice } \\
(4)\end{array}$ & $\begin{array}{c}\text { Incumbent } \\
(5)\end{array}$ & $\begin{array}{l}\mathrm{MD} \\
(6)\end{array}$ & $\begin{array}{c}\mathrm{PhD} \\
(7)\end{array}$ & $\begin{array}{c}\mathrm{MD}-\mathrm{PhD} \\
(8)\end{array}$ \\
\hline BBA Bite $\times$ Post BBA & $\begin{array}{l}9.338^{* *} \\
(4.497)\end{array}$ & $\begin{array}{l}13.13^{* *} \\
(4.604)\end{array}$ & $\begin{array}{l}-0.505 \\
(2.841)\end{array}$ & $\begin{array}{c}27.16^{* * *} \\
(4.482)\end{array}$ & $\begin{array}{l}3.665 \\
(4.065)\end{array}$ & $\begin{array}{l}8.686^{* *} \\
(4.424)\end{array}$ & $\begin{array}{l}11.79^{* *} \\
(4.178)\end{array}$ & $\begin{array}{c}14.60^{* * *} \\
(3.789)\end{array}$ \\
\hline Elasticity & 0.026 & 0.037 & -0.002 & 0.077 & 0.011 & 0.025 & 0.034 & 0.050 \\
\hline Adjusted $R^{2}$ & 0.039 & 0.057 & 0.010 & 0.134 & 0.024 & 0.033 & 0.068 & 0.101 \\
\hline Diff. Wald test p-value & & \multicolumn{2}{|c|}{0.001} & \multicolumn{2}{|c|}{0.000} & & 0.509 & 0.237 \\
\hline Mean of Outcome & 0.751 & 0.705 & 0.372 & 0.541 & 0.587 & 0.519 & 0.533 & 0.328 \\
\hline Nb. Observations & 12,480 & 12,480 & 12,480 & 12,480 & 12,480 & 12,480 & 12,480 & 12,480 \\
\hline Nb. Hospitals & 780 & 780 & 780 & 780 & 780 & 780 & 780 & 780 \\
\hline
\end{tabular}

Notes: This table reports difference-in-differences estimates of the effect of the BBA on the number of grant applications, in the teaching hospital sample. Observations are at the hospital-year level. Outcomes are transformed with the inverse hyperbolic sine function. Column 1 presents results from OLS regressions. As shown in equation (3), regressions include hospital fixed effects, year fixed effects, and RI $\times$ Post NIH Doubling, where RI is the average number of publications between 1990-1991 divided by 1000. Columns 2-8 present estimates from seemingly unrelated regressions. Each coefficient is from a separate regression. The elasticity of 0.026 implies that a one percentage increase in BBA Bite is associated on average with a 0.026 percent yearly increase in grant applications following the BBA's enactment. The fifth row shows $p$-values from Wald tests that compare coefficients on BBA Bite $\times$ Post BBA across different columns (Column 2 vs. Column 3; Column 4 vs. Column 5; Column 6 vs. Column 7; Column 6 vs. Column 8). Robust standard errors (clustered at the hospital level) are in parentheses. Sources: Healthcare Provider Cost Reporting Information System; Inpatient Prospective Payment System Payment Impact Files; NIH IMPAC II.

${ }^{*} p<0.10,{ }^{* *} p<0.05,{ }^{* * *} p<0.001$. 


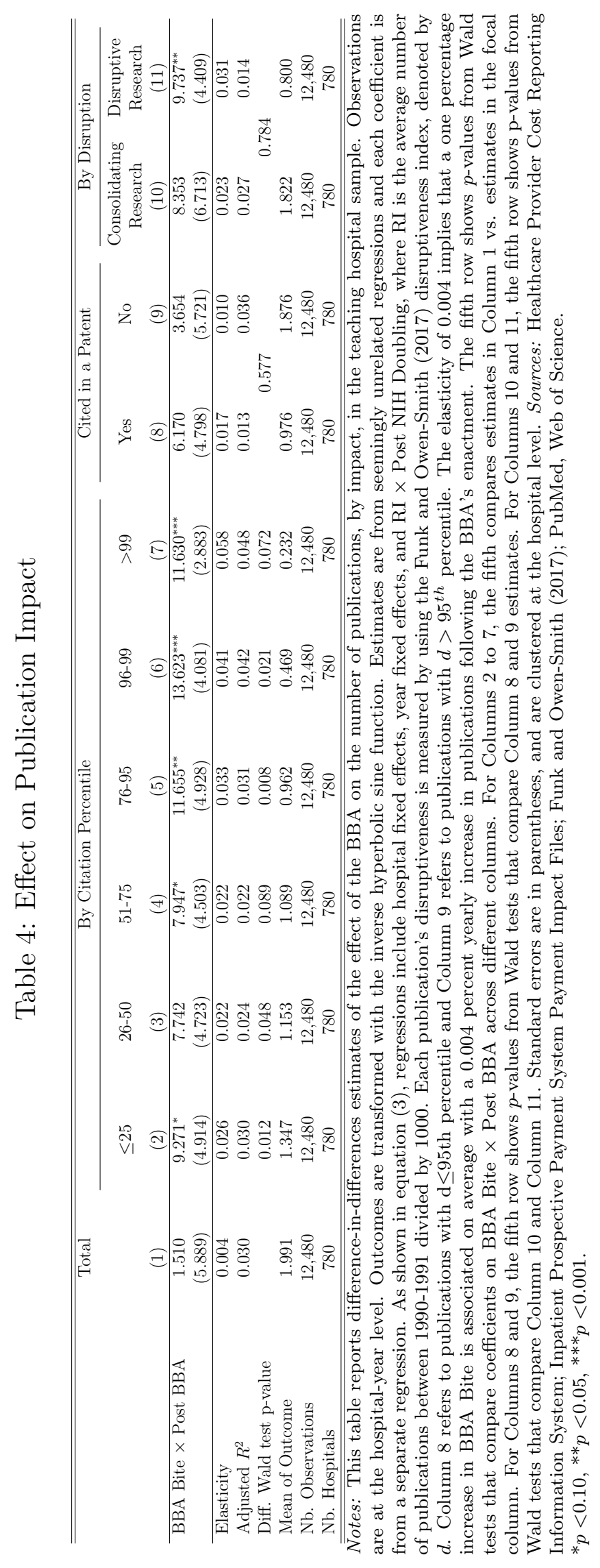


Table 5: Effect on Publication Composition

\begin{tabular}{|c|c|c|c|c|c|c|}
\hline & \multirow{2}{*}{$\begin{array}{c}\begin{array}{c}\text { Laboratory } \\
\text { Research }\end{array} \\
\text { Bench } \\
\text { MeSH }\end{array}$} & \multicolumn{3}{|c|}{ Translational Research } & \multicolumn{2}{|c|}{ Clinical Research } \\
\hline & & $\begin{array}{c}\text { Translational } \\
\text { MeSH } \\
(2)\end{array}$ & $\begin{array}{c}\text { Builds on } \\
\text { Translational } \\
\text { MeSH } \\
(3)\end{array}$ & $\begin{array}{c}\text { Inspiring } \\
\text { Translational } \\
\text { MeSH } \\
(4)\end{array}$ & $\begin{array}{l}\text { Clinical Trials } \\
\qquad(5)\end{array}$ & $\begin{array}{l}\text { Other } \\
(6)\end{array}$ \\
\hline BBA Bite $\times$ Post BBA & $\begin{array}{l}-6.146 \\
(5.206)\end{array}$ & $\begin{array}{l}8.618^{*} \\
(4.755)\end{array}$ & $\begin{array}{c}22.493^{* * *} \\
(4.357)\end{array}$ & $\begin{array}{c}13.859^{* *} \\
(4.466)\end{array}$ & $\begin{array}{c}24.496^{* * *} \\
(4.650)\end{array}$ & $\begin{array}{c}10.560^{* *} \\
(5.087)\end{array}$ \\
\hline Elasticity & -0.017 & 0.024 & 0.065 & 0.039 & 0.069 & 0.030 \\
\hline Adjusted $R^{2}$ & 0.001 & 0.020 & 0.080 & 0.032 & 0.098 & 0.027 \\
\hline Mean of Outcome & 0.826 & 1.062 & 0.798 & 0.804 & 0.950 & 1.398 \\
\hline Nb. Observations & 12,480 & 12,480 & 12,480 & 12,480 & 12,480 & 12,480 \\
\hline Nb. Hospitals & 780 & 780 & 780 & 780 & 780 & 780 \\
\hline
\end{tabular}

Notes: This table reports difference-in-differences estimates of the effect of the BBA on bench, translational, and clinical trial research in hospitals, in the teaching hospital sample. Observations are at the hospital-year level. Outcomes are transformed with the inverse hyperbolic sine function. Estimates are from OLS regressions and each coefficient is from a separate regression. As shown in equation (3), regressions include hospital fixed effects, year fixed effects, and RI $\times$ Post NIH Doubling, where RI is the average number of publications between 1990-1991 divided by 1000. Column 1 refers to publications that are not disease-oriented, are not clinical trial publications, and rely on either a molecular biology technique, a model organism, cellular structures and macromolecules, or biochemical and cellular processes (based on MeSH terms). Column 2 refers to publications that are disease-oriented, are not clinical trial publications, and rely on either a molecular biology technique, a model organism, cellular structures and macromolecules, or biochemical and cellular processes (based on MeSH terms). Column 3 refers to publications that report the results of a clinical trials, or are tagged by a human MeSH term and also cite a translational publication. Column 4 refers to publications that are translational and is cited by a clinical trial publication (or one that contains a human MeSH term). Column 5 refers to publications that are indicated as clinical trials based on MeSH terms or the publication type field in PubMed. Finally, Column 6 refers to publications that are disease-oriented, not clinical trials, and not tagged by any bench MeSH keywords. The elasticity of -0.017 implies that a one percentage increase in BBA Bite is associated on average with a 0.017 percent yearly decrease in publications following the BBA's enactment. Standard errors are in parentheses, and are clustered at the hospital level. Sources: Healthcare Provider Cost Reporting Information System; Inpatient Prospective Payment System Payment Impact Files; Azoulay, Greenblatt, and Heggeness (2021); Marx and Fuegi (2020); PubMed; Web of Science.

${ }^{*} p<0.10,{ }^{* *} p<0.05,{ }^{* * *} p<0.001$. 
Table 6: Effect on Clinical Outcomes (Changes in Risk-adjusted Survival Rates)

\begin{tabular}{lcccc}
\hline \hline & Heart Attack & Heart Failure & Hip/Knee & Pneumonia \\
& $(1)$ & $(2)$ & $(3)$ & $(4)$ \\
\hline BBA Bite & -0.4286 & -0.4028 & 0.4099 & -0.0743 \\
& $(0.4227)$ & $(0.2537)$ & $(0.2783)$ & $(0.3146)$ \\
Ln(Discharges in 1995) & 0.0016 & 0.0018 & -0.0003 & $0.0046^{* *}$ \\
& $(0.0025)$ & $(0.0015)$ & $(0.0014)$ & $(0.0019)$ \\
Adjusted $R^{2}$ & -0.0012 & 0.0016 & 0.0004 & 0.0076 \\
\hline Mean of Outcome & 0.0270 & 0.0106 & -0.0005 & 0.0147 \\
Nb. Observations & 700 & 700 & 700 & 700 \\
\hline \hline
\end{tabular}

Notes: This table displays the effect on changes in risk-adjusted survival rates among hospitals in the teaching hospital sample. The hospital sample used is the subset of the teaching hospital sample that is matched to the clinical outcomes dataset from Chandra et al. (2016). Observations are at the hospital-level. Outcomes are the difference in average survival rates between the post-BBA time period and the pre-BBA time period. Estimates are from OLS regressions and each coefficient is from a separate regression. Standard errors are in parentheses and robust. Sources: Healthcare Provider Cost Reporting Information System; Inpatient Prospective Payment System Payment Impact Files; Chandra et al. (2016).

${ }^{*} p<0.10,{ }^{* *} p<0.05,{ }^{* * *} p<0.001$. 


\section{Appendix A: Medicare Inpatient Reimbursement}

Overview of Medicare Payments. This section provides an overview of how Medicare reimburses hospitals for care provided to beneficiaries, which will be useful to understand the impact of the reform analyzed in this paper.

Since 1984, payments have been under the Prospective Payment System (PPS). Under the PPS, hospitals receive a fixed payment for a given medical diagnosis (called a "Diagnosis Related Group (DRG)"), regardless of hospital expenditures. Each DRG payment consists of three components: (i) a DRG weight, (ii) a base payment, and (iii) adjustments. Put differently, the PPS payment received by hospital $h$ for an admission in DRG $d$ in year $t$ is given by:

$$
P P S_{h, d, t}=f\left(D R G_{d, t}, p_{h, t}, \text { adjustments }_{h, t}\right)
$$

where $D R G_{d, t}$ is a weight and reflects hospitals' aggregate historical costs of treating patients in DRG $d$. The average DRG weight in a hospital, or the Medicare case-mix, reflects the severity of treatment for the average patient. The base payment $\left(p_{h, t}\right)$ is a factor that converts the DRG weight to dollars. The base payment is updated annually and is set nationally, but is adjusted based on local market conditions, such as prices for labor. Finally, three types of adjustments are provided: indirect medical education (IME) subsidies are payments received by hospitals for training physicians, disproportionate share hospital (DSH) payments are additional payments for treating poor patients, and outlier payments are made to compensate providers for patients with exceptionally costly stays (Keeler, Carter, and Trude 1988).

The BBA changed the formula for these three adjustments. In this paper, our payment calculations are centered around changes to the IME and DSH payments, as data limitations do not allow us to calculate simulated outlier payments. We now describe IME and DSH payments in further detail.

Indirect Medical Education Subsidies. Teaching hospitals receive two supplemental payments from Medicare: direct medical education (DME) and indirect medical education (IME) payments. DME payments reimburse a teaching hospital for Medicare's share of the direct costs of training residents, such as salaries paid to interns, residents, and teaching physicians. The IME adjustment is a percentage add-on payment to the hospital's basic DRG payment (Fishman 1993). IME payments are meant to compensate teaching hospitals for indirect expenses stemming for example from use of diagnostic services by clinically inex- 
perienced residents or decreased productivity of nurses and support staff involved in teaching of residents. Since 1989, the DRG payment a hospital receives for admitting a Medicare patient increases non-linearly with the hospital's resident-to-bed ratio and a multiplier.

$$
\text { ime }_{h, t}=\alpha_{t} \times\left[\left(1+\frac{\text { resident }}{h, t}\right)^{.405}-1\right]
$$

where $\alpha$ is a multiplier set at 1.89 in the pre-reform period. This correspond to a price increase of approximately $7.65 \%$ for every $10 \%$ increase in a hospital's resident-to-bed ratio.

Disproportionate Share Hospital Subsidies The Medicare DSH adjustment was enacted by the Consolidated Omnibus Budget Reconciliation Act of 1985 and became effective in 1986. Like the IME adjustment, the DSH adjustment is a percentage add-on to the hospital's basic DRG payment. The key determinant of whether a hospital is eligible for this subsidy is the fraction of total patient-days allocated to poor patients. Above a certain threshold, hospitals become eligible for a DSH payment adjustment, which varies according to whether the hospital is urban or rural, is a sole community hospital, and the number of beds. ${ }^{i}$

The Balanced Budget Act. The BBA reduced both IME payments: beginning in FY1998, there were planned reductions in the IME multiplier ( $\alpha$ in Appendix A equation (2)). Similarly, the BBA reduced DSH payments through imposing an overall percentage reduction in DSH payments from FY1998 to FY2002 (e.g., the BBA reduced DSH payments by 1\% in FY1998, 2\% in FY1999, etc.). Subsequent reforms (BBRA and BIPA) somewhat dampened the negative effect of the BBA on IME and DSH payments. For a detailed explanation of how the BBA changed DRG payment adjustments, see Wu (2010).

Relationship Between Pre-BBA Research and the Simulated Revenue Per Discharge Change The bin scatter plot in Appendix Figure A1 describes how a hospital's average pre-BBA research levels relate to the simulated revenue per discharge change described in Section III.B. Each hospital's annual grant applications (Panel A), grants funded (Panel B), and publications (Panel C) are averaged over 1992-1995 and plotted against the simulated revenue per discharge change.

${ }^{\mathrm{i}}$ For more details, see https://www.cms.gov/Medicare/Medicare-Fee-for-Service-Payment/ AcuteInpatientPPS/dsh.html. 
Figure A1: Pre-BBA Research and Simulated Revenue Change
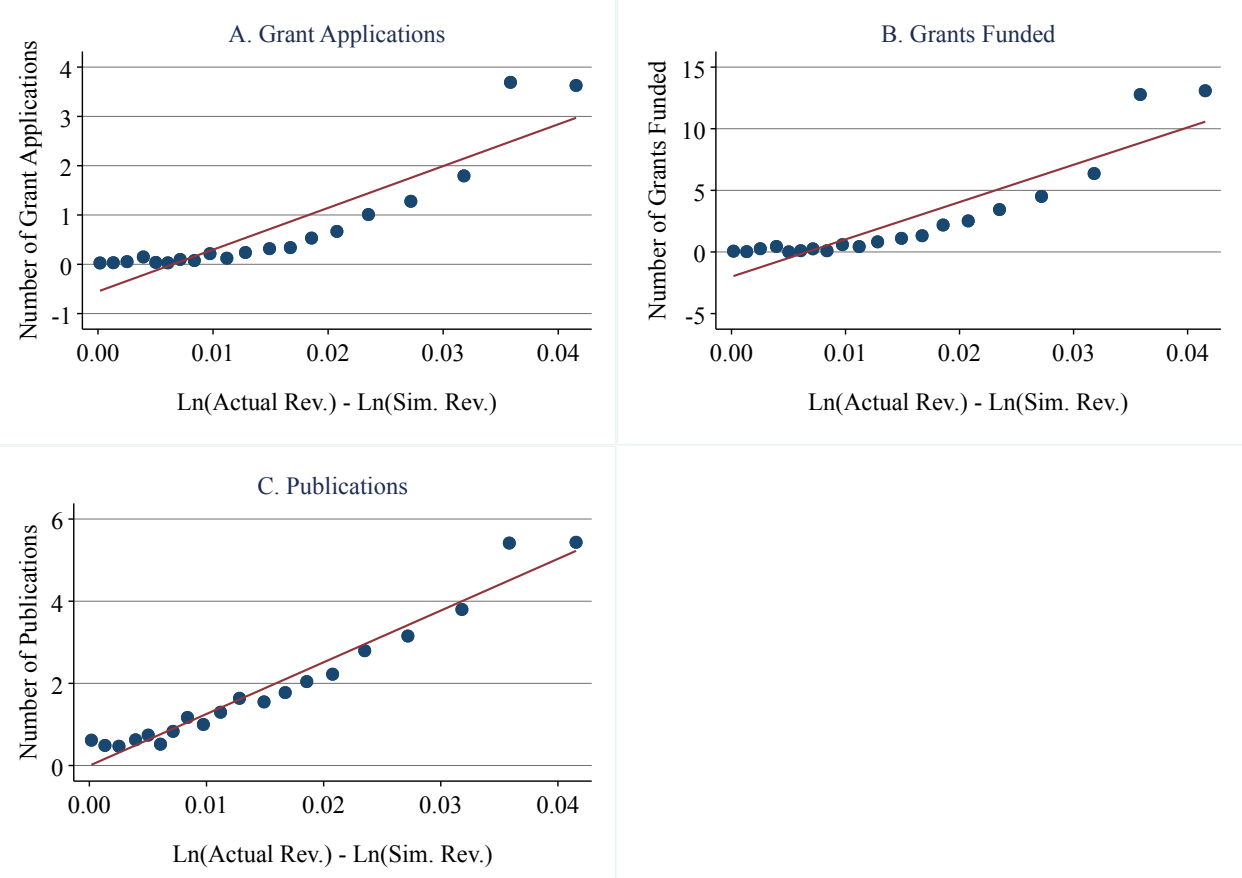

Notes: This figure plots the relationship between pre-BBA research levels (averaged over 1992-1995) and the simulated PPS revenue change. Research outcomes are transformed with the inverse hyperbolic sine function. Sources: Healthcare Provider Cost Reporting Information System; Inpatient Prospective Payment System Payment Impact Files; NIH IMPAC II; PubMed; Web of Science. 


\section{Appendix B: Extra Figures and Tables}

This Appendix contains additional figures and tables. 


\section{Figure B1: Data Sources and Variable Construction}

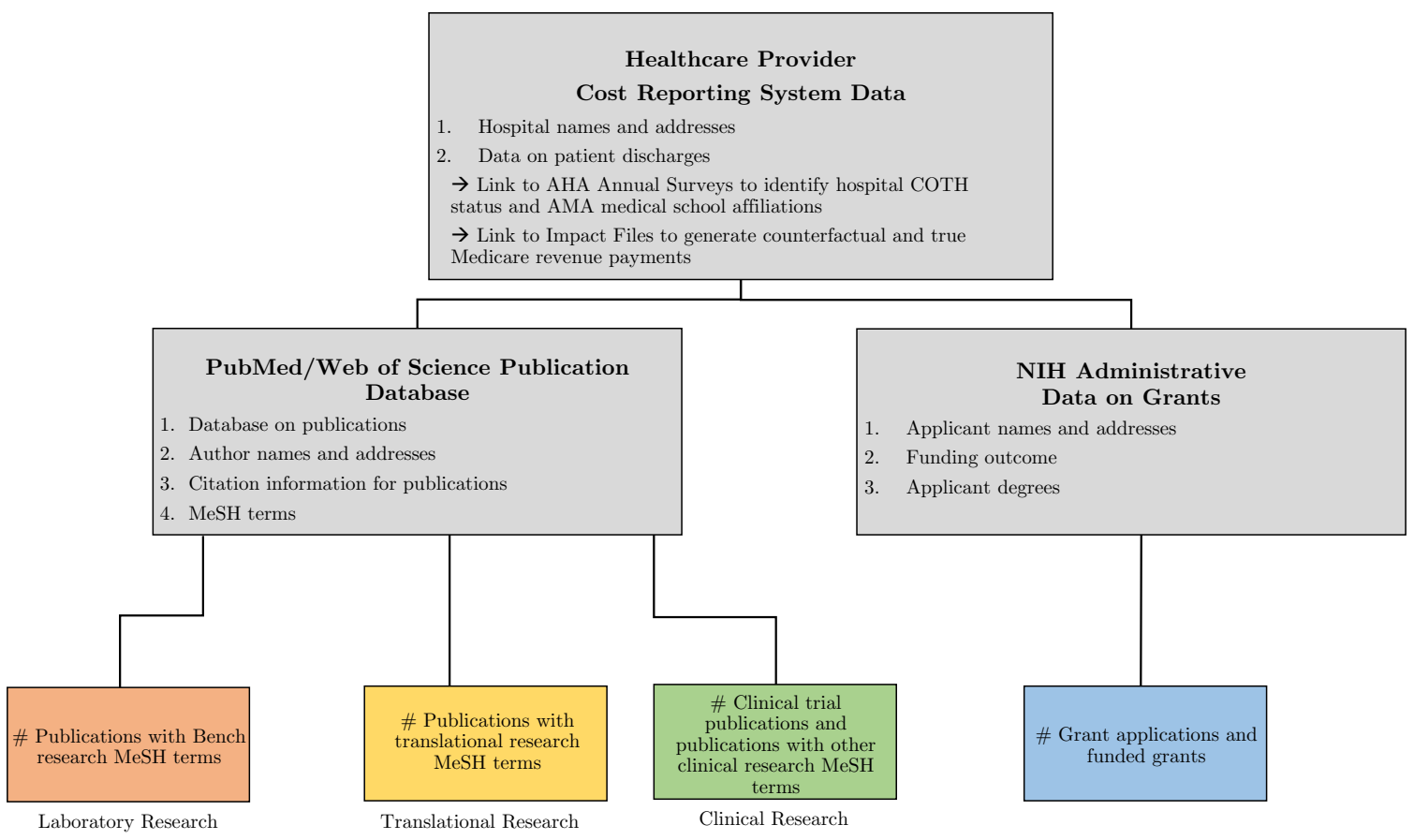




\section{Figure B2: Distribution of Research Outcomes}
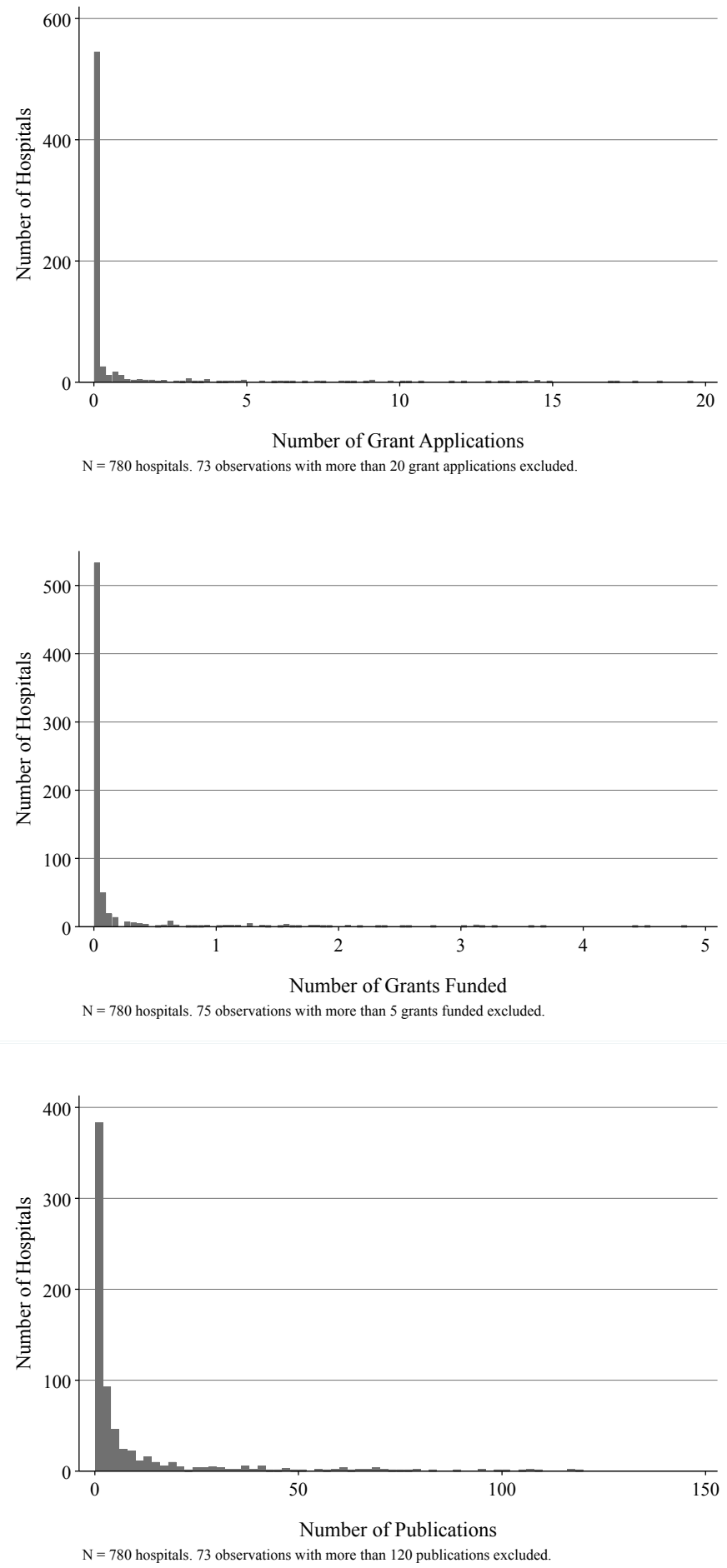

Notes: These figure shows histograms of the research outcomes across hospitals in the teaching hospital sample. Sources: NIH IMPAC II; PubMed; Web of Science. 
Figure B3: Change in NIH Grant Applications, 1992-2007

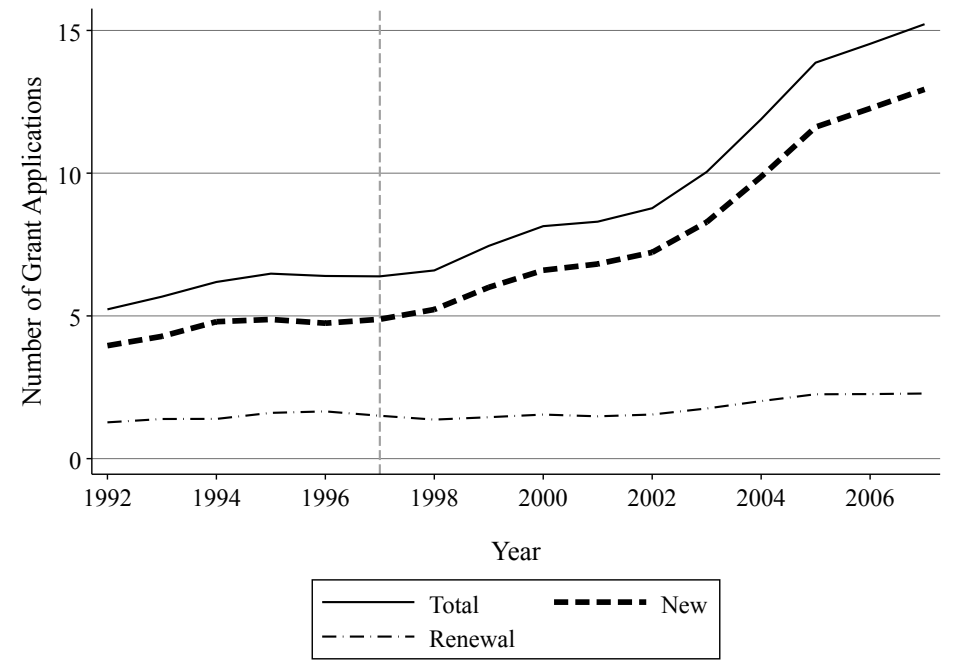

Notes: This figure plots the annual number of grant applications, averaged across all hospitals in the teaching hospital sample. The dashed line indicates the year in which the BBA came into effect. Source: NIH IMPAC II.

Figure B4: Change in NIH Grants Funded, 1992-2007

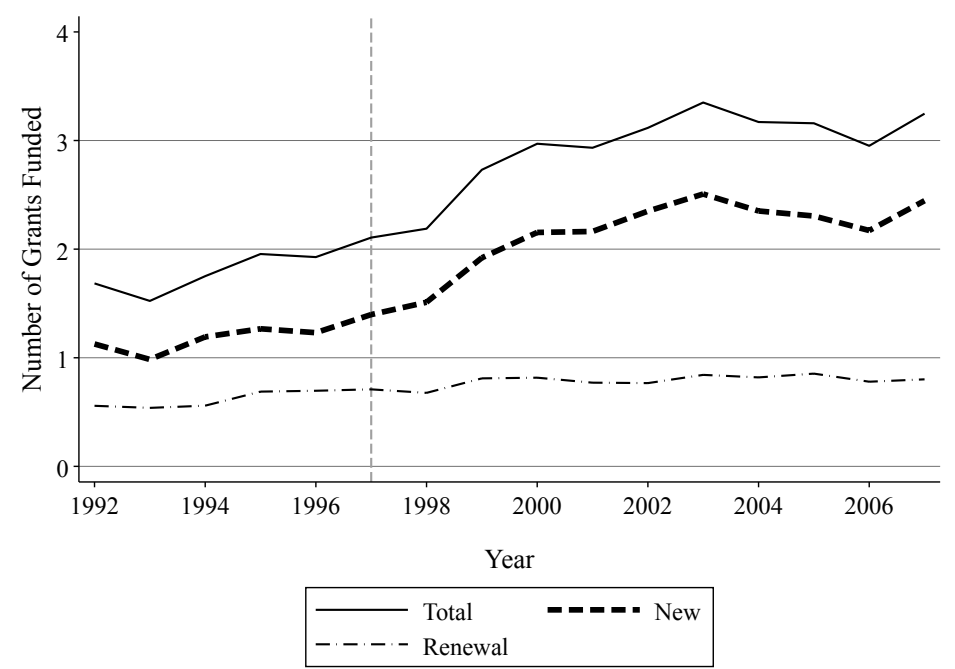

Notes: This figure plots the annual number of grants funded, averaged across all hospitals in the teaching hospital sample. The dashed line indicates the year in which the BBA came into effect. Source: NIH IMPAC II. 
Figure B5: Change in Publications, 1992-2007

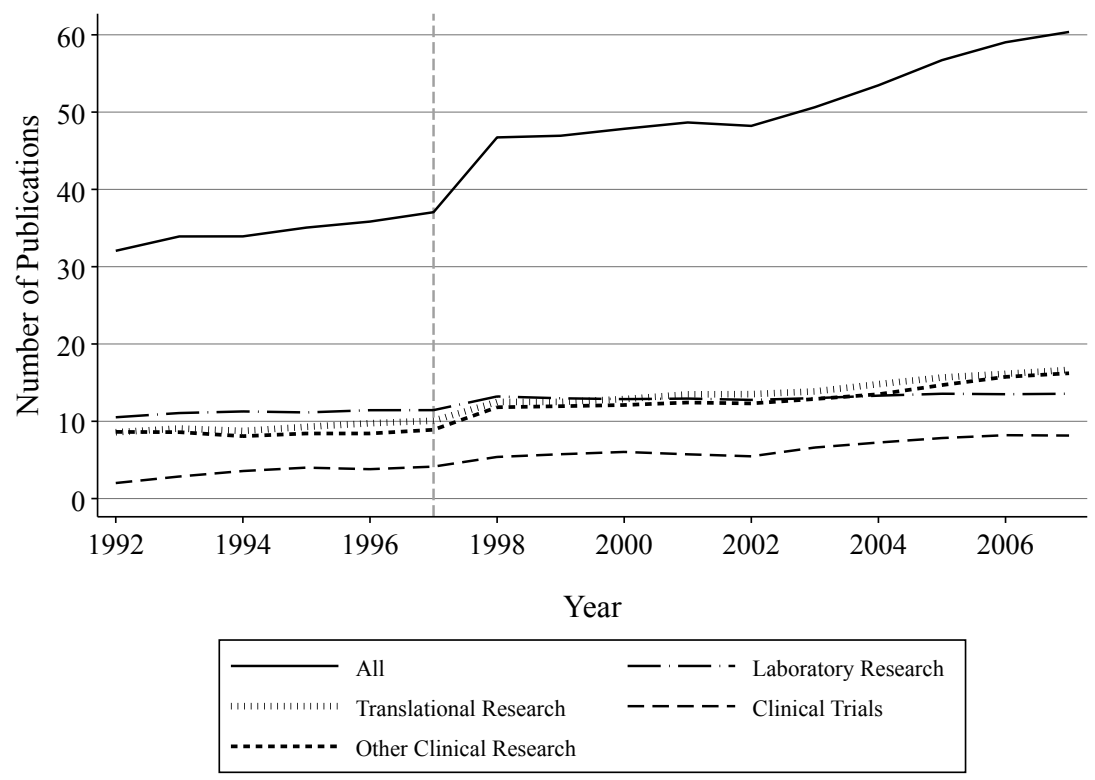

Notes: This figure plots the annual number of publications, averaged across all hospitals in the teaching hospital sample. The dashed vertical line indicates the year in which the BBA came into effect. "Laboratory Research" refers to publications that are not disease-oriented, are not clinical trial publications, and rely on either a molecular biology technique, a model organism, cellular structures and macromolecules, or biochemical and cellular processes (based on MeSH terms). "Translational Research" refers to publications that are disease-oriented, are not clinical trial publications, and rely on either a molecular biology technique, a model organism, cellular structures and macromolecules, or biochemical and cellular processes (based on MeSH terms). "Clinical Trials" refers to publications are indicated as clinical trials based on MeSH terms or the publication type field in PubMed. "Other Clinical Research" refers to publications that are disease-oriented, not clinical trials, and not tagged by any bench MeSH keywords. The discrete shift in total publications in 1998 is likely explained by our strategy for constructing the publication dataset, as discussed in Section III. Sources: Azoulay, Greenblatt, and Heggeness (2021); PubMed; Web of Science. 
Figure B6: Risk-Adjusted 30-Day Survival Rates
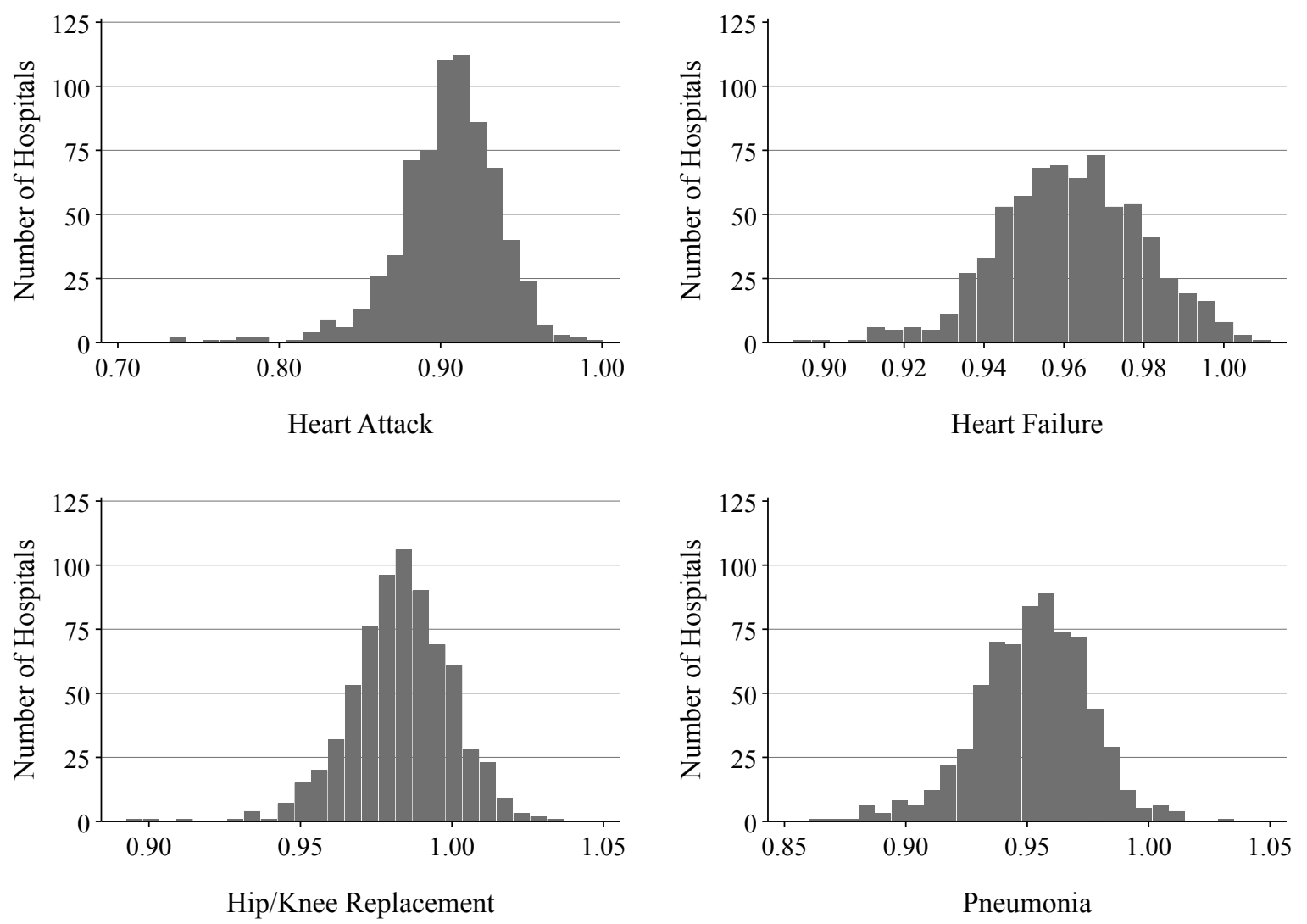

Notes: These figure shows histograms of the average risk-adjusted 30 day survival rates across hospitals in the teaching hospital sample. The clinical outcomes data characterize patient outcomes following a heart attack, heart failure, hip/knee replacement, and pneumonia. Source: Chandra et al. (2016). 
Figure B7: Hospital Research Intensity and BBA Bite

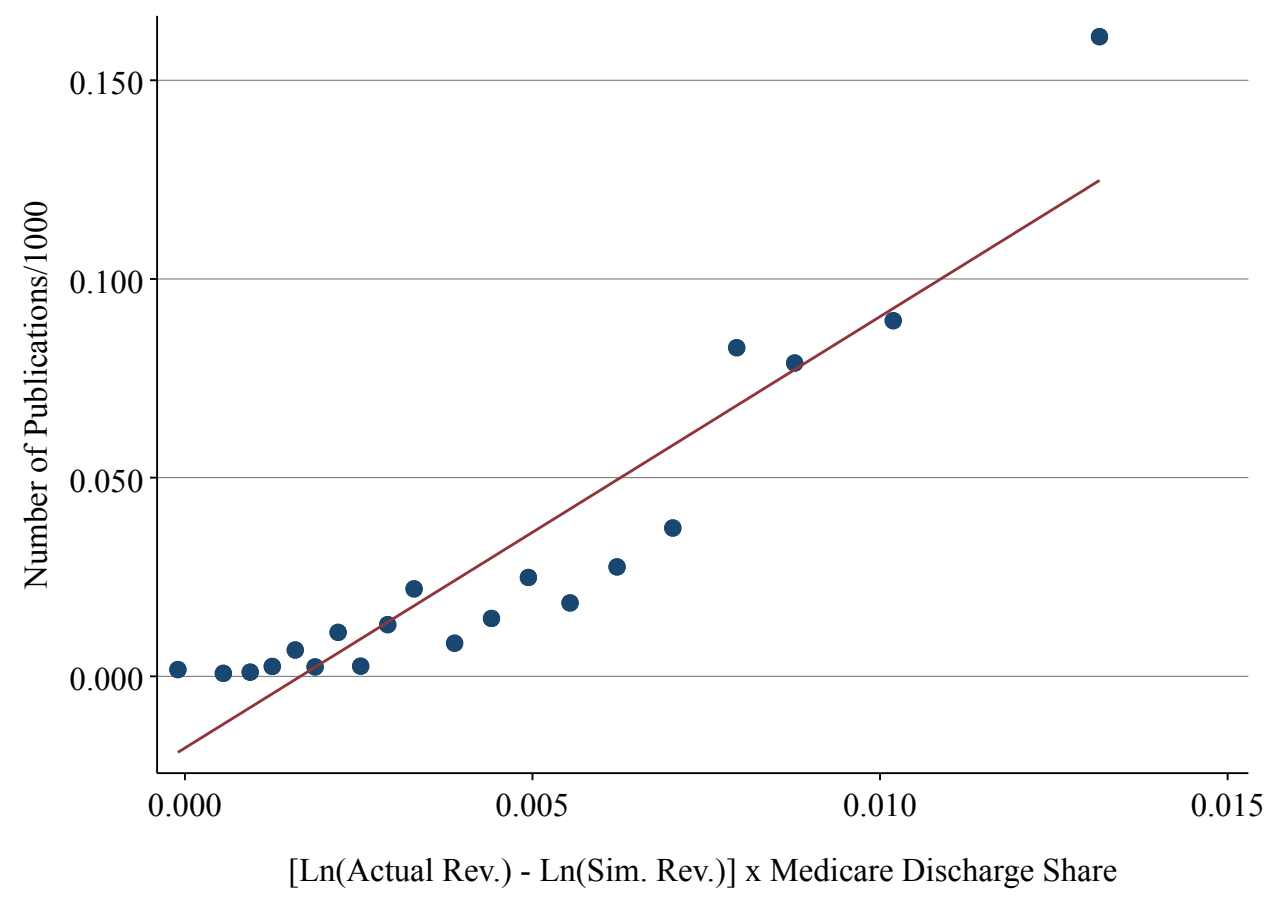

Notes: This figure plots the relationship between pre-BBA hospital research intensity (as measured by average annual publications between 1990-1991 divided by 1000) and hospital BBA Bite. Sources: Healthcare Provider Cost Reporting Information System; Inpatient Prospective Payment System Payment Impact Files; NIH IMPAC II; PubMed; Web of Science. 


\section{Figure B8: Effect on the Number of Grants Funded}
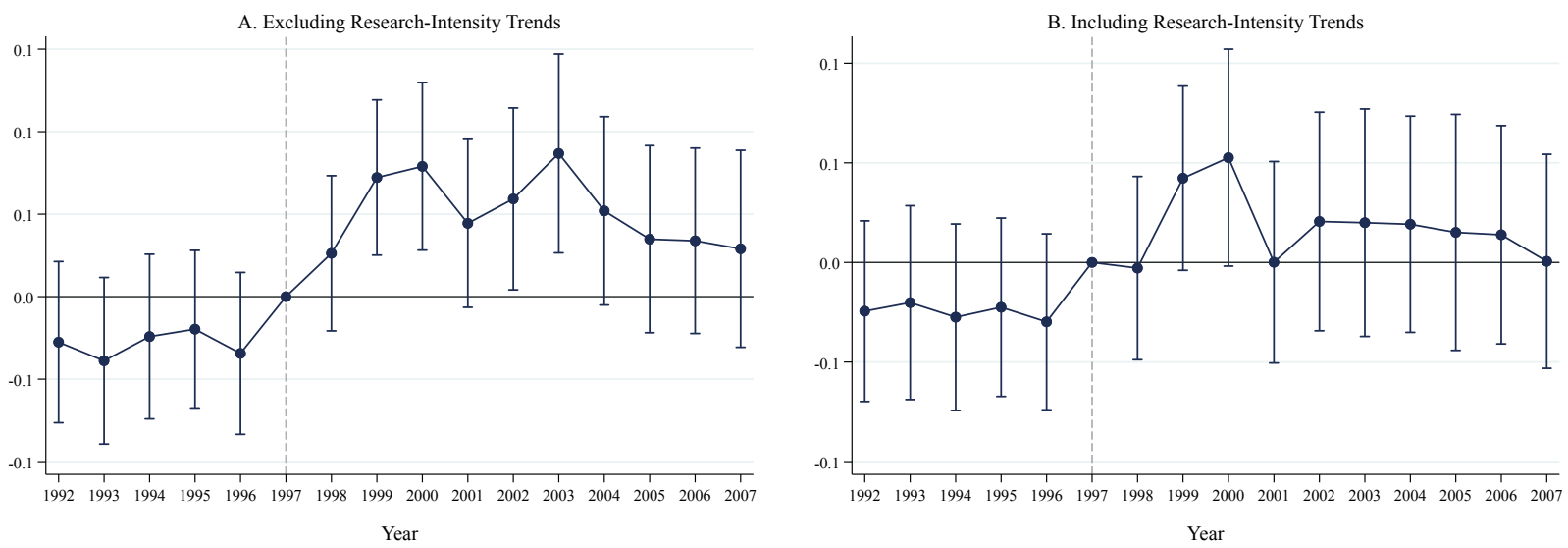

Notes: This figure plots the response in grants funded in the teaching hospital sample. Each dot corresponds to OLS coefficient estimates stemming from the event study specification described in Section IV, in which asinh(number of grants funded) is regressed onto year fixed effects, hospital fixed effects, as well as interaction terms between an indicator for a hospital's High BBA Bite status interacted with the number of years before/after the BBA. Panel A plots estimates from a specification that excludes indicators for a hospital's High RI status interacted with the number of years before/after the start of the NIH budget doubling. Panel B plots estimates from the specification described in equation (4) and includes indicators for a hospital's High RI status interacted with the number of years before/after the start of the NIH budget doubling. The $95 \%$ confidence interval (corresponding to robust standard errors, clustered around hospital) around these estimates is shown. Sources: Healthcare Provider Cost Reporting Information System; Inpatient Prospective Payment System Payment Impact Files; NIH IMPAC II. 


\section{Figure B9: Effect on the Number of Publications}
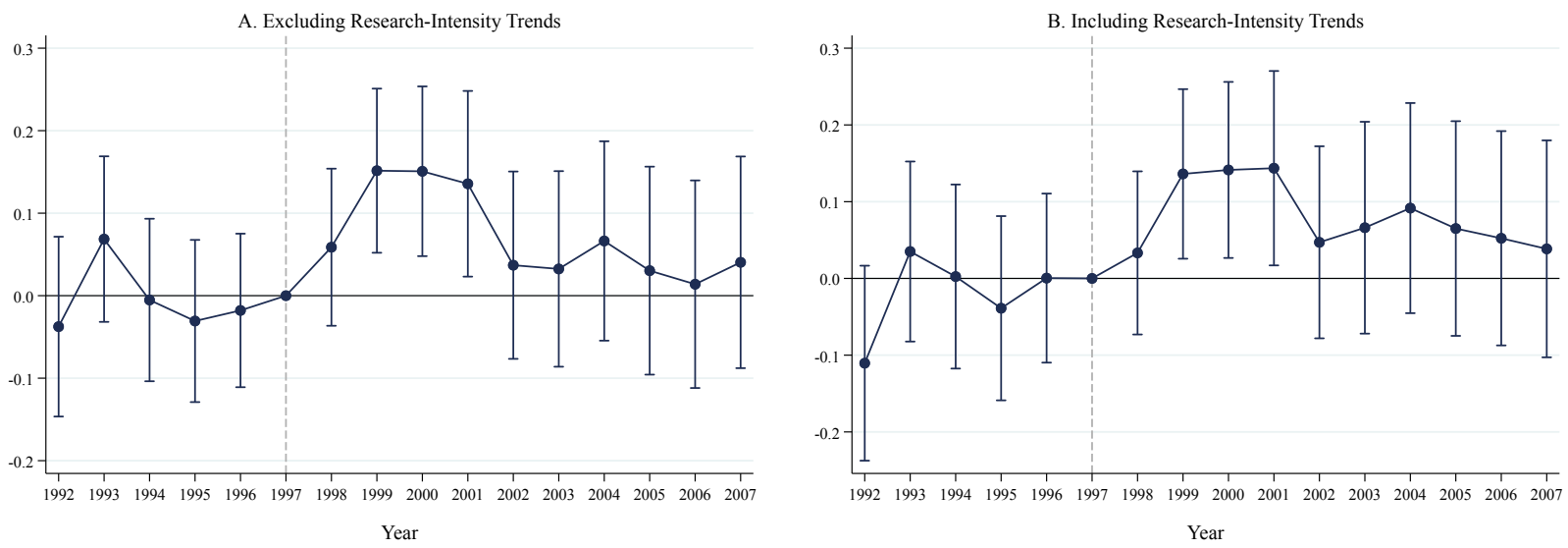

Notes: This figure plots the response in publications in the teaching hospital sample. Each dot corresponds to OLS coefficient estimates stemming from the event study specification described in Section IV, in which asinh(number of publications) is regressed onto year fixed effects, hospital fixed effects, as well as interaction terms between an indicator for a hospital's High BBA Bite status interacted with the number of years before/after the BBA. Panel A plots estimates from a specification that excludes indicators for a hospital's High RI status interacted with the number of years before/after the start of the NIH budget doubling. Panel B plots estimates from the specification described in equation (4) and includes indicators for a hospital's High RI status interacted with the number of years before/after the start of the NIH budget doubling. The 95\% confidence interval (corresponding to robust standard errors, clustered around hospital) around these estimates is shown. Sources: Healthcare Provider Cost Reporting Information System; Inpatient Prospective Payment System Payment Impact Files; PubMed, Web of Science. 
Figure B10: Effect on the Number of Grant Applications

Across Newly Hired and Seasoned Researchers

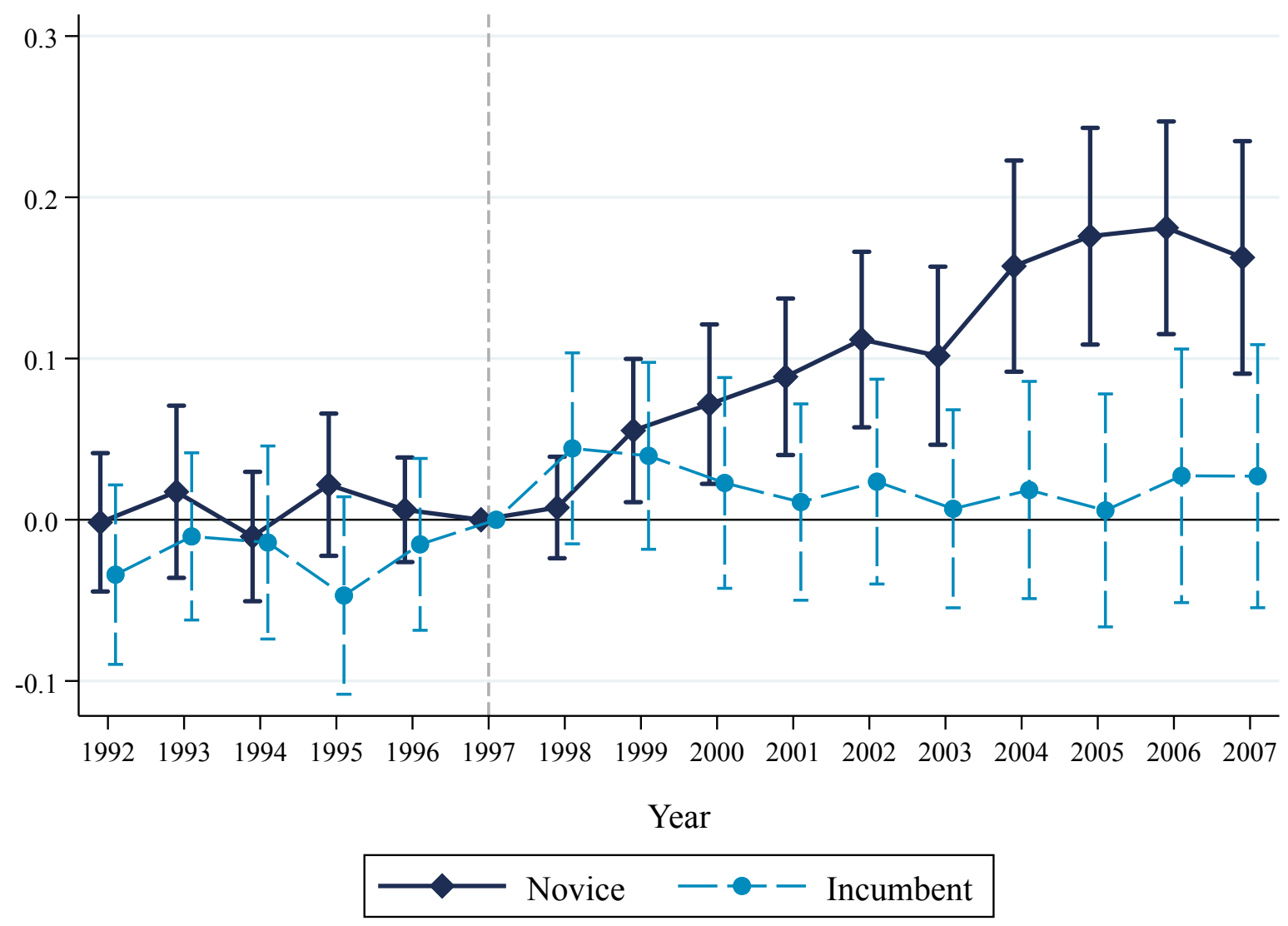

Notes: This figure plots the response in grants applications across newly hired and seasoned researchers. Each dot corresponds to OLS coefficient estimates stemming from the event study specification described in Section IV, in which asinh(number of grant applications) is regressed onto year fixed effects, hospital fixed effects, interaction terms between an indicator for a hospital's High BBA Bite status interacted with the number of years before/after the BBA, and interaction terms between an indicator for a hospital's High RI status interacted with the number of years before/after the start of the NIH budget doubling. The 95\% confidence interval (corresponding to robust standard errors, clustered around hospital) around these estimates is shown. Sources: Healthcare Provider Cost Reporting Information System; Inpatient Prospective Payment System Payment Impact Files; NIH IMPAC II. 
Table B1: Effect of BBA and NIH Budget Doubling with Pre-1994 Hospital Grant Application Intensity

\begin{tabular}{|c|c|c|c|c|c|c|}
\hline & \multicolumn{2}{|c|}{ Grant Applications } & \multicolumn{2}{|c|}{ Grants Funded } & \multicolumn{2}{|c|}{ Publications } \\
\hline & (1) & (2) & (3) & (4) & (5) & (6) \\
\hline BBA Bite $\times$ Post BBA & $\begin{array}{c}19.109^{* * *} \\
(3.801)\end{array}$ & $\begin{array}{c}10.029^{* *} \\
(3.891)\end{array}$ & $\begin{array}{c}14.181^{* * *} \\
(3.048)\end{array}$ & $\begin{array}{l}6.059^{* *} \\
(2.933)\end{array}$ & $\begin{array}{l}8.783^{*} \\
(5.239)\end{array}$ & $\begin{array}{c}4.760 \\
(5.480)\end{array}$ \\
\hline RI $\times$ Post NIH Doubling & & $\begin{array}{c}4.739^{* * *} \\
(0.897)\end{array}$ & & $\begin{array}{c}4.239^{* * *} \\
(0.791)\end{array}$ & & $\begin{array}{l}2.100^{* *} \\
(0.678)\end{array}$ \\
\hline Elasticity (BBA Bite) & 0.061 & 0.032 & 0.048 & 0.021 & 0.028 & 0.015 \\
\hline Elasticity (RI) & & 0.017 & & 0.016 & & 0.007 \\
\hline Adjusted $R^{2}$ & 0.021 & 0.037 & 0.014 & 0.035 & 0.023 & 0.024 \\
\hline Meal & 0.757 & 0.7 & 0.44 & 0.445 & 2.012 & 2.012 \\
\hline $\mathrm{Nb}$. & 10,920 & 10,920 & 10,920 & 10,920 & 10,920 & 10,920 \\
\hline Nb. Hospitals & 780 & 780 & 780 & 780 & 780 & 780 \\
\hline
\end{tabular}

Notes: This table reports difference-in-differences estimates of the effect of the BBA and NIH budget on the number of grant applications, grants funded, and publications, in the teaching hospital sample over the period 1994-2007. Observations are at the hospital-year level. Outcomes are transformed with the inverse hyperbolic sine function. BBA Bite is a hospital's BBA Bite and RI a hospital's average number of annual publications between 1990-1991 divided by 1000. Estimates are from OLS regressions, and each coefficient is from a separate regression. Robust standard errors (clustered at the hospital level) are in parentheses. Sources: Healthcare Provider Cost Reporting Information System; Inpatient Prospective Payment System Payment Impact Files; NIH IMPAC II; PubMed, Web of Science.

$p<0.10,{ }^{* *} p<0.05,{ }^{* * *} p<0.001$. 
Table B2: Comparing Characteristics of Low and High Research Intensity Hospitals, 1992-1997

\begin{tabular}{|c|c|c|c|c|c|c|c|}
\hline & \multicolumn{3}{|c|}{$\begin{array}{c}\text { Low Research Intensity } \\
\text { Hospital Sample }\end{array}$} & \multicolumn{3}{|c|}{$\begin{array}{c}\text { High Research Intensity } \\
\text { Hospital Sample }\end{array}$} & \multirow{2}{*}{$\begin{array}{c}\begin{array}{c}\text { P-value from } \\
\text { T-test }\end{array} \\
\text { Diff. of Meant }\end{array}$} \\
\hline & Count & Mean & $\mathrm{SD}$ & Count & Mean & $\mathrm{SD}$ & \\
\hline \multicolumn{8}{|l|}{ Panel A. Hospital Characteristics } \\
\hline Discharges $(1,000 \mathrm{~s})$ & 440 & 12.02 & 6.39 & 340 & 20.61 & 10.44 & $0.000^{* * *}$ \\
\hline Inpatient Days (1,000s) & 440 & 65.96 & 36.91 & 340 & 121.61 & 66.85 & $0.000^{* * *}$ \\
\hline Medicare Teaching Payment $(\$ 1,000,000 \mathrm{~s})$ & 440 & 1.62 & 2.26 & 340 & 9.06 & 8.56 & $0.000^{* * *}$ \\
\hline Medicare Disproportionate Share Payment $(\$ 1,000,000 \mathrm{~s})$ & 440 & 1.63 & 2.09 & 340 & 4.32 & 4.33 & $0.000^{* * *}$ \\
\hline Total Revenue $(\$ 1,000,000 \mathrm{~s})$ & 428 & 180.5 & 105.93 & 337 & 378.41 & 235.16 & $0.000^{* * *}$ \\
\hline Beds & 440 & 301.18 & 139.24 & 340 & 483.21 & 228.11 & $0.000^{* * *}$ \\
\hline Residents and Interns & 418 & 31.87 & 35.78 & 339 & 176.99 & 152.04 & $0.000^{* * *}$ \\
\hline Pre-1992 Research (Number of Publications) & 440 & 0.71 & 0.74 & 340 & 68.69 & 132.18 & $0.000^{* * *}$ \\
\hline BBA Bite $(x 100)$ & 440 & 0.29 & 0.26 & 340 & 0.64 & 0.35 & $0.000^{* * *}$ \\
\hline Medicare Share of Discharges in 1995 (\$1,000s) & 440 & 0.36 & 0.13 & 340 & 0.3 & 0.12 & $0.000 * * *$ \\
\hline Medicare Share of Inpatient Days in 1995 & 440 & 0.47 & 0.15 & 340 & 0.38 & 0.14 & $0.000^{* * *}$ \\
\hline Medicare Price Per Discharge in $1995(\$ 1,000$ s) & 440 & 7.06 & 1.74 & 340 & 9.9 & 3.19 & $0.000^{* * *}$ \\
\hline \multicolumn{8}{|l|}{ Panel B. Grants } \\
\hline \multicolumn{8}{|l|}{ Number of Grant Applications } \\
\hline Total & 440 & 0.13 & 0.86 & 340 & 13.74 & 31.35 & $0.000 * * *$ \\
\hline New & 440 & 0.11 & 0.63 & 340 & 10.4 & 23.38 & $0.000^{* * *}$ \\
\hline Competitive Renewal & 440 & 0.02 & 0.25 & 340 & 3.34 & 8.09 & $0.000^{* * *}$ \\
\hline Novice Investigator & 440 & 0.03 & 0.45 & 340 & 5.85 & 13.97 & $0.000 * * *$ \\
\hline Seasoned Investigator & 440 & 0.07 & 0.43 & 340 & 7.01 & 15.62 & $0.000 * * *$ \\
\hline MD Principal Investigator & 440 & 0.05 & 0.33 & 340 & 5.16 & 12.31 & $0.000^{* * *}$ \\
\hline PhD Principal Investigator & 440 & 0.05 & 0.38 & 340 & 6.52 & 15.46 & $0.000^{* * *}$ \\
\hline MD-PhD Principal Investigator & 440 & 0.02 & 0.26 & 340 & 1.81 & 4.63 & $0.000^{* * *}$ \\
\hline \multicolumn{8}{|l|}{ Number of Grants Funded } \\
\hline Total & 440 & 0.03 & 0.24 & 340 & 4.15 & 10.47 & $0.000^{* * *}$ \\
\hline New & 440 & 0.02 & 0.14 & 340 & 2.73 & 6.86 & $0.000 * * *$ \\
\hline Competitive Renewal & 440 & 0.01 & 0.11 & 340 & 1.42 & 3.68 & $0.000^{* * *}$ \\
\hline Novice Investigator & 440 & 0.01 & 0.14 & 340 & 1.94 & 5.06 & $0.000^{* * *}$ \\
\hline Seasoned Investigator & 440 & 0.02 & 0.1 & 340 & 2.05 & 5.23 & $0.000 * * *$ \\
\hline MD Principal Investigator & 440 & 0.01 & 0.08 & 340 & 1.65 & 4.36 & $0.000^{* * *}$ \\
\hline PhD Principal Investigator & 440 & 0.01 & 0.11 & 340 & 1.9 & 4.9 & $0.000^{* * *}$ \\
\hline MD-PhD Principal Investigator & 440 & 0.00 & 0.07 & 340 & 0.56 & 1.7 & $0.000^{* * *}$ \\
\hline \multicolumn{8}{|l|}{ Panel C. Publications } \\
\hline Total & 440 & 0.93 & 1.27 & 340 & 78.27 & 155.31 & $0.000 * * *$ \\
\hline Citation Percentile: $\leq 25$ & 440 & 0.39 & 0.55 & 340 & 19.76 & 32.74 & $0.000^{* * *}$ \\
\hline Citation Percentile: $\overline{26-50}$ & 440 & 0.21 & 0.37 & 340 & 18.18 & 33.92 & $0.000^{* * *}$ \\
\hline Citation Percentile: 51-75 & 440 & 0.18 & 0.32 & 340 & 18.75 & 38.41 & $0.000 * * *$ \\
\hline Citation Percentile: $76-95$ & 440 & 0.11 & 0.21 & 340 & 16.7 & 39.12 & $0.000^{* * *}$ \\
\hline Citation Percentile: 96-99 & 440 & 0.02 & 0.08 & 340 & 3.8 & 10.53 & $0.000 * * *$ \\
\hline Citation Percentile: $>99$ & 440 & 0.01 & 0.03 & 340 & 1.08 & 3.36 & $0.000 * * *$ \\
\hline Cited in a Patent & 440 & 0.13 & 0.27 & 340 & 21.64 & 51.89 & $0.000^{* * *}$ \\
\hline Not Cited in a Patent & 440 & 0.8 & 1.09 & 340 & 56.64 & 104.73 & $0.000^{* * *}$ \\
\hline Disruptive & 440 & 0.07 & 0.15 & 340 & 2.99 & 5.03 & $0.000 * * *$ \\
\hline Consolidating & 440 & 0.76 & 1.07 & 340 & 71.64 & 145.05 & $0.000^{* * *}$ \\
\hline Laboratory Research: Pub. with Bench MeSH & 440 & 0.05 & 0.19 & 340 & 25.51 & 60.42 & $0.000^{* * *}$ \\
\hline Translational Research: Pub. with Translational MeSH & 440 & 0.15 & 0.32 & 340 & 21.03 & 44.49 & $0.000 * * *$ \\
\hline Inspiring Translational & 440 & 0.12 & 0.22 & 340 & 5.56 & 9.57 & $0.000^{* * *}$ \\
\hline Builds on Translational & 440 & 0.07 & 0.18 & 340 & 10.72 & 24.19 & $0.000^{* * *}$ \\
\hline Clinical Research: Clinical Trial Pub. & 440 & 0.17 & 0.28 & 340 & 7.58 & 13.1 & $0.000 * * *$ \\
\hline Clinical Research: Pub. with Clinical MeSH & 440 & 0.47 & 0.67 & 340 & 18.91 & 33 & $0.000^{* * *}$ \\
\hline \multicolumn{8}{|c|}{ Panel D. Clinical Outcomes (Risk-Adjusted Survival Rates [30 Days]) } \\
\hline Heart Attack & 385 & 0.88 & 0.04 & 315 & 089 & 0.05 & 0.019 \\
\hline Heart Failure & 385 & 0.95 & 0.02 & 315 & 0.96 & 0.02 & $0.000^{* * *}$ \\
\hline Pneumonia & 385 & 0.98 & 0.02 & 315 & 0.98 & 0.02 & 0.157 \\
\hline Hip/knee & 385 & 0.94 & 0.03 & 315 & 0.94 & 0.03 & 0.154 \\
\hline \multicolumn{8}{|c|}{$\begin{array}{l}\text { Notes: This table compares Low Research and High Research intensity hospitals in the teaching hospital sample between 1992-1997. A hospital is } \\
\text { defined as a High Research hospital if its average annual number publications between 1990-1991 is above the median. The number of hospitals in } \\
\text { the Low Research and High Research sample vary because of the large number of hospitals with 0 publications between 1990-1991. All variables } \\
\text { are measured yearly. For example, "Discharges (1000s)" is the average number of patients a hospital receives in a year. See Section III for detailec } \\
\text { variable descriptions. Sources: Azoulay, Greenblatt, and Heggeness (2021); Chandra et al. (2016); Funk and Owen-Smith (2017); Healthcare } \\
\text { Provider Cost Reporting Information System; Inpatient Prospective Payment System Payment Impact Files; NIH IMPAC II; Marx and Fueg } \\
\text { (2020); PubMed; Web of Science. }\end{array}$} \\
\hline
\end{tabular}


Table B3: Effect of BBA and NIH Budget Doubling Among High and Low BBA Bite Hospitals

\begin{tabular}{|c|c|c|c|c|c|c|}
\hline & \multicolumn{2}{|c|}{ Grant Applications } & \multicolumn{2}{|c|}{ Grants Funded } & \multicolumn{2}{|c|}{ Publications } \\
\hline & (1) & (2) & (3) & (4) & (5) & (6) \\
\hline High BBA Bite $\times$ Post BBA & $\begin{array}{c}0.105^{* * *} \\
(0.025)\end{array}$ & $\begin{array}{l}0.059^{* *} \\
(0.021)\end{array}$ & $\begin{array}{c}0.076^{* * *} \\
(0.018)\end{array}$ & $\begin{array}{l}0.047^{* *} \\
(0.015)\end{array}$ & $\begin{array}{l}0.075^{*} \\
(0.039)\end{array}$ & $\begin{array}{l}0.100^{* *} \\
(0.043)\end{array}$ \\
\hline High RI $\times$ Post NIH Doubling & & $\begin{array}{c}0.108^{* * *} \\
(0.025)\end{array}$ & & $\begin{array}{c}0.070^{* * *} \\
(0.019)\end{array}$ & & $\begin{array}{l}-0.059 \\
(0.044)\end{array}$ \\
\hline Elasticity (High BBA Bite) & 0.110 & 0.061 & 0.079 & 0.048 & 0.078 & 0.104 \\
\hline Elasticity (High RI) & & 0.114 & & 0.072 & & -0.058 \\
\hline Adjusted $R^{2}$ & 0.019 & 0.024 & 0.013 & 0.016 & 0.030 & 0.030 \\
\hline Mean & 0.751 & 0.75 & 0.439 & 0.439 & 1.991 & 1.991 \\
\hline $\mathrm{Nb}$. & 12,480 & 12,480 & 12,480 & 12,480 & 12,480 & 12,480 \\
\hline Nb. Hospitals & 780 & 780 & 780 & 780 & 780 & 780 \\
\hline
\end{tabular}

Notes: $\overline{\text { This table reports difference-in-differences estimates of the effect of the BBA and NIH budget doubling }}$ on the number of grant applications, grants funded, and publications, in the teaching hospital sample. Observations are at the hospital-year level. Outcomes are transformed with the inverse hyperbolic sine function. High BBA Bite and High RI are indicators for whether a hospital's BBA Bite and RI, respectively, are above the median. Estimates are from OLS regressions, and each coefficient is from a separate regression. A hospital's RI is the average annual number of publications between 1990-1992. Robust standard errors (clustered at the hospital level) are in parentheses. Sources: Healthcare Provider Cost Reporting Information System; Inpatient Prospective Payment System Payment Impact Files; NIH IMPAC II; PubMed, Web of Science.

${ }^{*} p<0.10,{ }^{* *} p<0.05,{ }^{* * *} p<0.001$. 
Table B4: Effect on the Number of Grants Funded

\begin{tabular}{|c|c|c|c|c|c|c|c|c|}
\hline & \multirow[b]{2}{*}{$\begin{array}{l}\text { Total } \\
(1)\end{array}$} & \multicolumn{2}{|c|}{ Grant Cycle } & \multicolumn{2}{|c|}{$\begin{array}{c}\text { Principal Investigator } \\
\text { Experience }\end{array}$} & \multicolumn{3}{|c|}{$\begin{array}{c}\text { Principal Investigator } \\
\text { Degree }\end{array}$} \\
\hline & & $\begin{array}{l}\text { New } \\
(2)\end{array}$ & $\begin{array}{c}\text { Renewal } \\
(3)\end{array}$ & $\begin{array}{l}\text { Novice } \\
(4)\end{array}$ & $\begin{array}{c}\text { Incumbent } \\
(5)\end{array}$ & $\begin{array}{l}\mathrm{MD} \\
(6)\end{array}$ & $\begin{array}{c}\mathrm{PhD} \\
(7)\end{array}$ & $\begin{array}{c}\mathrm{MD}-\mathrm{PhD} \\
(8)\end{array}$ \\
\hline BBA Bite $\times$ Post BBA & $\begin{array}{l}7.170^{* *} \\
(3.430)\end{array}$ & $\begin{array}{l}10.29^{* *} \\
(3.407)\end{array}$ & $\begin{array}{c}2.533 \\
(2.495)\end{array}$ & $\begin{array}{c}18.36^{* * *} \\
(3.245)\end{array}$ & $\begin{array}{l}1.529 \\
(2.987)\end{array}$ & $\begin{array}{l}5.672^{*} \\
(3.155)\end{array}$ & $\begin{array}{l}8.533^{* *} \\
(3.099)\end{array}$ & $\begin{array}{c}10.96^{* * *} \\
(2.683)\end{array}$ \\
\hline Elasticity & 0.021 & 0.033 & 0.012 & 0.063 & 0.006 & 0.023 & 0.031 & 0.083 \\
\hline Adjusted $R^{2}$ & 0.038 & 0.060 & 0.015 & 0.119 & 0.017 & 0.034 & 0.059 & 0.086 \\
\hline Diff. Wald test p-value & & \multicolumn{2}{|c|}{0.010} & \multicolumn{2}{|c|}{0.000} & & 0.399 & 0.144 \\
\hline Mean of Outcome & 0.439 & 0.382 & 0.246 & 0.325 & 0.312 & 0.285 & 0.300 & 0.159 \\
\hline Nb. Observations & 12,480 & 12,480 & 12,480 & 12,480 & 12,480 & 12,480 & 12,480 & 12,480 \\
\hline Nb. Hospitals & 780 & 780 & 780 & 780 & 780 & 780 & 780 & 780 \\
\hline
\end{tabular}

Notes: This table reports difference-in-differences estimates of the effect of the BBA on the number of grants funded, in the teaching hospital sample. Observations are at the hospital-year level. Outcomes are transformed with the inverse hyperbolic sine function. Column 1 presents results from OLS regressions. Columns 2-8 present estimates from seemingly unrelated regressions. Each coefficient is from a separate regression. As shown in equation (3), regressions include hospital fixed effects, year fixed effects, and RI $\times$ Post NIH Doubling, where RI is the average number of publications between 1990-1991 divided by 1000 . The elasticity of 0.021 implies that a one percentage increase in BBA Bite is associated on average with a 0.021 percent yearly increase in grant applications following the BBA's enactment. The fifth show $p$-values from Wald tests that compare coefficients on BBA Bite $\times$ After across different columns (Column 2 vs. Column 3; Column 4 vs. Column 5; Column 6 vs. Column 7; Column 6 vs. Column 8). Standard errors are in parentheses, and are clustered at the hospital level. Sources: Healthcare Provider Cost Reporting Information System; Inpatient Prospective Payment System Payment Impact Files; NIH IMPAC II.

${ }^{*} p<0.10,{ }^{* *} p<0.05,{ }^{* * *} p<0.001$. 


\section{Appendix C: Mapping Outcomes to Hospitals}

Appendix Figure C1 provides an example of how NIH grant IDs and Medicare provider IDs are allocated to hospitals and medical centers. The University of California, San Francisco medical center includes the Parnassus Heights Campus, Mt. Zion Hospital and Medical Center, and San Francisco General Hospital. Each of these locations has a unique Medicare provider number and therefore receive an independent Medicare payment. However, the three campuses share a single, common NIH institutional code. Our strategy consists of looking at each of the PI addresses affiliated with the UCSF NIH institutional code and allocating each PI (and grant) to one of the three hospitals. 


\section{Figure C1: Mapping Research Activity to Hospitals}

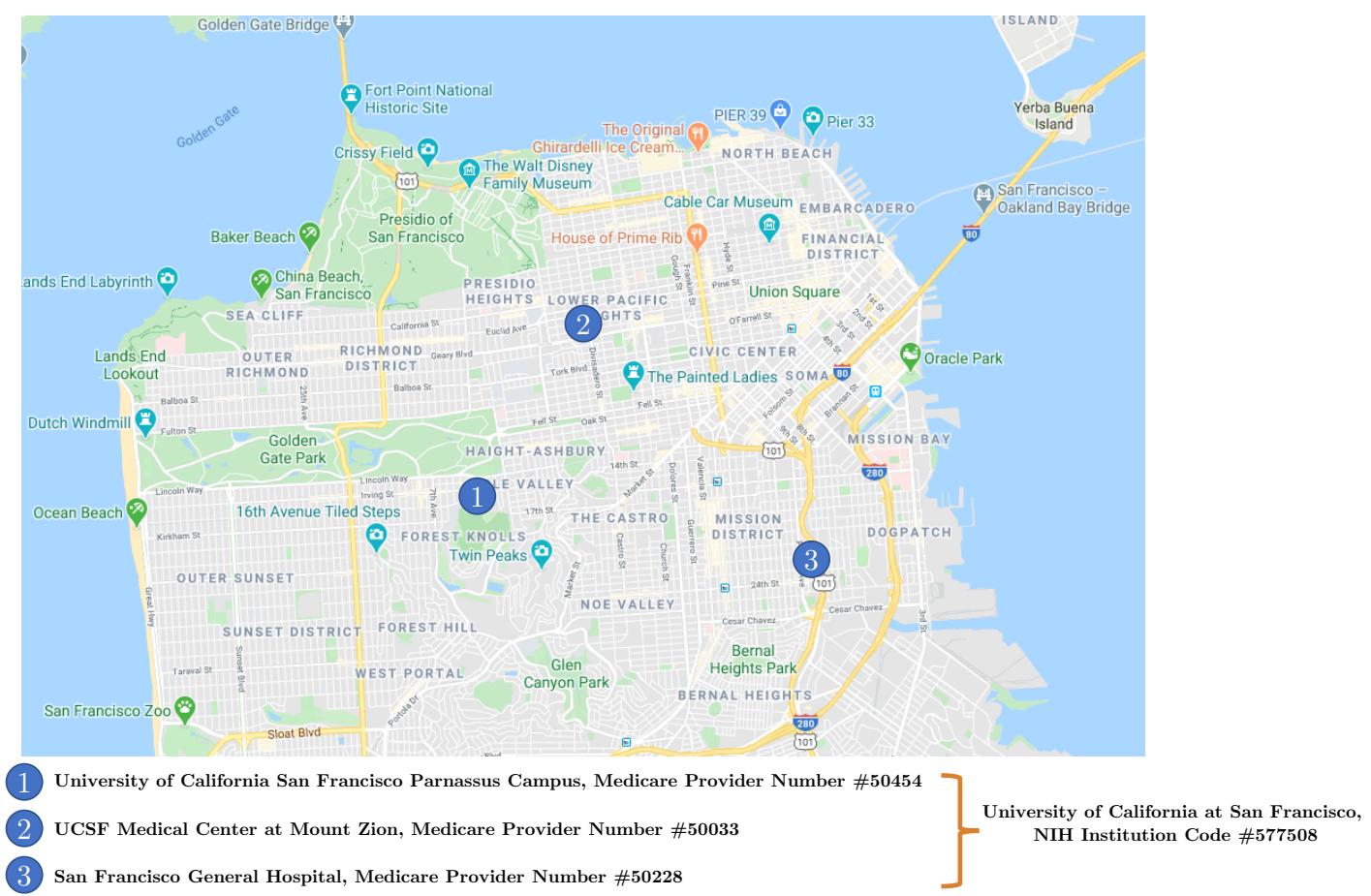

Notes: This figure shows how NIH grant IDs and Medicare provider IDs are allocated within University of California, San Francisco. Sources: Google Maps; Healthcare Provider Cost Reporting Information System; NIH IMPAC II. 


\section{Appendix D:}

\section{Academic Medical Center Sample Results}

This section provides summary statistics and regression results for the Academic Medical

Center sample of hospitals. See Section III for a description of how hospitals in this sample were identified. 
Figure D1: Distribution of BBA Bite

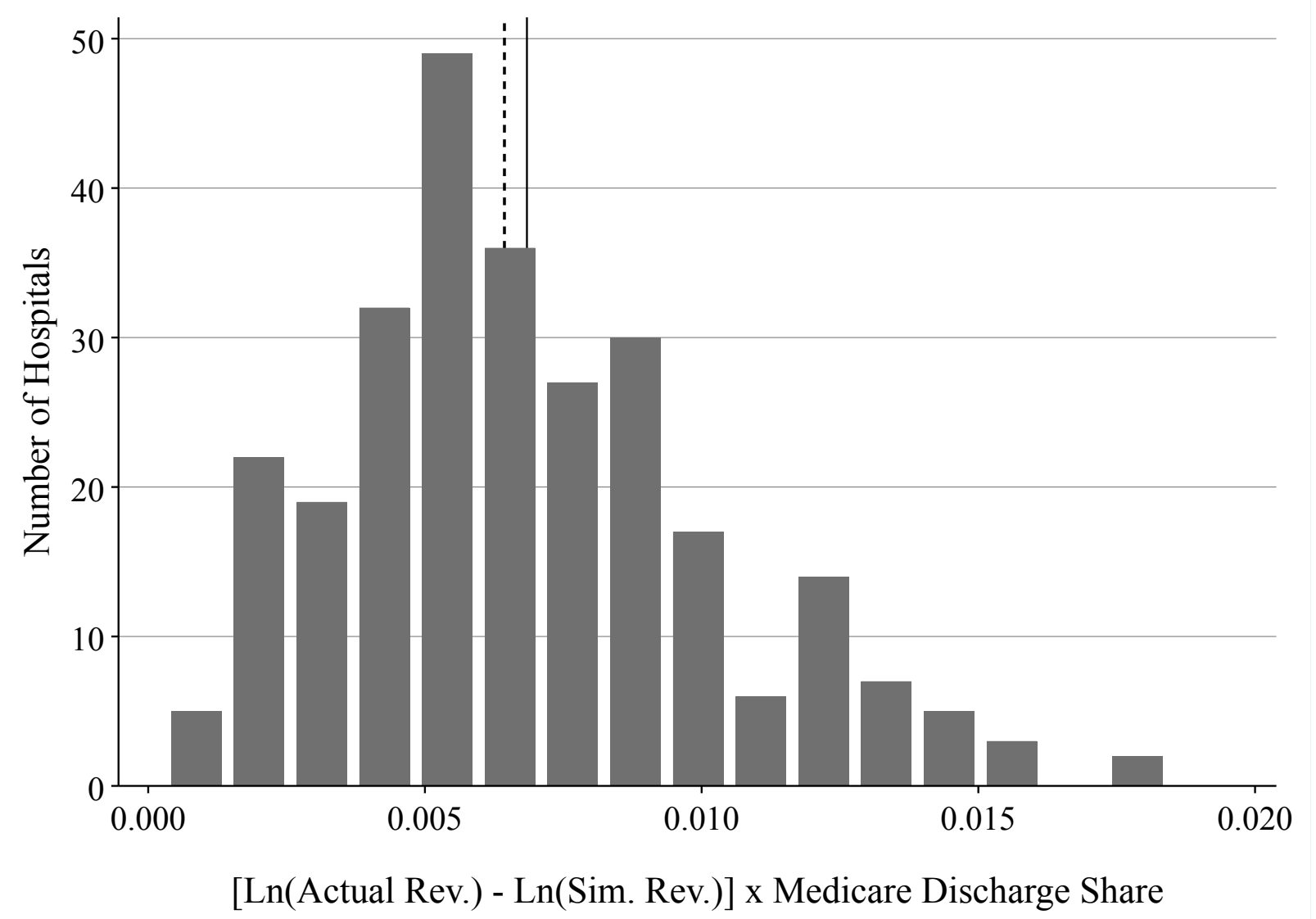

Notes: This figure shows a histogram of the BBA Bite, where BBA Bite is the difference between the simulated price per discharge and actual PPS price per discharge in 1995 in weighted by the Medicare share of discharges averaged over 1992-1995. The hospital sample used is the AMC sample. The solid line indicates the mean (0.0068) and the dotted lines indicates the median (0.0064). Sources: Healthcare Provider Cost Reporting Information System; Inpatient Prospective Payment System Payment Impact Files. 
Figure D2: Distribution of Research Intensity

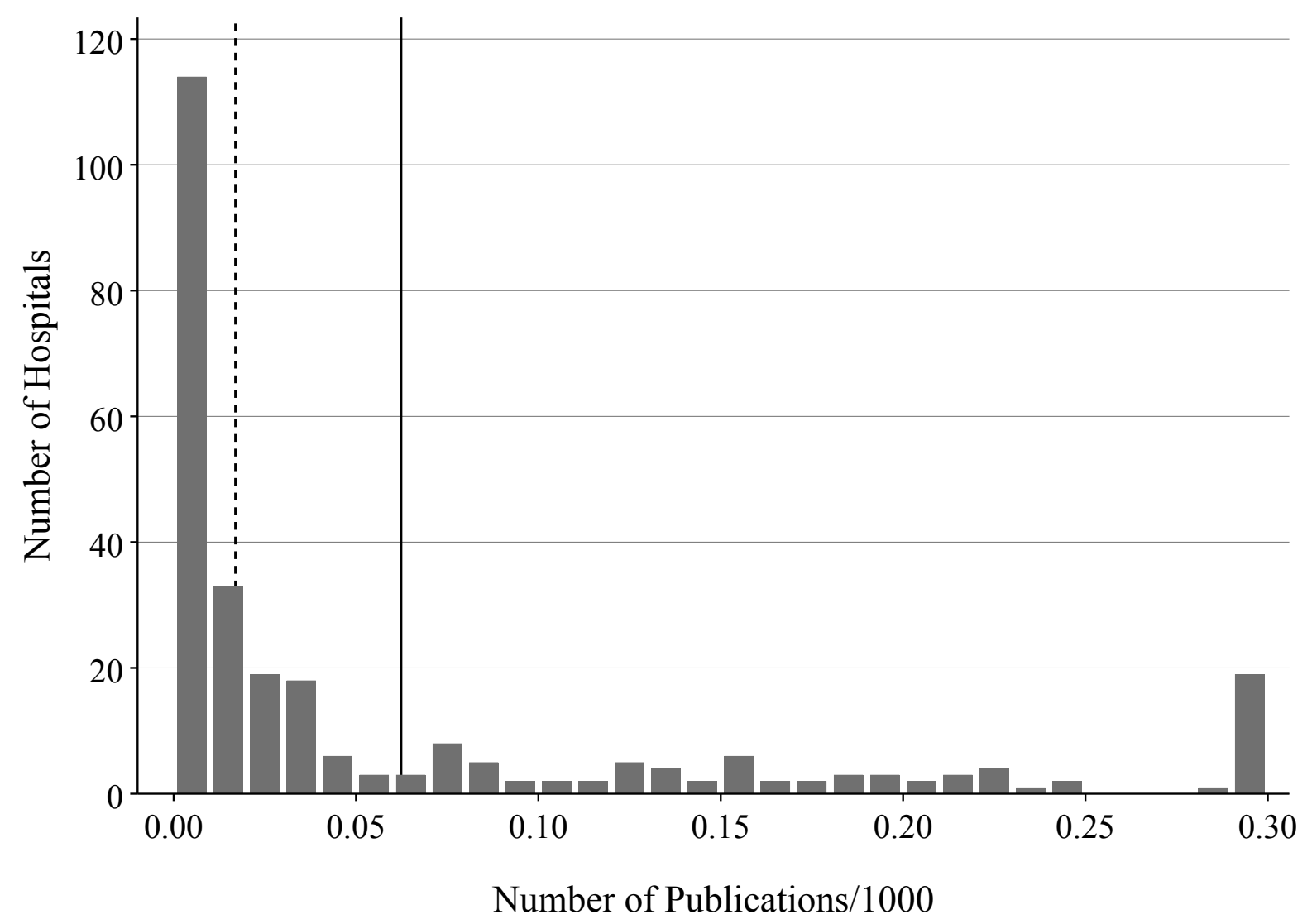

Notes: This figure shows a histogram of the Research Intensity, where Research Intensity is the annual number of publications averaged over 1990-1991 and divided by 1000. The hospital sample used is the AMC sample. The solid line indicates the mean $(0.062)$ of this variable and the dotted lines indicates the median (0.017). For ease of interpretation in this figure, hospitals with Research Intensity greater than 0.3 are recoded as 0.3. Sources: Healthcare Provider Cost Reporting Information System; Inpatient Prospective Payment System Payment Impact Files. 
Figure D3: Effect on the Number of Grant Applications

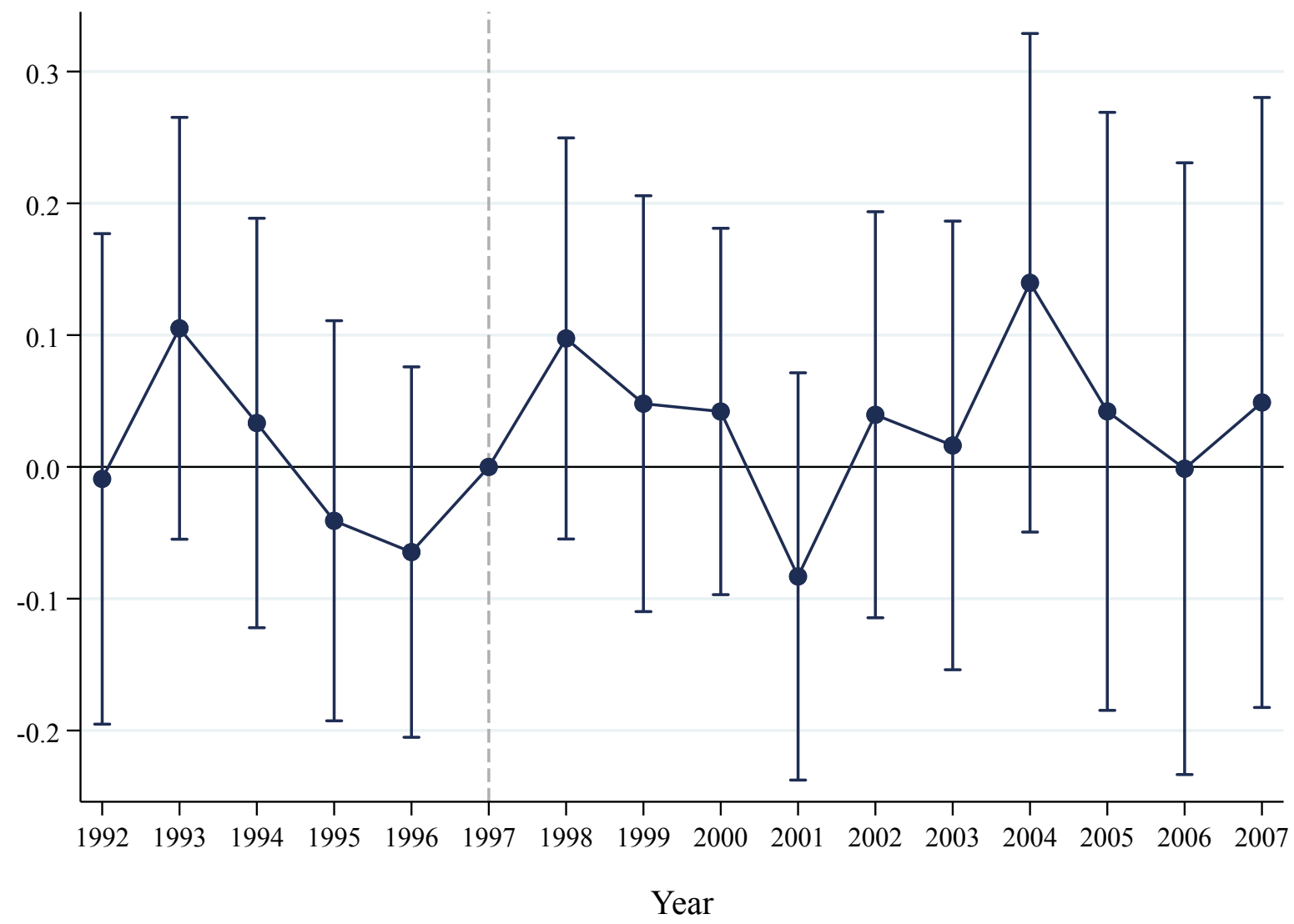

Notes: This figure plots the response in grant applications in the AMC sample. Each dot corresponds to OLS coefficient estimates stemming from the event study specification in which asinh(number of grant applications) is regressed onto year fixed effects, hospital fixed effects, interaction terms between an indicator for a hospital's High BBA Bite status interacted with the number of years before/after the BBA, as well as interaction terms between an indicator for a hospital's High RI status interacted with the number of years before/after the start of the NIH budget doubling. The 95\% confidence interval (corresponding to robust standard errors, clustered around hospital) around these estimates is shown. Sources: Healthcare Provider Cost Reporting Information System; Inpatient Prospective Payment System Payment Impact Files; NIH IMPAC II. 
Figure D4: Effect on the Number of Grants Funded

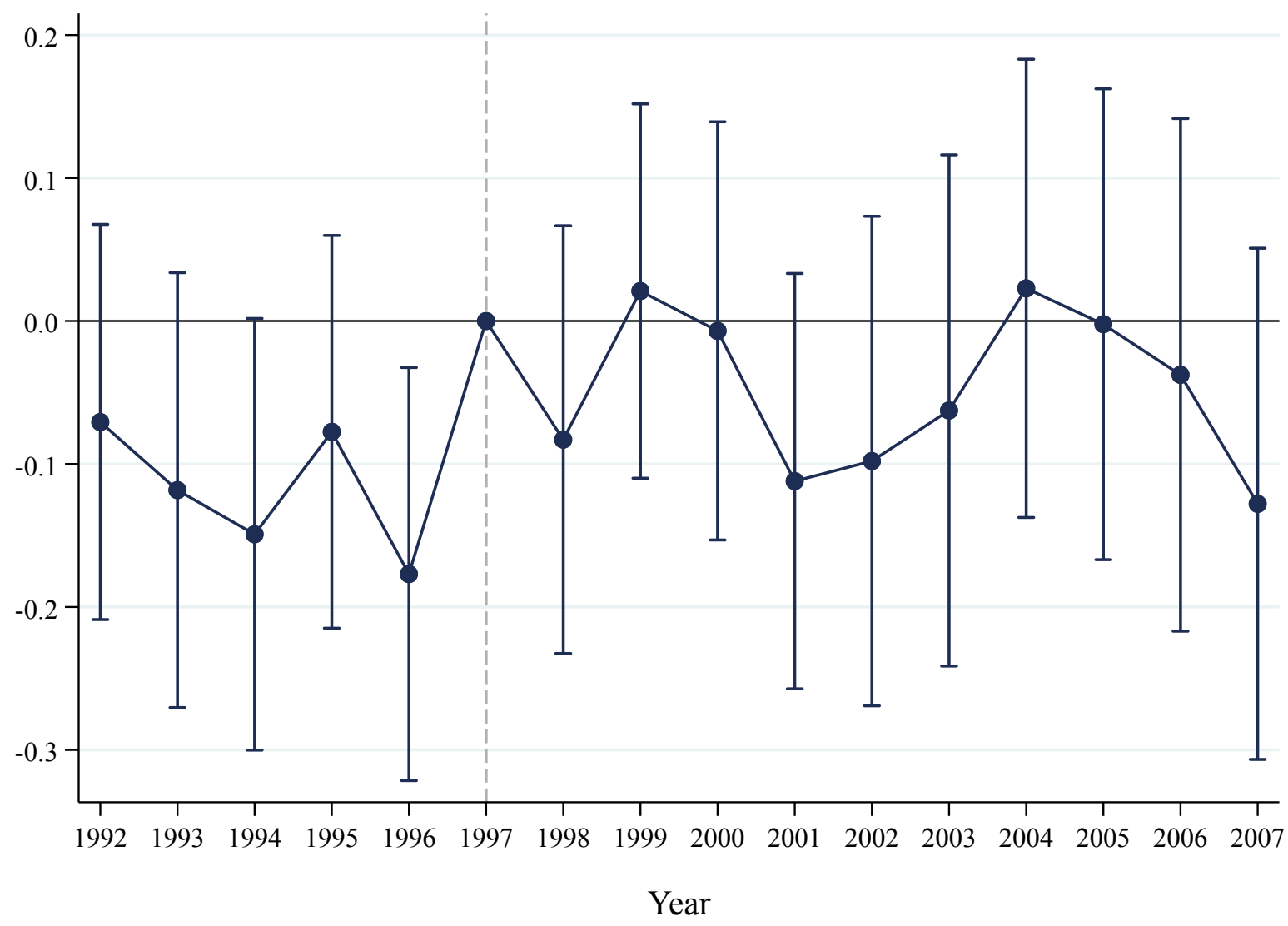

Notes: This figure plots the response in grants funded in the AMC sample. Each dot corresponds to OLS coefficient estimates stemming from the event study specification in which asinh(number of grants funded) is regressed onto year fixed effects, hospital fixed effects, interaction terms between an indicator for a hospital's High BBA Bite status interacted with the number of years before/after the BBA, as well as interaction terms between an indicator for a hospital's High RI status interacted with the number of years before/after the start of the NIH budget doubling. The 95\% confidence interval (corresponding to robust standard errors, clustered around hospital) around these estimates is shown. Sources: Healthcare Provider Cost Reporting Information System; Inpatient Prospective Payment System Payment Impact Files; NIH IMPAC II. 
Figure D5: Effect on the Number of Publications

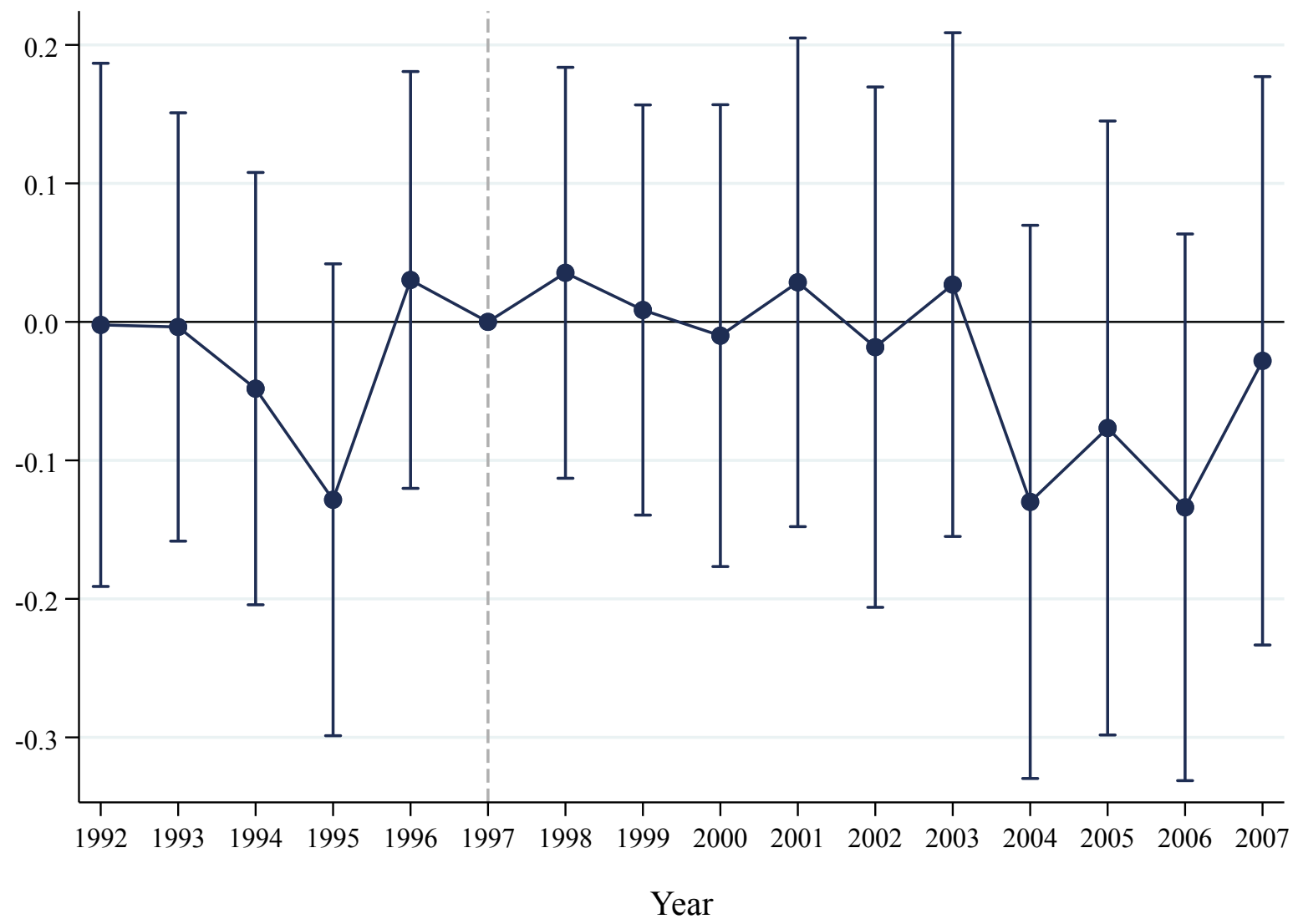

Notes: This figure plots the response in publications in the AMC sample. Each dot corresponds to OLS coefficient estimates stemming from the event study specification in which asinh(number of publications) is regressed onto year fixed effects, hospital fixed effects, interaction terms between an indicator for a hospital's High BBA Bite status interacted with the number of years before/after the BBA, as well as interaction terms between an indicator for a hospital's High RI status interacted with the number of years before/after the start of the NIH budget doubling. The 95\% confidence interval (corresponding to robust standard errors, clustered around hospital) around these estimates is shown. Sources: Healthcare Provider Cost Reporting Information System; Inpatient Prospective Payment System Payment Impact Files; NIH IMPAC II. 
Table D1: Comparing the Teaching Hospital and AMC Sample

\begin{tabular}{|c|c|c|c|c|c|c|c|}
\hline & \multicolumn{3}{|c|}{ Teaching Hospital Sample } & \multicolumn{3}{|c|}{ AMC Sample } & \multirow{2}{*}{$\begin{array}{c}\begin{array}{c}\text { P-value from } \\
\text { T-test }\end{array} \\
\text { Diff. of Meant }\end{array}$} \\
\hline & Count & Mean & $\mathrm{SD}$ & Count & Mean & $\mathrm{SD}$ & \\
\hline \multicolumn{8}{|l|}{ Panel A. Hospital Characteristics } \\
\hline Discharges $(1,000 \mathrm{~s})$ & 780 & 16.82 & 10.39 & 274 & 24.08 & 10.94 & $0.000^{* * *}$ \\
\hline Inpatient Days $(1,000 \mathrm{~s})$ & 780 & 89.84 & 60.25 & 274 & 134.1 & 64.64 & $0.000^{* * *}$ \\
\hline Medicare Teaching Payment $(\$ 1,000,000 \mathrm{~s})$ & 780 & 5.45 & 7.91 & 274 & 11.71 & 10.14 & $0.000^{* * *}$ \\
\hline Medicare Disproportionate Share Payment $(\$ 1,000,000 \mathrm{~s})$ & 780 & 3.97 & 4.54 & 274 & 6.62 & 5.49 & $0.000^{* * *}$ \\
\hline Total Revenue $(\$ 1,000,000 \mathrm{~s})$ & 765 & 486.94 & 410.95 & 274 & 759.2 & 498.32 & $0.000^{* * *}$ \\
\hline Beds & 780 & 360.54 & 202.23 & 274 & 497.32 & 216.19 & $0.000^{* * *}$ \\
\hline Residents and Interns & 767 & 101.92 & 139.35 & 274 & 213.45 & 168.61 & $0.000^{* * *}$ \\
\hline Pre-1992 Research (Number of Publications) & 780 & 30.34 & 93.5 & 274 & 76.57 & 139.21 & $0.000^{* * *}$ \\
\hline BBA Bite (x100) & 780 & 0.45 & 0.35 & 274 & 0.68 & 0.34 & $0.000^{* * *}$ \\
\hline Medicare Share of Discharges in $1995(\$ 1,000 \mathrm{~s})$ & 780 & 0.34 & 0.13 & 274 & 0.30 & 0.11 & $0.000^{* * *}$ \\
\hline Medicare Share of Inpatient Days in 1995 & 780 & 0.43 & 0.15 & 274 & 0.39 & 0.14 & $0.000^{* * *}$ \\
\hline Medicare Revenue Per Discharge in $1995(\$ 1,000$ s) & 780 & 8.3 & 2.85 & 274 & 10.09 & 3.11 & $0.000^{* * *}$ \\
\hline \multicolumn{8}{|l|}{ Panel B. Grants } \\
\hline \multicolumn{8}{|l|}{ Number of Grant Applications } \\
\hline Total & 780 & 8.82 & 32.97 & 274 & 23 & 50.83 & $0.000^{* * *}$ \\
\hline New & 780 & 7.15 & 26.43 & 274 & 18.63 & 40.72 & $0.000^{* * *}$ \\
\hline Competitive Renewal & 780 & 1.67 & 6.60 & 274 & 4.37 & 10.23 & $0.000^{* * *}$ \\
\hline Novice Investigators & 780 & 4.55 & 17.07 & 274 & 11.86 & 26.24 & $0.000^{* * *}$ \\
\hline Incumbent Investigators & 780 & 4.10 & 15.44 & 274 & 10.66 & 23.85 & $0.000^{* * *}$ \\
\hline MD Principal Investigators & 780 & 3.08 & 11.87 & 274 & 7.91 & 18.21 & $0.000^{* * *}$ \\
\hline PhD Principal Investigators & 780 & 4.26 & 16.22 & 274 & 11.25 & 25.3 & $0.000^{* * *}$ \\
\hline MD-PhD Principal Investigators & 780 & 1.36 & 5.81 & 274 & 3.55 & 9.07 & $0.000^{* * *}$ \\
\hline \multicolumn{8}{|l|}{ Number of Grants Funded } \\
\hline Total & 780 & 2.55 & 10.42 & 274 & 6.65 & 16.26 & $0.000^{* * *}$ \\
\hline New & 780 & 1.82 & 7.40 & 274 & 4.74 & 11.55 & $0.000^{* * *}$ \\
\hline Competitive Renewal & 780 & 0.73 & 3.05 & 274 & 1.90 & 4.75 & $0.000^{* * *}$ \\
\hline Novice Investigators & 780 & 1.40 & 5.74 & 274 & 3.66 & 8.93 & $0.000^{* * *}$ \\
\hline Incumbent Investigators & 780 & 1.11 & 4.62 & 274 & 2.90 & 7.24 & $0.000^{* * *}$ \\
\hline MD Principal Investigators & 780 & 0.96 & 4.05 & 274 & 2.49 & 6.30 & $0.000^{* * *}$ \\
\hline PhD Principal Investigators & 780 & 1.16 & 4.77 & 274 & 3.06 & 7.50 & $0.000^{* * *}$ \\
\hline MD-PhD Principal Investigators & 780 & 0.40 & 1.92 & 274 & 1.03 & 3.05 & $0.000^{* * *}$ \\
\hline \multicolumn{8}{|l|}{ Panel C. Publications } \\
\hline Total & 780 & 45.4 & 148.55 & 274 & 116.32 & 227.74 & $0.000^{* * *}$ \\
\hline Citation Percentile: $\leq 25$ & 780 & 11.19 & 31.02 & 274 & 28.29 & 46.17 & $0.000^{* * *}$ \\
\hline Citation Percentile: $\overline{26}-50$ & 780 & 10.41 & 32 & 274 & 26.65 & 48.56 & $0.000^{* * *}$ \\
\hline Citation Percentile: $51-75$ & 780 & 11.03 & 37.19 & 274 & 28.39 & 57.28 & $0.000^{* * *}$ \\
\hline Citation Percentile: $76-95$ & 780 & 9.84 & 37.25 & 274 & 25.39 & 58.2 & $0.000^{* * *}$ \\
\hline Citation Percentile: 96-99 & 780 & 2.27 & 9.98 & 274 & 5.88 & 15.84 & $0.000^{* * *}$ \\
\hline Citation Percentile: >99 & 780 & 0.67 & 3.18 & 274 & 1.74 & 5.11 & $0.000^{* * *}$ \\
\hline Cited in a Patent & 780 & 11.34 & 43.4 & 274 & 29.46 & 68.03 & $0.000^{* * *}$ \\
\hline Not Cited in a Patent & 780 & 34.07 & 106.05 & 274 & 86.87 & 161.22 & $0.000^{* * *}$ \\
\hline Consolidating: Disruption Index Percentile ; 95 & 780 & 1.69 & 4.86 & 274 & 4.26 & 7.34 & $0.000^{* * *}$ \\
\hline Disruptive: Disruption Index Percentile ¿ 95 & 780 & 40.72 & 135.6 & 274 & 104.36 & 208.26 & $0.000^{* * *}$ \\
\hline Laboratory Research: Pub. with Bench MeSH & 780 & 12.41 & 47.18 & 274 & 32.43 & 73.46 & $0.000^{* * *}$ \\
\hline Translational & 780 & 12.34 & 42.86 & 274 & 32.08 & 66.29 & $0.000^{* * *}$ \\
\hline Inspiring Translational & 780 & 3.83 & 11.94 & 274 & 9.67 & 18.28 & $0.000^{* * *}$ \\
\hline Builds on Translational & 780 & 6.40 & 23.48 & 274 & 16.64 & 36.68 & $0.000^{* * *}$ \\
\hline Clinical Research: Clinical Trial Pub & 780 & 5.43 & 16.94 & 274 & 13.66 & 25.93 & $0.000^{* * *}$ \\
\hline Clinical Research: Pub. with Other Clinical MeSH & 780 & 11.54 & 34.8 & 274 & 28.89 & 52.96 & $0.000^{* * *}$ \\
\hline \multicolumn{8}{|c|}{ Panel D. Clinical Outcomes (Risk-Adjusted Survival Rates [30 Days]) } \\
\hline Heart Attack & 700 & 0.90 & 0.03 & 264 & 0.91 & 0.04 & 0.147 \\
\hline Heart Failure & 700 & 0.96 & 0.02 & 264 & 0.97 & 0.02 & $0.001^{* *}$ \\
\hline Pneumonia & 700 & 0.98 & 0.02 & 264 & 0.98 & 0.02 & 0.106 \\
\hline Hip/knee & 700 & 0.95 & 0.02 & 264 & 0.95 & 0.02 & 0.428 \\
\hline
\end{tabular}


Table D2: Effect on the Number of Grant Applications

\begin{tabular}{|c|c|c|c|c|c|c|c|c|}
\hline & \multirow[b]{2}{*}{$\begin{array}{c}\text { Total } \\
(1)\end{array}$} & \multicolumn{2}{|c|}{ Grant Cycle } & \multicolumn{2}{|c|}{$\begin{array}{c}\text { Principal Investigator } \\
\text { Experience } \\
\end{array}$} & \multicolumn{3}{|c|}{$\begin{array}{c}\text { Principal Investigator } \\
\text { Degree }\end{array}$} \\
\hline & & $\begin{array}{l}\text { New } \\
(2)\end{array}$ & $\begin{array}{c}\text { Renewal } \\
(3)\end{array}$ & $\begin{array}{l}\text { Novice } \\
(4)\end{array}$ & $\begin{array}{l}\text { Incumbent } \\
(5)\end{array}$ & $\begin{array}{c}\mathrm{MD} \\
(6)\end{array}$ & $\begin{array}{c}\mathrm{PhD} \\
(7)\end{array}$ & $\begin{array}{l}\text { MD-PhD } \\
\quad(8)\end{array}$ \\
\hline BBA Bite $\times$ Post BBA & $\begin{array}{c}7.218 \\
(9.369)\end{array}$ & $\begin{array}{l}10.02 \\
(9.496)\end{array}$ & $\begin{array}{l}5.249 \\
(6.728)\end{array}$ & $\begin{array}{c}34.67^{* * *} \\
(8.885)\end{array}$ & $\begin{array}{l}-7.449 \\
(8.994)\end{array}$ & $\begin{array}{l}11.94 \\
(9.914)\end{array}$ & $\begin{array}{c}9.632 \\
(8.764)\end{array}$ & $\begin{array}{l}19.53^{* *} \\
(7.801)\end{array}$ \\
\hline Elasticity & 0.031 & 0.043 & 0.023 & 0.149 & -0.032 & 0.051 & 0.041 & 0.087 \\
\hline Adjusted $R^{2}$ & 0.058 & 0.084 & 0.017 & 0.202 & 0.037 & 0.047 & 0.095 & 0.132 \\
\hline Diff. Wald test p-value & & \multicolumn{2}{|c|}{0.584} & \multicolumn{2}{|r|}{0.000} & & 0.820 & 0.492 \\
\hline Mean of Outcome & 1.831 & 1.725 & 0.957 & 1.369 & 1.453 & 1.290 & 1.348 & 0.842 \\
\hline Nb. Observations & 4,384 & 4,384 & 4,384 & 4,384 & 4,384 & 4,384 & 4,384 & 4,384 \\
\hline Nb. Hospitals & 274 & 274 & 274 & 274 & 274 & 274 & 274 & 274 \\
\hline
\end{tabular}

Notes: This table reports difference-in-differences estimates of the effect of the BBA on the number of grant applications, in the AMC sample. Observations are at the hospital-year level. Outcomes are transformed with the inverse hyperbolic sine function. Column 1 presents results from OLS regressions. As shown in equation (3), regressions include hospital fixed effects, year fixed effects, and RI $\times$ Post NIH Doubling, where $\mathrm{RI}$ is the average number of publications between 1990-1991 divided by 1000. Columns 2-8 present estimates from seemingly unrelated regressions. Each coefficient is from a separate regression. The elasticity of 0.031 implies that a one percentage increase in BBA Bite is associated on average with a 0.031 percent yearly increase in grant applications following the BBA's enactment. The fourth row and eighth row show $p$-values from Wald tests that compare coefficients on BBA Bite $\times$ Post BBA across different columns (Column 2 vs. Column 3; Column 4 vs. Column 5; Column 6 vs. Column 7; Column 6 vs. Column 8). Robust standard errors (clustered at the hospital level) are in parentheses. Sources: Healthcare Provider Cost Reporting Information System; Inpatient Prospective Payment System Payment Impact Files; NIH IMPAC II.

${ }^{*} p<0.10,{ }^{* *} p<0.05,{ }^{* * *} p<0.001$. 
Table D3: Effect on the Number of Grants Funded

\begin{tabular}{|c|c|c|c|c|c|c|c|c|}
\hline & \multirow[b]{2}{*}{$\begin{array}{l}\text { Total } \\
(1)\end{array}$} & \multicolumn{2}{|c|}{ Grant Cycle } & \multicolumn{2}{|c|}{$\begin{array}{c}\text { Principal Investigator } \\
\text { Experience }\end{array}$} & \multicolumn{3}{|c|}{$\begin{array}{c}\text { Principal Investigator } \\
\text { Degree }\end{array}$} \\
\hline & & $\begin{array}{l}\text { New } \\
(2)\end{array}$ & $\begin{array}{c}\text { Renewal } \\
(3)\end{array}$ & $\begin{array}{l}\text { Novice } \\
(4)\end{array}$ & $\begin{array}{c}\text { Incumbent } \\
(5)\end{array}$ & $\begin{array}{l}\mathrm{MD} \\
(6)\end{array}$ & $\begin{array}{c}\mathrm{PhD} \\
(7)\end{array}$ & $\begin{array}{c}\mathrm{MD}-\mathrm{PhD} \\
(8)\end{array}$ \\
\hline BBA Bite $\times$ Post BBA & $\begin{array}{c}8.594 \\
(7.795)\end{array}$ & $\begin{array}{c}12.13 \\
(7.498)\end{array}$ & $\begin{array}{l}7.416 \\
(5.840)\end{array}$ & $\begin{array}{c}27.28^{* * *} \\
(6.816)\end{array}$ & $\begin{array}{l}-0.858 \\
(6.774)\end{array}$ & $\begin{array}{c}8.755 \\
(7.286)\end{array}$ & $\begin{array}{l}11.51^{*} \\
(6.817)\end{array}$ & $\begin{array}{c}19.60^{* * *} \\
(5.611)\end{array}$ \\
\hline Elasticity & 0.037 & 0.053 & 0.036 & 0.121 & -0.004 & 0.040 & 0.052 & 0.117 \\
\hline Adjusted $R^{2}$ & 0.046 & 0.070 & 0.016 & 0.143 & 0.027 & 0.038 & 0.067 & 0.096 \\
\hline Diff. Wald test p-value & & \multicolumn{2}{|c|}{0.446} & \multicolumn{2}{|c|}{0.000} & & 0.696 & 0.192 \\
\hline Mean of Outcome & 1.117 & 0.973 & 0.637 & 0.838 & 0.799 & 0.731 & 0.770 & 0.411 \\
\hline Nb. Observations & 4,384 & 4,384 & 4,384 & 4,384 & 4,384 & 4,384 & 4,384 & 4,384 \\
\hline Nb. Hospitals & 274 & 274 & 274 & 274 & 274 & 274 & 274 & 274 \\
\hline
\end{tabular}

Notes: This table reports difference-in-differences estimates of the effect of the BBA on the number of grants funded, in the AMC sample. Observations are at the hospital-year level. Outcomes are transformed with the inverse hyperbolic sine function. Column 1 presents results from OLS regressions. As shown in equation (3), regressions include hospital fixed effects, year fixed effects, and RI $\times$ Post NIH Doubling, where RI is the average number of publications between 1990-1991 divided by 1000. Columns 2-8 present estimates from seemingly unrelated regressions. Each coefficient is from a separate regression. The elasticity of 0.037 implies that a one percentage increase in BBA Bite is associated on average with a 0.037 percent yearly increase in grants funded following the BBA's enactment. The fourth row and eighth row show $p$-values from Wald tests that compare coefficients on BBA Bite $\times$ Post BBA across different columns (Column 2 vs. Column 3; Column 4 vs. Column 5; Column 6 vs. Column 7; Column 6 vs. Column 8). Robust standard errors (clustered at the hospital level) are in parentheses. Sources: Healthcare Provider Cost Reporting Information System; Inpatient Prospective Payment System Payment Impact Files; NIH IMPAC II.

${ }^{*} p<0.10,{ }^{* *} p<0.05,{ }^{* * *} p<0.001$. 


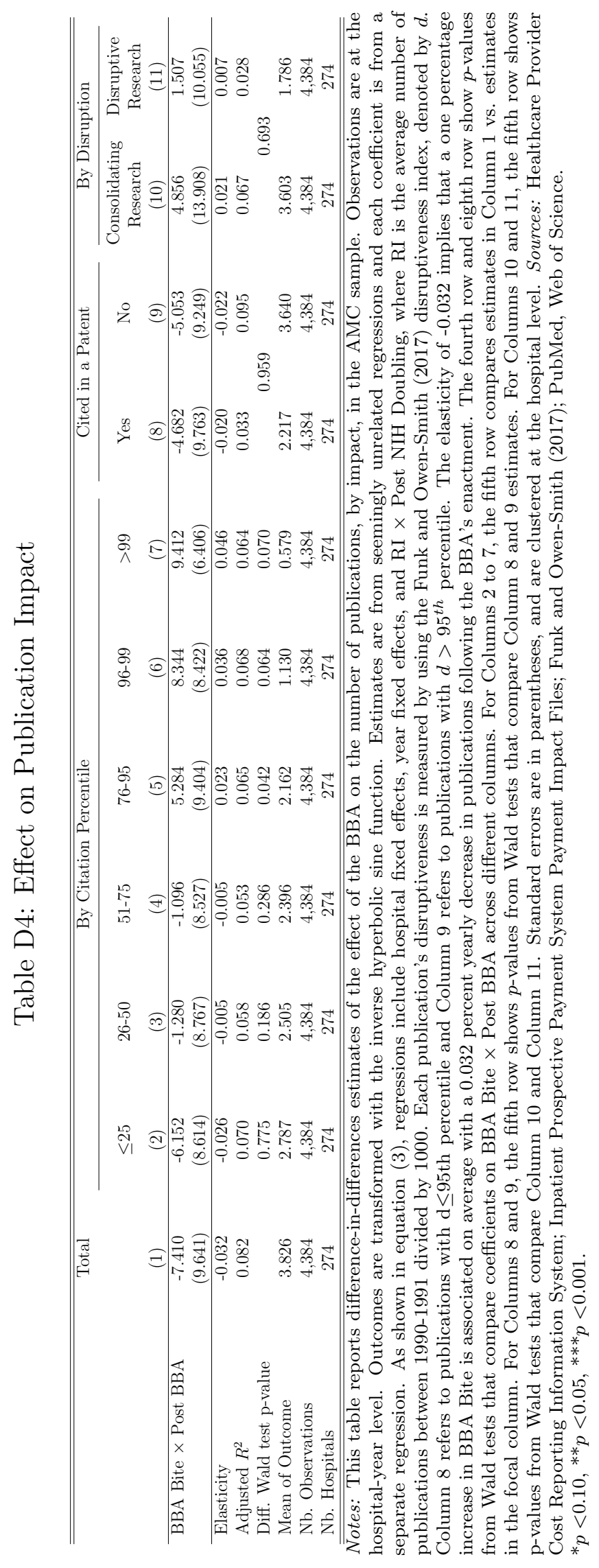

xxix 
Table D5: Effect on Publication Composition

\begin{tabular}{|c|c|c|c|c|c|c|}
\hline & \multirow{2}{*}{$\begin{array}{c}\begin{array}{c}\text { Laboratory } \\
\text { Research }\end{array} \\
\text { Bench } \\
\text { MeSH }\end{array}$} & \multicolumn{3}{|c|}{ Translational Research } & \multicolumn{2}{|c|}{ Clinical Research } \\
\hline & & $\begin{array}{c}\text { Translational } \\
\text { MeSH } \\
(2)\end{array}$ & $\begin{array}{c}\text { Builds on } \\
\text { Translational } \\
\text { MeSH } \\
(3)\end{array}$ & $\begin{array}{c}\text { Inspiring } \\
\text { Translational } \\
\text { MeSH } \\
(4)\end{array}$ & $\begin{array}{c}\text { Clinical Trials } \\
\text { (5) }\end{array}$ & $\begin{array}{c}\text { Other } \\
(6)\end{array}$ \\
\hline BBA Bite $\times$ Post BBA & $\begin{array}{l}-10.108 \\
(10.446)\end{array}$ & $\begin{array}{c}0.143 \\
(9.182) \\
\end{array}$ & $\begin{array}{c}19.614^{* *} \\
(8.404)\end{array}$ & $\begin{array}{l}10.504 \\
(8.491) \\
\end{array}$ & $\begin{array}{c}18.127^{* *} \\
(8.527)\end{array}$ & $\begin{array}{l}-1.798 \\
(9.274) \\
\end{array}$ \\
\hline Elasticity & -0.043 & 0.001 & 0.084 & 0.045 & 0.078 & -0.008 \\
\hline Adjusted $R^{2}$ & 0.001 & 0.045 & 0.157 & 0.061 & 0.199 & 0.073 \\
\hline Mean of Outcome & 2.005 & 2.400 & 1.781 & 1.874 & 2.061 & 2.829 \\
\hline Nb. Observations & 4,384 & 4,384 & 4,384 & 4,384 & 4,384 & 4,384 \\
\hline Nb. Hospitals & 274 & 274 & 274 & 274 & 274 & 274 \\
\hline
\end{tabular}

Notes: This table reports difference-in-differences estimates of the effect of the BBA on bench, translational, and clinical trial research in hospitals, in the AMC sample. Observations are at the hospital-year level. Outcomes are transformed with the inverse hyperbolic sine function. Estimates are from OLS regressions and each coefficient is from a separate regression. As shown in equation (3), regressions include hospital fixed effects, year fixed effects, and RI $\times$ Post NIH Doubling, where RI is the average number of publications between 1990-1991 divided by 1000. Column 1 refers to publications that are not disease-oriented, are not clinical trial publications, and rely on either a molecular biology technique, a model organism, cellular structures and macromolecules, or biochemical and cellular processes (based on MeSH terms). Column 2 refers to publications that are disease-oriented, are not clinical trial publications, and rely on either a molecular biology technique, a model organism, cellular structures and macromolecules, or biochemical and cellular processes (based on MeSH terms). Column 3 refers to publications that report the results of a clinical trials, or are tagged by a human MeSH term and also cite a translational publication. Column 4 refers to publications that are translational and is cited by a clinical trial publication (or one that contains a human MeSH term). Column 5 refers to publications that are indicated as clinical trials based on MeSH terms or the publication type field in PubMed. Finally, Column 6 refers to publications that are disease-oriented, not clinical trials, and not tagged by any bench MeSH keywords. The elasticity of -0.043 implies that a one percentage increase in BBA Bite is associated on average with a 0.043 percent yearly decrease in publications following the BBA's enactment. Standard errors are in parentheses, and are clustered at the hospital level. Sources: Healthcare Provider Cost Reporting Information System; Inpatient Prospective Payment System Payment Impact Files; Azoulay, Greenblatt, and Heggeness (2021); Marx and Fuegi (2020); PubMed; Web of Science.

${ }^{*} p<0.10,{ }^{* *} p<0.05,{ }^{* * *} p<0.001$. 
Table D6: Effect on Clinical Outcomes

(Changes in Risk-adjusted Survival Rates)

\begin{tabular}{lcccc}
\hline \hline & Heart Attack & Heart Failure & Hip/Knee & Pneumonia \\
& $(1)$ & $(2)$ & $(3)$ & $(4)$ \\
\hline BBA Bite & -0.0903 & -0.1854 & 0.3631 & -0.3402 \\
& $(0.8047)$ & $(0.4799)$ & $(0.4726)$ & $(0.5631)$ \\
Ln(Discharges in 1995) & 0.0064 & $0.0086^{* *}$ & 0.0016 & $0.0108^{* *}$ \\
& $(0.0053)$ & $(0.0033)$ & $(0.0036)$ & $(0.0038)$ \\
Adjusted $R^{2}$ & -0.0025 & 0.0214 & -0.0049 & 0.0232 \\
\hline Mean of Outcome & 0.0268 & 0.0103 & 0.0010 & 0.0175 \\
Nb. Observations & 264 & 264 & 264 & 264 \\
\hline
\end{tabular}

Notes: This table displays the effect on changes in risk-adjusted survival rates among hospitals in the AMC sample. The hospital sample used is the subset of the teaching hospital sample that is matched to the clinical outcomes dataset from Chandra et al. (2016). Observations are at the hospital-level. Outcomes are the difference in average survival rates between the post-BBA time period and the pre-BBA time period. Estimates are from OLS regressions and each coefficient is from a separate regression. Standard errors are in parentheses and robust. Sources: Healthcare Provider Cost Reporting Information System; Inpatient Prospective Payment System Payment Impact Files; Chandra et al. (2016).

${ }^{*} p<0.10,{ }^{* *} p<0.05,{ }^{* * *} p<0.001$. 


\section{Appendix E: Assessing the Doubling of the NIH Budget}

In this appendix, we document evidence that the our main results are primarily driven by the BBA and not the doubling of the NIH budget.

Documenting the Doubling of the NIH Budget. As described in Section IV, the doubling of the NIH budget between 1998 to 2003 presents a potential threat to identification. To begin, we use number of NIH grants as a proxy for NIH funding supply. Appendix Figure E1 shows trends in total NIH grants awarded to hospitals and non-hospitals by year for each year from 1980 to 2007. The figure illustrates that the total number of grants funded and the total amount of grant dollars awarded increased markedly from 1998 to 2003. Calculating the average amount of grant dollars awarded each year, we find that approximately $\$ 3.8$ billion is awarded each year before 1998 and $\$ 4.9$ billion is allocated each year on/after 1998 .

Assessing the Age-Bias of NIH Budget Doubling. In this section, we explore whether the doubling of the NIH budget is "age-biased." The key idea behind this test is that if the $\mathrm{NIH}$ budget increase was primarily directed towards research in conditions affecting elderly populations (e.g., Alzheimer's disease), hospitals that primarily conducted more research in elderly populations (e.g., high BBA Bite hospitals) may disproportionately benefit from the NIH budget doubling, biasing our resulting BBA estimates upwards.

First, we characterize NIH-related funding for research primarily conducted in conditions disproportionately affecting children (i.e., "Medicare-Averse" research), the elderly (i.e., "Medicare-Friendly" research), and individuals of all ages (i.e., "Medicare-Neutral" research). To categorize each NIH grant, we begin by identifying the set of Medicare-Averse, Medicare-Friendly, and Medicare-Neutral diseases as measured by ICD9 codes. For each ICD9 code, we calculate the share of individuals diagnosed with the focal condition and covered by Medicare using the 1996 and 1997 Medical Expenditure Panel Survey (MEPS), a nationally representative survey of the U.S. civilian non-institutionalized population.

Next, we assign each ICD9 code to one of four mutually exclusive "age-bias" bins based on their "ICD9 Medicare Share": ICD9 codes that fall in the bottom tenth percentile of the ICD9 Medicare Share distribution (i.e., ICD9 codes that are "Medicare-Averse"); ICD9 codes that fall between the fifty-fith and sixty-fifth percentile (i.e., ICD9 codes that are "Medicare-Neutral"); ICD9 codes that fall above the ninetieth percentile (i.e., ICD9 codes that are "Medicare-Friendly"); and ICD9 codes that fall in none of the previous bins. Focusing on the first three ICD9 code bins, we keep the top 15 ICD9 codes (based on the 
total number of individuals diagnosed with the focal ICD9 code in 1996 and 1997) in each ICD9 code bin. This results in a total of 45 unique ICD9 codes (15 in each ICD9 code bin).

We link MeSH terms to each ICD9 code and "age-bias" bin using the National Library of Medicine's Medical Text Indexer (MTI), a natural language processing tool which enables researchers to map text onto the MeSH controlled thesaurus. ${ }^{i}$ This results in 93 unique MeSH terms. As expected, MeSH terms in the Medicare-Averse category correspond to diseases that primarily afflict children, such as: Chickenpox and Measles. In contrast, MeSH terms in the Medicare-Friendly category include Glaucoma and Parkinson's Disease. MedicareNeutral MeSH terms include diseases that are less biased towards children or the elderly, such as Psoriasis and Cystitis.

Using the Medicare-Averse, Medicare-Neutral, and Medicare-Friendly MeSH categories, we categorize each grant. ${ }^{\text {ii }}$ In particular, we map words in the grant title and abstract to terms from the MeSH thesaurus by using the MTI. Next, we categorize a grant as being Medicare-Averse if it is tagged with at least one Medicare-Averse MeSH term. A grant is considered Medicare-Neutral if it is not Medicare-Averse and is tagged with at least one Medicare-Neutral MeSH term. Finally, we tag each grant that is not Medicare-Averse, not Medicare-Neutral, and contains at least one Medicare-Friendly MeSH term. Any duplicates (i.e., grants that are allocated to a MeSH category more than once) are dropped. This results in 2,954 distinct grants that are Medicare-Averse, 6,012 distinct grants that are MedicareNeutral, and 14,677 grants that are Medicare-Friendly.

Appendix Figure E2 documents the total amount of grant dollars awarded to hospitals in each year from 1992 to 2007 for the three MeSH categories. Looking to trends in the total number of grants funded (Panel A), we see clear evidence that the NIH awards a relatively large number of grants for Medicare-Friendly research as compared to Medicare-Averse or Medicare-Neutral research. The three groups follow each other quite closely in trends from 1992 to 1997. Between 1998 to 2004, the trends diverge with there being a disproportionate increase in Medicare-Friendly grants. Similar patterns occur among the total amount of grant dollars funds awarded (Panel B).

Notably, both graphs exhibit a significant decrease in 1996. This is likely related to the underlying data construction: grants in 1996 were linked to fewer MeSH terms on average (4.12 vs. 9.38 across the whole sample). As a result, grants in 1996 had a lower likelihood of being categorized as Medicare-Averse, Medicare-Neutral, or Medicare-Friendly. Despite this

\footnotetext{
${ }^{i}$ See: https://ii.nlm.nih.gov/MTI/.

${ }^{i i}$ Downloaded from the NIH Reporter web site at https://exporter.nih.gov/crisp_catalog.aspx.
} 
data limitation, the divergence in Medicare-Friendly grants-particularly in the total number of grants funded-is striking.

Finally, to empirically assess whether the trends across the three types of research are similar, we estimate:

$$
y_{t}=\beta 1998 \text { to } 2003_{t}+\varepsilon_{t}
$$

where $y_{t}$ is the total number of NIH grants funded or the total amount of grant dollars awarded in year $t$ and 1998to2003t is an indicator equal to 1 for years between 1998 and 2003, inclusive. Following our main analysis on the effect of the BBA on subsequent hospital research outcomes, we transform outcomes with the inverse hyperbolic sine function and perform our estimation over the 1992-2007 period.

Appendix Table E1 shows that the increase in the number of Medicare-Friendly grants funded is greater than the increase in the number of Medicare-Averse or Medicare-Neutral grants funded, though the differences are not statistically significantly different. Taken together, these results document evidence that hospitals that primarily conducted research in conditions affecting elderly populations did not disproportionately benefit from the doubling of the NIH budget. 
Figure E1: Total NIH Grants Funded
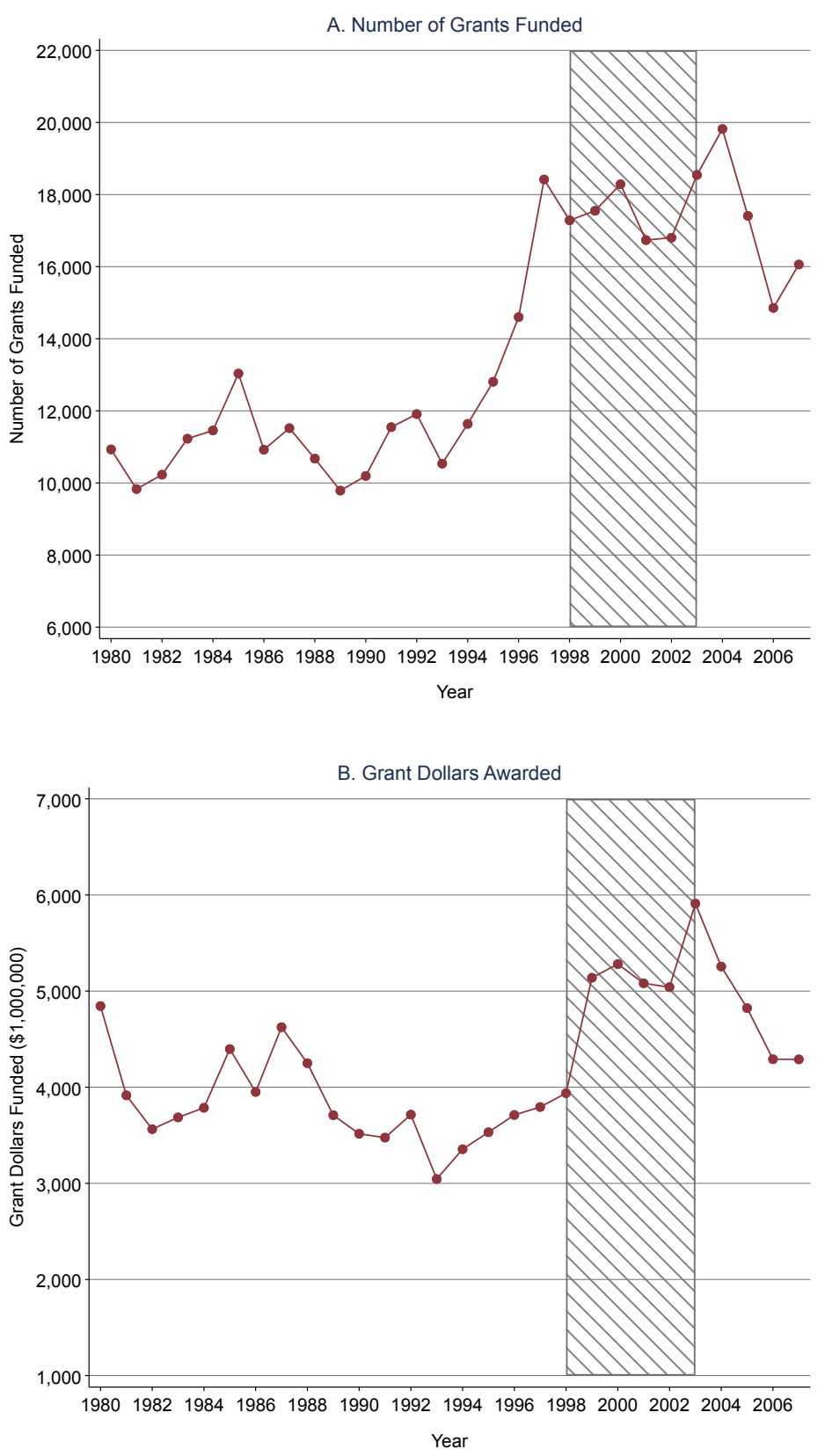

Notes: This figure plots trends in NIH grants by year. This figure is constructed from NIH grants awarded to hospitals and non-hospitals. Panel A plots the total number of grants funded by year. Panel B documents the total amount of NIH grant funds awarded in millions of 1997 dollars. The hatched region from 1998 to 2003 denotes the period in which the NIH budget increased. Source: NIH IMPAC II. 
Figure E2: Total NIH Grants Funded
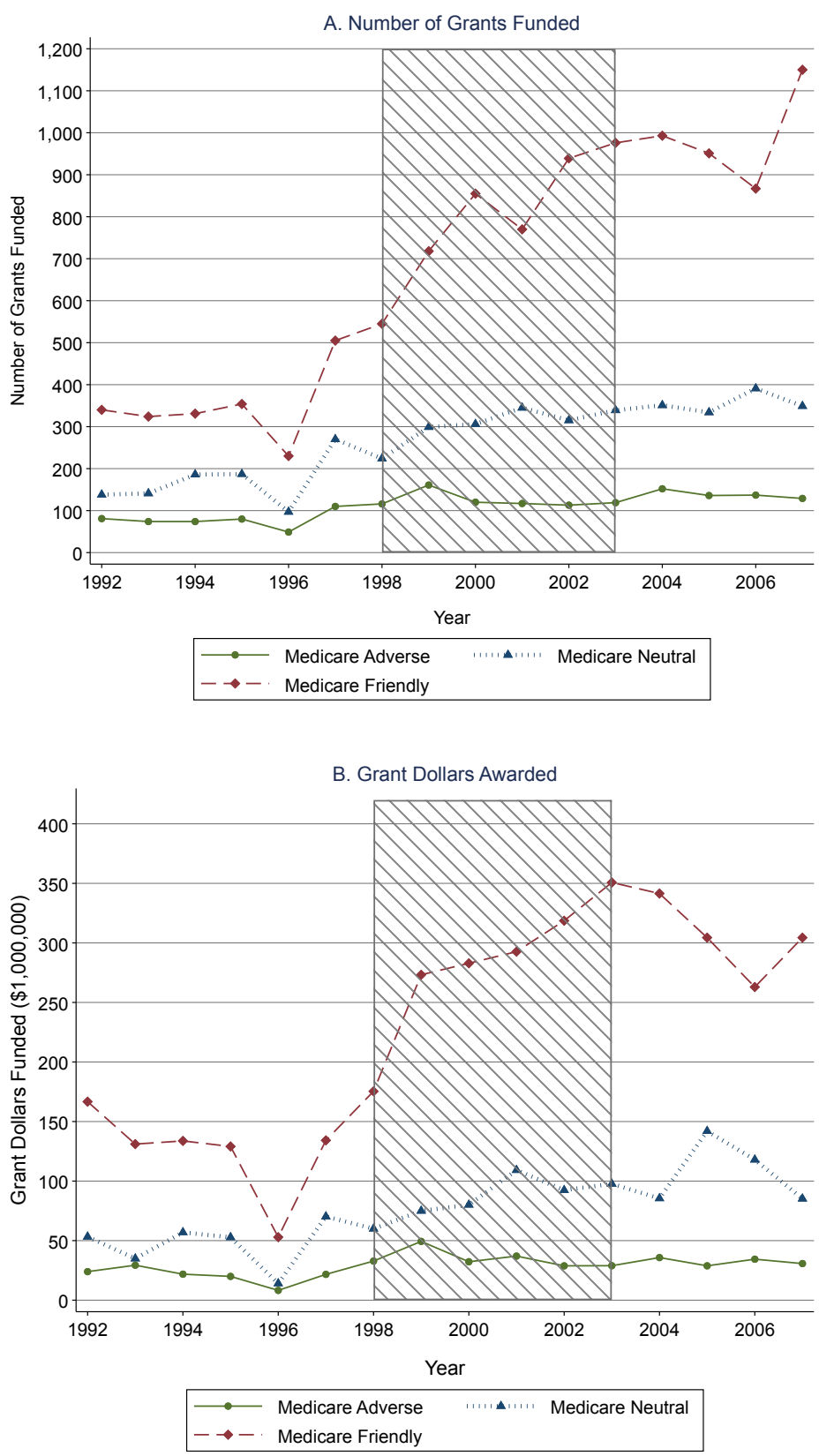

Notes: This figure plots trends in NIH grants by year for three groups of grants: grants that are MedicareAverse, Medicare-Neutral, and Medicare-Friendly. Panel A plots the total number of NIH grants funded by year. Panel B documents the total amount of NIH grant funds awarded in millions of 1997 dollars. The hatched region from 1998 to 2003 denotes the period in which the NIH budget increased. This figure is constructed from only the subset of grants that are linked to the set of MeSH terms that are denoted as Medicare-Averse, Medicare-Neutral, or Medicare-Friendly. The sudden decrease in 1996 is related to our data construction: grants in 1996 were linked to fewer MeSH terms on average (4.12 vs. 9.38 across the whole sample). As a result, grants in 1996 had a lower likelihood of being categorized as Medicare-Averse, Medicare-Neutral, or Medicare-Friendly. For details on the sample and data construction, see the text. Sources: MEPS; NIH IMPAC II. 
Table E1: NIH Budget Increase and Age Bias

\begin{tabular}{lccccccc}
\hline \hline & \multicolumn{3}{c}{ Number of Grants Funded } & & \multicolumn{3}{c}{ Grant Dollars Awarded (\$) } \\
\cline { 2 - 5 } \cline { 7 - 8 } & Medicare & Medicare & Medicare & & Medicare & Medicare & Medicare \\
& Adverse & Neutral & Friendly & & Adverse & Neutral & Friendly \\
& $(1)$ & $(2)$ & $(3)$ & & $(4)$ & $(5)$ & $(6)$ \\
\hline $1(1998$ to 2003$)$ & $0.246^{* *}$ & $0.309^{*}$ & $0.416^{* *}$ & & $0.362^{* *}$ & 0.328 & $0.475^{* *}$ \\
& $(0.122)$ & $(0.158)$ & $(0.195)$ & & $(0.149)$ & $(0.215)$ & $(0.196)$ \\
\hline Mean of Outcome & 5.355 & 6.209 & 7.100 & & 5.355 & 6.209 & 7.100 \\
Nb. Observations & 48 & 48 & 48 & & 48 & 48 & 48 \\
\hline \hline
\end{tabular}

Notes: This table reports estimates of the relationship between the NIH budget increase on age bias among NIH grants. Three types of NIH grants are examined: grants that are Medicare-Averse, Medicare-Neutral, and Medicare-Friendly. Observations are at the year level and estimations are performed over the 1992-2007 period. The sample of grants used in this analysis is constructed from the subset of grants that are linked to the set of MeSH terms that are denoted as Medicare-Averse, Medicare-Neutral, or Medicare-Friendly. Outcomes are transformed with the inverse hyperbolic sine function. Estimates are from seemingly unrelated regressions and each coefficient is from a separate regression. Sources: MEPS; NIH IMPAC II.

$p<0.10,{ }^{* *} p<0.05,{ }^{* * *} p<0.001$. 


\section{Appendix F: Subsidy BBA Bite Results}

This section provides summary statistics and regression results for the subsidy BBA Bite analysis. In this analysis, BBA Bite is calculated as:

$$
\text { BBA_Bite }{ }_{h}=\frac{I M E_{h, 1995}+D S H_{h, 1995}}{\text { TotalRevenue }_{h, 1995}}
$$

where $I M E_{h, 1995}$ is total indirect medical education payments for hospital $h, D S H_{h, 1995}$ is disproportionate share payments for hospital $h$, and TotalRevenue ${ }_{h, 1995}$ is the total patient revenues for hospital $h$. All values correspond to payments in 1995. 
Figure F1: Distribution of BBA Bite

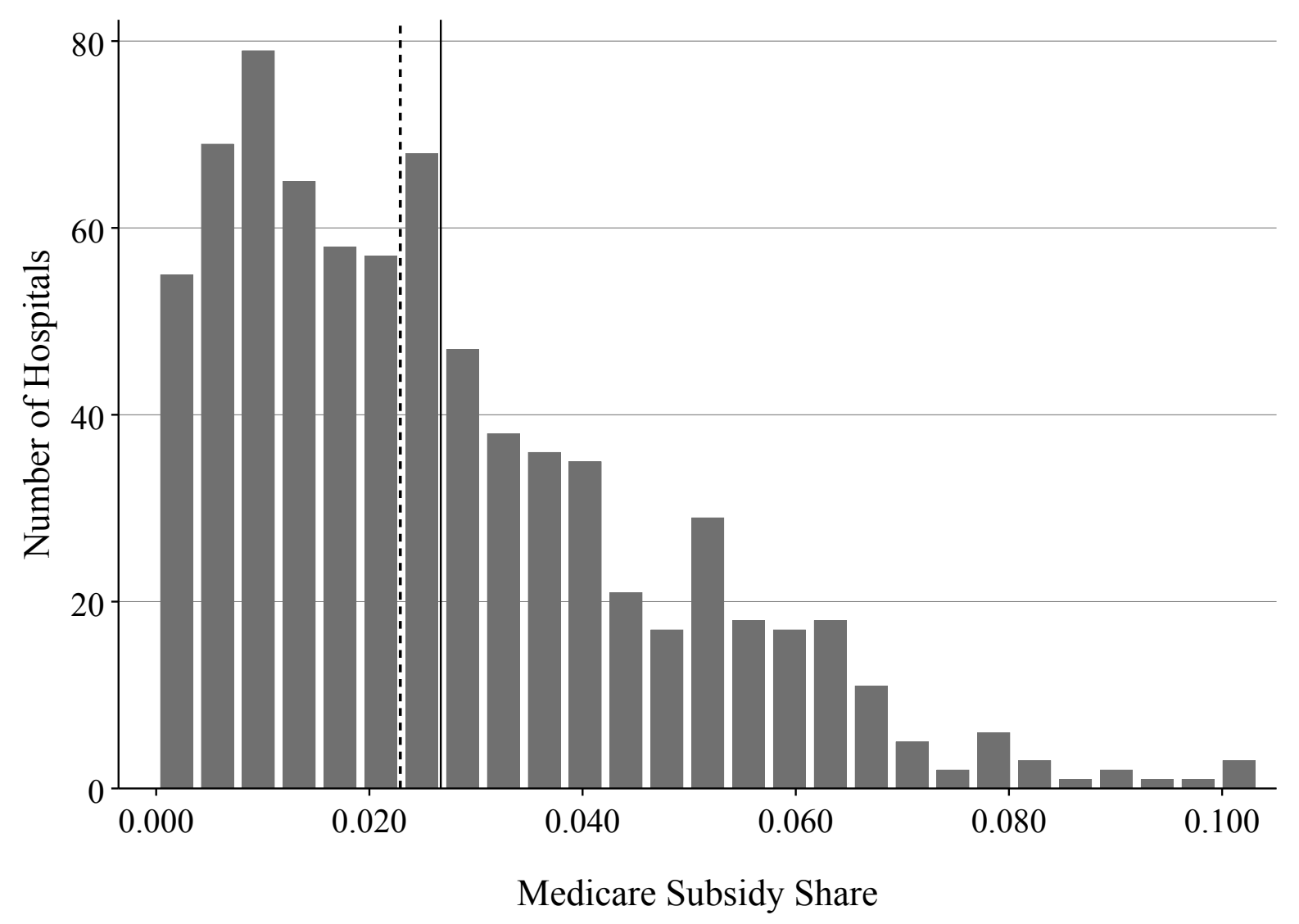

Notes: This figure shows a histogram of the BBA Bite for the teaching hospital sample, where BBA Bite is the share of 1995 patient revenues that come from IME and DSH payments. The solid line indicates the mean (0.0267) of this variable and the dotted lines indicates the median (0.0229). Sources: Healthcare Provider Cost Reporting Information System; Inpatient Prospective Payment System Payment Impact Files. 
Figure F2: Distribution of Research Intensity

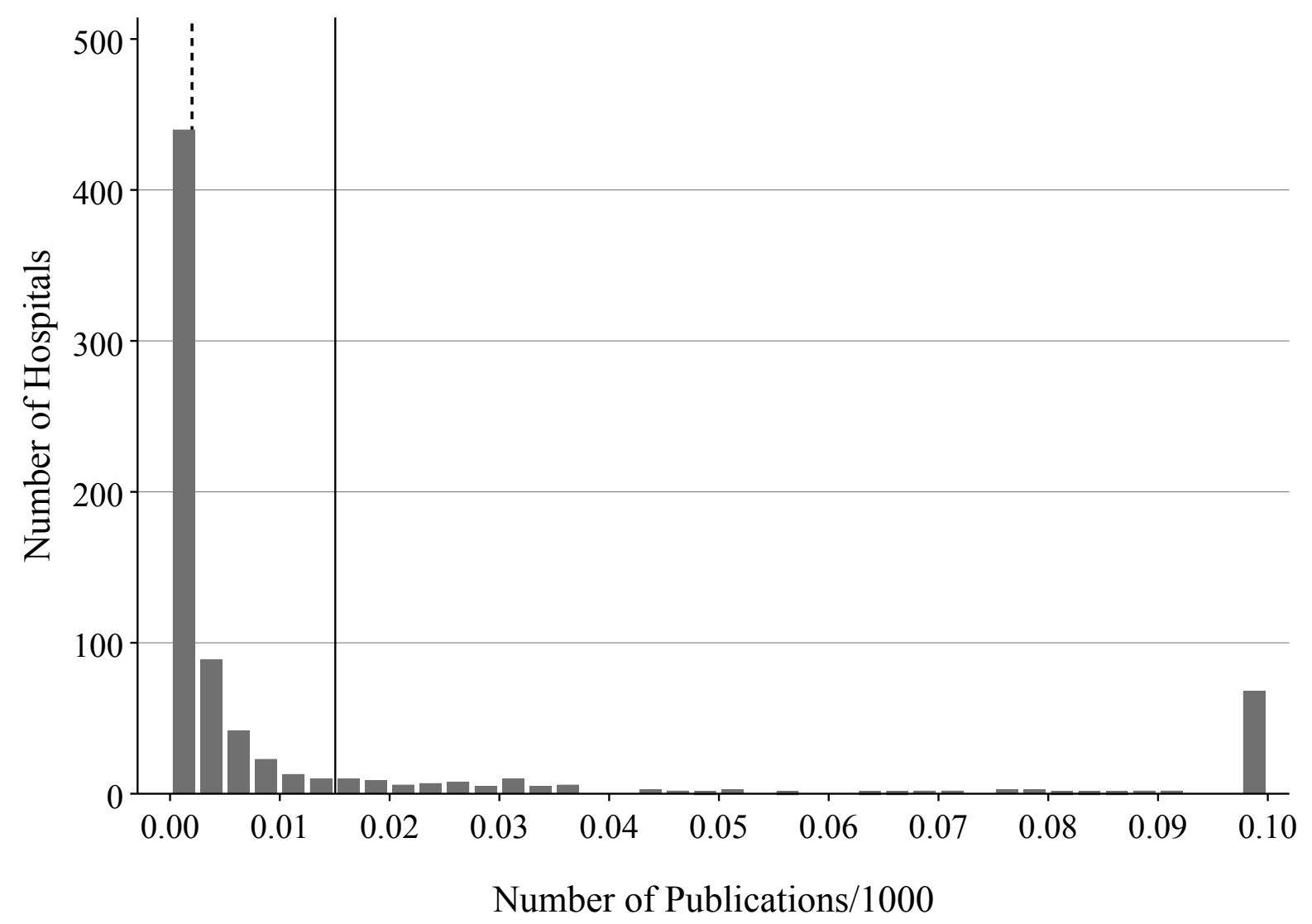

Notes: This figure shows a histogram of the Research Intensity, where Research Intensity is the annual number of publications averaged over 1990-1991 and divided by 1000. The hospital sample used is the teaching hospital sample. The solid line indicates the mean $(0.015)$ of this variable and the dotted lines indicates the median (0.002). For ease of interpretation in this figure, hospitals with Research Intensity greater than 0.3 are recoded as 0.3. Sources: Healthcare Provider Cost Reporting Information System; Inpatient Prospective Payment System Payment Impact Files. 
Figure F3: Effect on the Number of Grant Applications

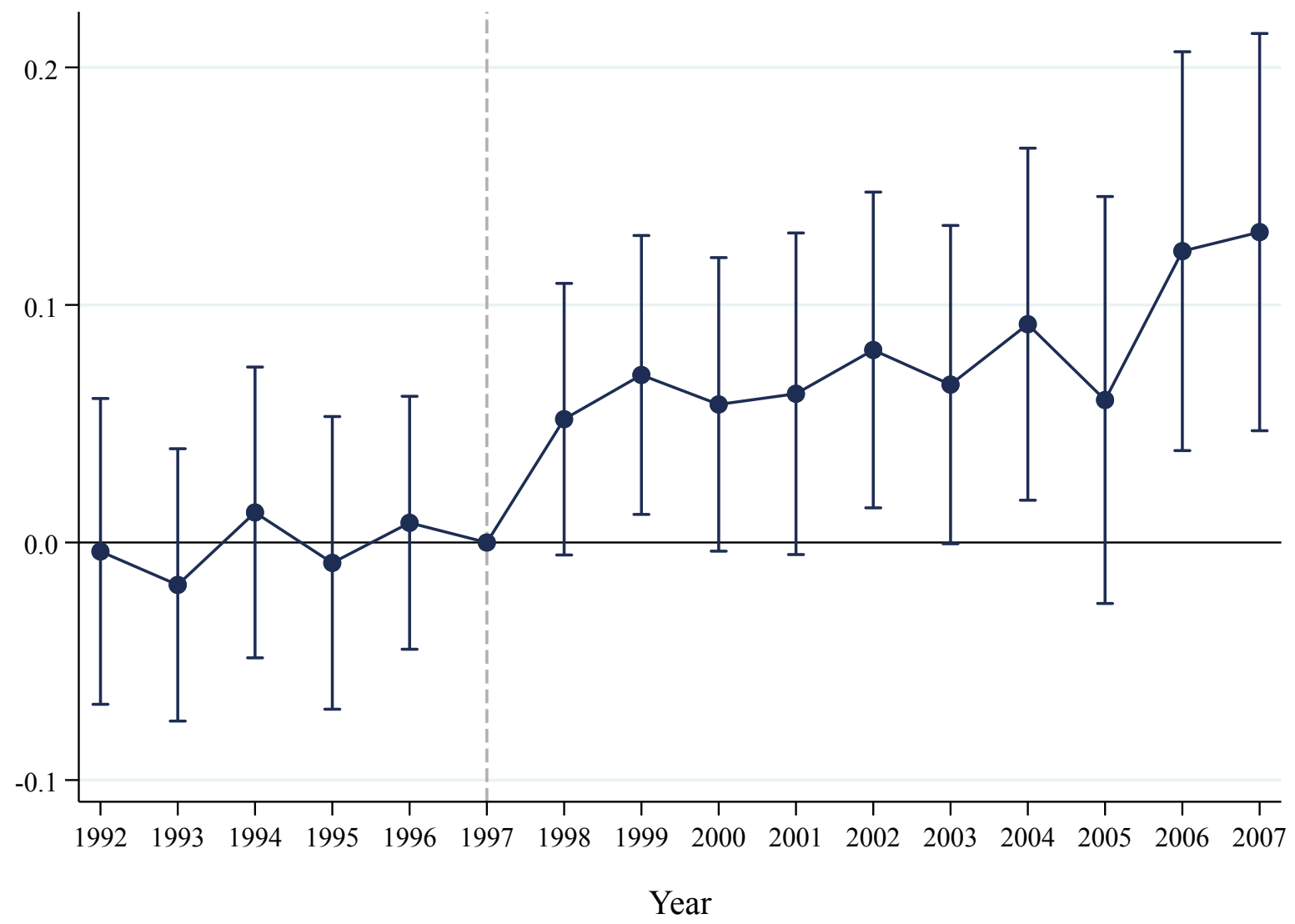

Notes: This figure plots the response in grant applications in the teaching hospital sample, where BBA Bite is the share of 1995 patient revenues that come from IME and DSH payments. Each dot corresponds to OLS coefficient estimates stemming from the event study specification described in Section IV, in which asinh(number of grant applications) is regressed onto year fixed effects, hospital effects, interaction terms between an indicator for a hospital's High BBA Bite status interacted with the number of years before/after the BBA, as well as interaction terms between an indicator for a hospital's High RI status interacted with the number of years before/after the start of the NIH budget doubling. The $95 \%$ confidence interval (corresponding to robust standard errors, clustered around hospital) around these estimates is shown. Sources: Healthcare Provider Cost Reporting Information System; Inpatient Prospective Payment System Payment Impact Files; NIH IMPAC II. 
Figure F4: Effect on the Number of Grants Funded

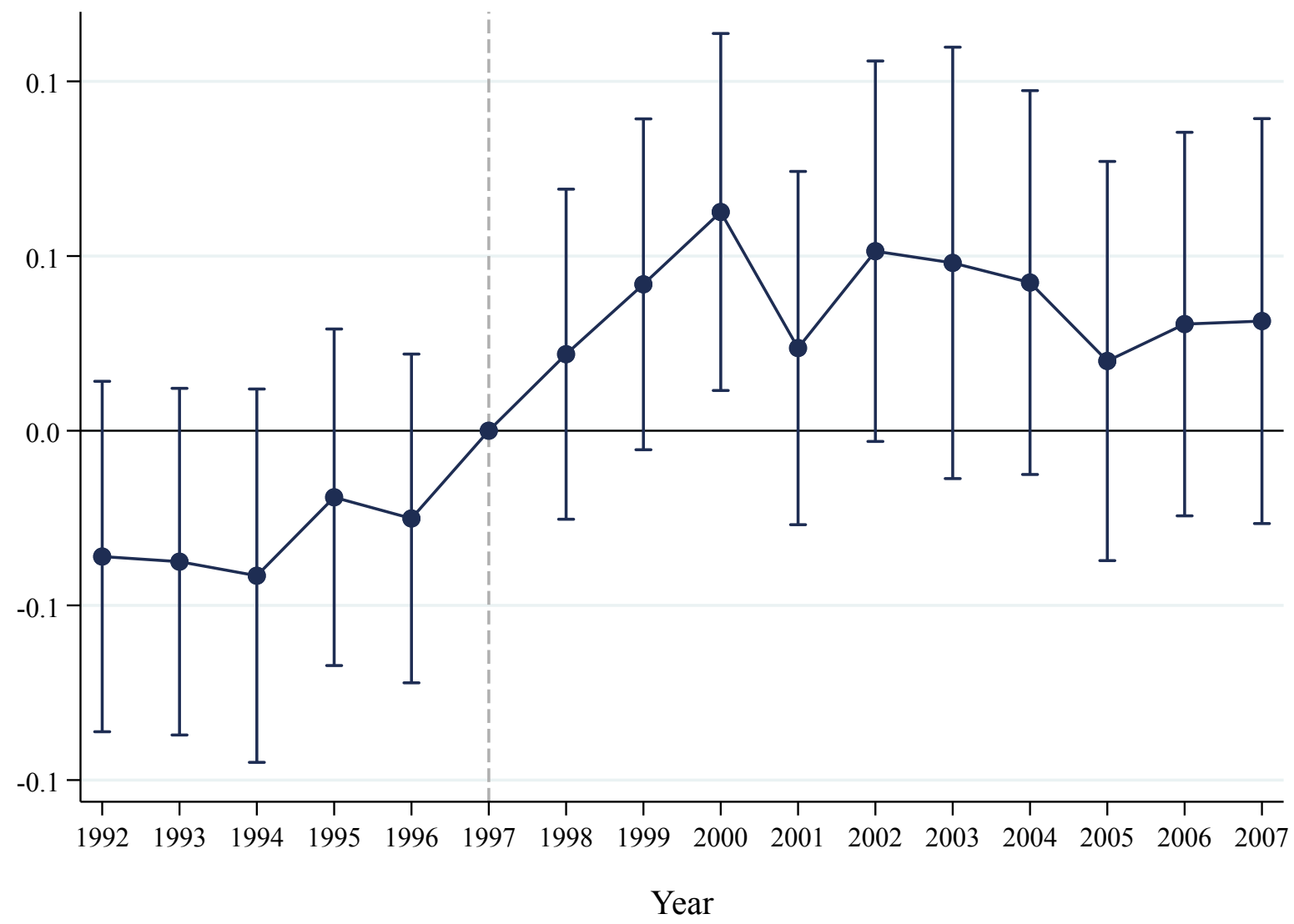

Notes: This figure plots the response in grants funded in the teaching hospital sample, where BBA Bite is the share of 1995 patient revenues that come from IME and DSH payments. Each dot corresponds to OLS coefficient estimates stemming from the event study specification described in Section IV.A, in which asinh(number of grants funded) is regressed onto year fixed effects, hospital effects, interaction terms between an indicator for a hospital's High BBA Bite status interacted with the number of years before/after the BBA, as well as interaction terms between an indicator for a hospital's High RI status interacted with the number of years before/after the start of the NIH budget doubling. The $95 \%$ confidence interval (corresponding to robust standard errors, clustered around hospital) around these estimates is shown. Sources: Healthcare Provider Cost Reporting Information System; Inpatient Prospective Payment System Payment Impact Files; NIH IMPAC II. 
Figure F5: Effect on the Number of Publications

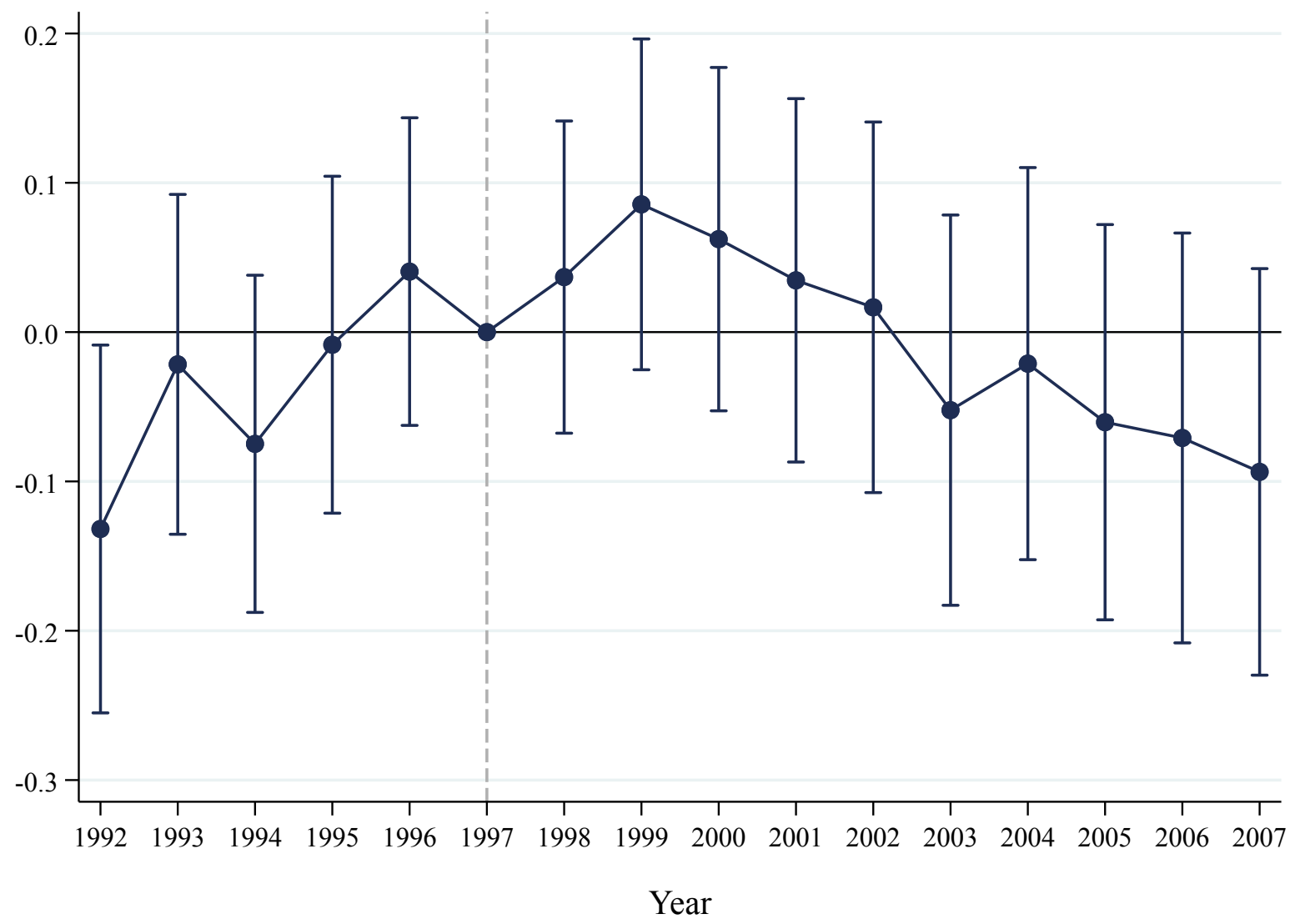

Notes: This figure plots the response in publications in the teaching hospital sample, where BBA Bite is the share of 1995 patient revenues that come from IME and DSH payments. Each dot corresponds to OLS coefficient estimates stemming from the event study specification described in Section IV.A, in which asinh(number of publications) is regressed onto year fixed effects, hospital effects, interaction terms between an indicator for a hospital's High BBA Bite status interacted with the number of years before/after the BBA, as well as interaction terms between an indicator for a hospital's High RI status interacted with the number of years before/after the start of the NIH budget doubling. The $95 \%$ confidence interval (corresponding to robust standard errors, clustered around hospital) around these estimates is shown. Sources: Healthcare Provider Cost Reporting Information System; Inpatient Prospective Payment System Payment Impact Files; PubMed, Web of Science. 
Table F1: Effect on the Number of Grant Applications

\begin{tabular}{|c|c|c|c|c|c|c|c|c|}
\hline & \multirow[b]{2}{*}{$\begin{array}{l}\text { Total } \\
(1)\end{array}$} & \multicolumn{2}{|c|}{ Grant Cycle } & \multicolumn{2}{|c|}{$\begin{array}{c}\text { Principal Investigator } \\
\text { Experience }\end{array}$} & \multicolumn{3}{|c|}{$\begin{array}{c}\text { Principal Investigator } \\
\text { Degree }\end{array}$} \\
\hline & & $\begin{array}{l}\text { New } \\
(2)\end{array}$ & $\begin{array}{c}\text { Renewal } \\
(3)\end{array}$ & $\begin{array}{c}\text { Novice } \\
(4)\end{array}$ & $\begin{array}{c}\text { Incumbent } \\
(5)\end{array}$ & $\begin{array}{c}\mathrm{MD} \\
(6)\end{array}$ & $\begin{array}{c}\mathrm{PhD} \\
(7)\end{array}$ & $\begin{array}{c}\mathrm{MD}-\mathrm{PhD} \\
(8)\end{array}$ \\
\hline BBA Bite $\times$ Post BBA & $\begin{array}{l}1.940^{* *} \\
(0.676)\end{array}$ & $\begin{array}{c}2.336^{* * *} \\
(0.682)\end{array}$ & $\begin{array}{c}0.279 \\
(0.462)\end{array}$ & $\begin{array}{l}4.004^{* * *} \\
(0.751)\end{array}$ & $\begin{array}{l}1.491^{* *} \\
(0.650)\end{array}$ & $\begin{array}{l}1.554^{* *} \\
(0.651)\end{array}$ & $\begin{array}{l}1.889^{* *} \\
(0.575)\end{array}$ & $\begin{array}{c}2.501^{* * *} \\
(0.635)\end{array}$ \\
\hline Elasticity & 0.033 & 0.039 & 0.005 & 0.068 & 0.026 & 0.027 & 0.032 & 0.052 \\
\hline Adjusted $R^{2}$ & 0.040 & 0.057 & 0.010 & 0.129 & 0.025 & 0.033 & 0.067 & 0.100 \\
\hline Diff. Wald test p-value & & \multicolumn{2}{|c|}{0.002} & \multicolumn{2}{|c|}{0.002} & & 0.642 & 0.215 \\
\hline Mean of Outcome & 0.751 & 0.705 & 0.372 & 0.541 & 0.587 & 0.519 & 0.533 & 0.328 \\
\hline Nb. Observations & 12,192 & 12,192 & 12,192 & 12,192 & 12,192 & 12,192 & 12,192 & 12,192 \\
\hline Nb. Hospitals & 762 & 762 & 762 & 762 & 762 & 762 & 762 & 762 \\
\hline
\end{tabular}

Notes: This table reports difference-in-differences estimates of the effect of the BBA on the number of grant applications, in the teaching hospital sample. BBA Bite is the share of 1995 patient revenues that come from IME and DSH payments. Observations are at the hospital-year level. Outcomes are transformed with the inverse hyperbolic sine function. Column 1 presents results from OLS regressions. As shown in equation (3), regressions include hospital fixed effects, year fixed effects, and RI $\times$ Post NIH Doubling, where RI is the average number of publications between 1990-1991 divided by 1000. Columns 2-8 present estimates from seemingly unrelated regressions. Each coefficient is from a separate regression. The elasticity of 0.033 implies that a one percentage increase in BBA Bite is associated on average with a 0.033 percent yearly increase in grant applications following the BBA's enactment. The fourth row and eighth row show $p$-values from Wald tests that compare coefficients on BBA Bite $\times$ Post BBA across different columns (Column 2 vs. Column 3; Column 4 vs. Column 5; Column 6 vs. Column 7; Column 6 vs. Column 8). Robust standard errors (clustered at the hospital level) are in parentheses. Sources: Healthcare Provider Cost Reporting Information System; Inpatient Prospective Payment System Payment Impact Files; NIH IMPAC II.

${ }^{*} p<0.10,{ }^{* *} p<0.05,{ }^{* * *} p<0.001$. 
Table F2: Effect on the Number of Grants Funded

\begin{tabular}{|c|c|c|c|c|c|c|c|c|}
\hline & \multirow[b]{2}{*}{$\begin{array}{l}\text { Total } \\
(1)\end{array}$} & \multicolumn{2}{|c|}{ Grant Cycle } & \multicolumn{2}{|c|}{$\begin{array}{c}\text { Principal Investigator } \\
\text { Experience }\end{array}$} & \multicolumn{3}{|c|}{$\begin{array}{c}\text { Principal Investigator } \\
\text { Degree }\end{array}$} \\
\hline & & $\begin{array}{l}\text { New } \\
(2)\end{array}$ & $\begin{array}{l}\text { Renewal } \\
(3)\end{array}$ & $\begin{array}{l}\text { Novice } \\
\text { (4) }\end{array}$ & $\begin{array}{c}\text { Incumbent } \\
(5)\end{array}$ & $\begin{array}{l}\mathrm{MD} \\
(6)\end{array}$ & $\begin{array}{c}\mathrm{PhD} \\
(7)\end{array}$ & $\begin{array}{c}\text { MD-PhD } \\
\text { (8) }\end{array}$ \\
\hline BBA Bite $\times$ Post BBA & $\begin{array}{l}1.378^{* *} \\
(0.553)\end{array}$ & $\begin{array}{l}1.944^{* * *} \\
(0.589)\end{array}$ & $\begin{array}{c}0.164 \\
(0.344)\end{array}$ & $\begin{array}{c}2.434^{* * *} \\
(0.548)\end{array}$ & $\begin{array}{l}0.830^{*} \\
(0.483)\end{array}$ & $\begin{array}{l}0.789^{*} \\
(0.449)\end{array}$ & $\begin{array}{l}1.338^{* *} \\
(0.569)\end{array}$ & $\begin{array}{l}1.509^{* * *} \\
(0.400)\end{array}$ \\
\hline Elasticity & 0.025 & 0.037 & 0.005 & 0.050 & 0.019 & 0.019 & 0.029 & 0.068 \\
\hline Adjusted $R^{2}$ & 0.038 & 0.060 & 0.015 & 0.113 & 0.018 & 0.033 & 0.058 & 0.085 \\
\hline Diff. Wald test p-value & & \multicolumn{2}{|c|}{0.001} & \multicolumn{2}{|c|}{0.002} & & 0.385 & 0.151 \\
\hline Mean of Outcome & 0.439 & 0.382 & 0.246 & 0.325 & 0.312 & 0.285 & 0.300 & 0.159 \\
\hline Nb. Observations & 12,192 & 12,192 & 12,192 & 12,192 & 12,192 & 12,192 & 12,192 & 12,192 \\
\hline Nb. Hospitals & 762 & 762 & 762 & 762 & 762 & 762 & 762 & 762 \\
\hline
\end{tabular}

Notes: This table reports difference-in-differences estimates of the effect of the BBA on the number of grants funded, in the teaching hospital sample. BBA Bite is the share of 1995 patient revenues that come from IME and DSH payments. Observations are at the hospital-year level. Outcomes are transformed with the inverse hyperbolic sine function. Column 1 presents results from OLS regressions. As shown in equation (3), regressions include hospital fixed effects, year fixed effects, and RI $\times$ Post NIH Doubling, where RI is the average number of publications between 1990-1991 divided by 1000. Columns 2-8 present estimates from seemingly unrelated regressions. Each coefficient is from a separate regression. The elasticity of 0.025 implies that a one percentage increase in BBA Bite is associated on average with a 0.025 percent yearly increase in grants funded following the BBA's enactment. The fourth row and eighth row show $p$-values from Wald tests that compare coefficients on BBA Bite $\times$ Post BBA across different columns (Column 2 vs. Column 3; Column 4 vs. Column 5; Column 6 vs. Column 7; Column 6 vs. Column 8). Robust standard errors (clustered at the hospital level) are in parentheses. Sources: Healthcare Provider Cost Reporting Information System; Inpatient Prospective Payment System Payment Impact Files; NIH IMPAC II. ${ }^{*} p<0.10,{ }^{* *} p<0.05,{ }^{* * *} p<0.001$. 


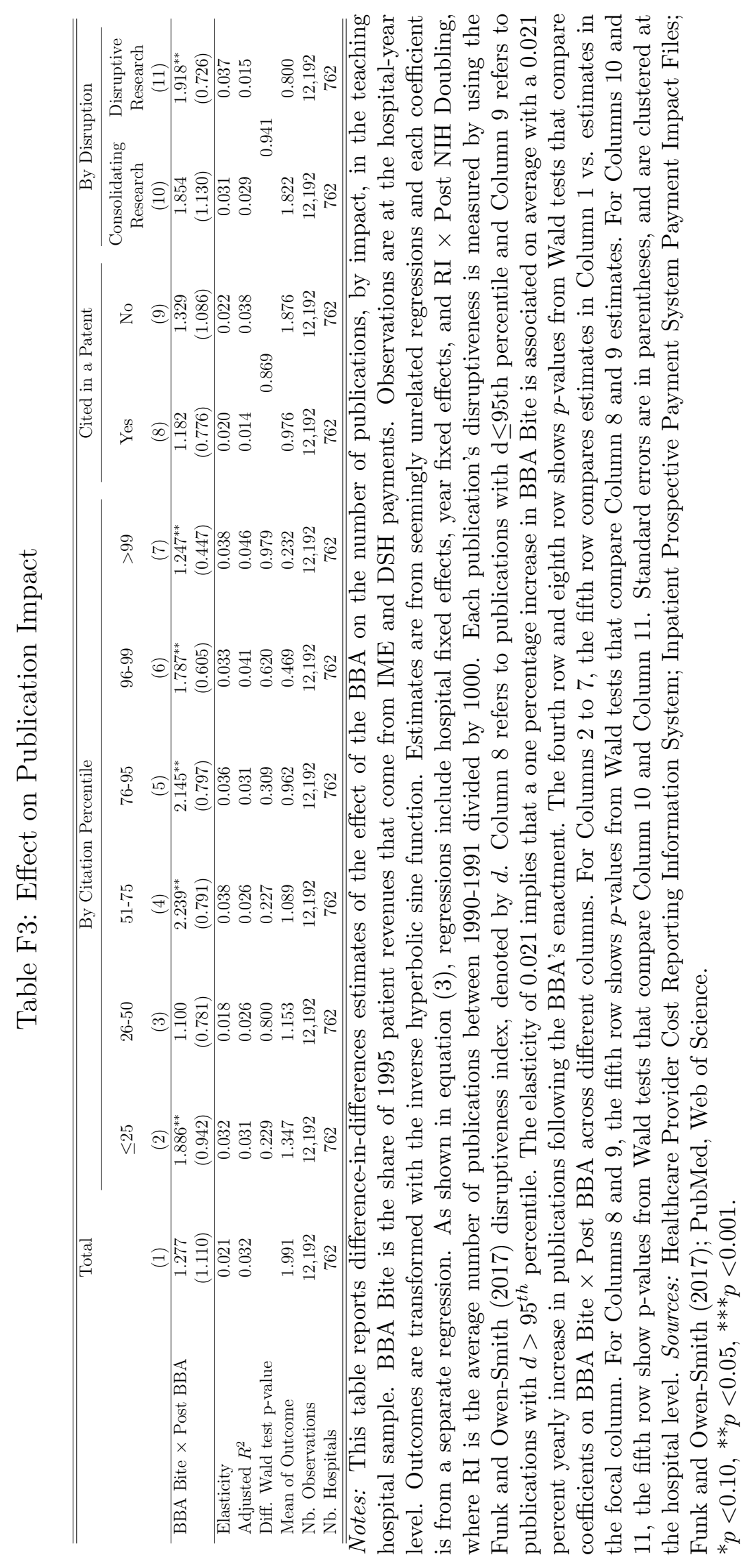

xlvi 
Table F4: Effect on Publication Composition

\begin{tabular}{|c|c|c|c|c|c|c|}
\hline & \multirow{2}{*}{$\begin{array}{c}\begin{array}{c}\text { Laboratory } \\
\text { Research }\end{array} \\
\text { Bench } \\
\text { MeSH }\end{array}$} & \multicolumn{3}{|c|}{ Translational Research } & \multicolumn{2}{|c|}{ Clinical Research } \\
\hline & & $\begin{array}{c}\text { Translational } \\
\text { MeSH } \\
(2)\end{array}$ & $\begin{array}{c}\text { Builds on } \\
\text { Translational } \\
\text { MeSH } \\
(3)\end{array}$ & $\begin{array}{c}\text { Inspiring } \\
\text { Translational } \\
\text { MeSH } \\
(4)\end{array}$ & $\begin{array}{c}\text { Clinical Trials } \\
\text { (5) }\end{array}$ & $\begin{array}{c}\text { Other } \\
(6)\end{array}$ \\
\hline BBA Bite $\times$ Post BBA & $\begin{array}{l}-0.453 \\
(0.850)\end{array}$ & $\begin{array}{l}2.107^{* *} \\
(0.827)\end{array}$ & $\begin{array}{c}2.992^{* * *} \\
(0.785)\end{array}$ & $\begin{array}{c}2.751^{* * *} \\
(0.781)\end{array}$ & $\begin{array}{c}3.220^{* * *} \\
(0.824)\end{array}$ & $\begin{array}{l}2.119^{* *} \\
(0.914)\end{array}$ \\
\hline Elasticity & -0.008 & 0.035 & 0.052 & 0.046 & 0.055 & 0.035 \\
\hline Adjusted $R^{2}$ & 0.001 & 0.022 & 0.080 & 0.034 & 0.097 & 0.028 \\
\hline Mean of Outcome & 0.826 & 1.062 & 0.798 & 0.804 & 0.950 & 1.398 \\
\hline Nb. Observations & 12192 & 12192 & 12192 & 12192 & 12192 & 12192 \\
\hline Nb. Hospitals & 762 & 762 & 762 & 762 & 762 & 762 \\
\hline
\end{tabular}

Notes: This table reports difference-in-differences estimates of the effect of the BBA on bench, translational, and clinical trial research in hospitals, in the teaching hospital sample. BBA Bite is the share of 1995 patient revenues that come from IME and DSH payments. Observations are at the hospital-year level. Outcomes are transformed with the inverse hyperbolic sine function. Estimates are from OLS regressions and each coefficient is from a separate regression. As shown in equation (3), regressions include hospital fixed effects, year fixed effects, and RI $\times$ Post NIH Doubling, where RI is the average number of publications between 1990-1991 divided by 1000. Column 1 refers to publications that are not disease-oriented, are not clinical trial publications, and rely on either a molecular biology technique, a model organism, cellular structures and macromolecules, or biochemical and cellular processes (based on MeSH terms). Column 2 refers to publications that are disease-oriented, are not clinical trial publications, and rely on either a molecular biology technique, a model organism, cellular structures and macromolecules, or biochemical and cellular processes (based on MeSH terms). Column 3 refers to publications that report the results of a clinical trials, or are tagged by a human MeSH term and also cite a translational publication. Column 4 refers to publications that are translational and is cited by a clinical trial publication (or one that contains a human MeSH term). Column 5 refers to publications that are indicated as clinical trials based on MeSH terms or the publication type field in PubMed. Finally, Column 6 refers to publications that are disease-oriented, not clinical trials, and not tagged by any bench MeSH keywords. The elasticity of -0.008 implies that a one percentage increase in BBA Bite is associated on average with a 0.008 percent yearly decrease in publications following the BBA's enactment. Standard errors are in parentheses, and are clustered at the hospital level. Sources: Healthcare Provider Cost Reporting Information System; Inpatient Prospective Payment System Payment Impact Files; Azoulay, Greenblatt, and Heggeness (2021); Marx and Fuegi (2020); PubMed; Web of Science.

${ }^{*} p<0.10,{ }^{* *} p<0.05,{ }^{* * *} p<0.001$. 


\section{Appendix G: Instrumental Variables Estimation}

This section provides regression results for the instrumental variables estimation. As with the BBA Bite specification described in Section III.B, this empirical strategy only captures exogenous variation in the PPS reimbursement formula.

Following Shen (2003), we estimate hospital-level "long-difference" regressions separately for the two periods, 1995-1999 and 1995-2007. For each period, we estimate the following equation:

$$
\begin{aligned}
& \text { Research }_{h, t_{2}}-\text { Research }_{h, 1995}= \\
& \beta\left[\text { ActualRev }_{h, t_{2}}-\text { NoBBARev }_{h, t_{2}}\right] \times\left[\frac{\text { MedicareDischarges }}{\text { TotalDischarges }}\right]_{h, 1995} \\
& +\delta \text { Discharges }_{h, 1995}
\end{aligned}
$$

where Research $h_{h, t_{2}}$ denotes research in hospital $h$ in the final year of the period $t_{2}$, and Discharges $_{h, 1995}$ is the number of discharges in 1995. ActualRev $v_{h, t_{2}}$ is the true PPS revenue per discharge for hospital $h$ in year $t_{2}$. To capture the impact of the BBA, we compute $N o B B A R e v_{h, t_{2}}$, a "no BBA" PPS revenue per discharge which is the revenue that hospital $h$ would have received in $t_{2}$ had there been no changes to the PPS formula. We do this by multiplying the PPS revenue per discharge in 1995 by the inflation rate (i.e., the market basket index) for each year until $t_{2}$ (Cutler 1998). As in Section III.B, we weigh the change in PPS revenue per discharge by the hospital's reliance on Medicare, as measured by the share of discharges that are covered by Medicare. To aid the interpretation of the coefficients, we multiply the weighed difference in the true and "no BBA" PPS revenue per discharge by 1000. Figure G2, Panel A displays the distribution of the resulting variable when $t_{2}=1999$. If the BBA leads to an increase in subsequent research levels, we would expect $\beta<0$.

One concern is that ActualRev,$_{h}-N_{o} B B A R e v_{h, t_{2}}$ may not accurately capture the true financial effect of the BBA if hospitals respond to the reform by manipulating parts of the reimbursement formula - i.e., by changing the average patient case-mix. To identify the true financial effect of the BBA, we calculate a simulated revenue based on the updated PPS reimbursement formula, but with inputs held fixed at the pre-BBA (i.e., 1995) level. Figure G1 shows the relationship between the actual, simulated, and no BBA PPS revenue per discharge and Figure G2, Panel B shows the distribution of the simulated revenue change, weighed by the hospital's Medicare share of discharges. 
With these revenue variables, we estimate the following equation in the first stage:

$$
\begin{aligned}
& {\left[\text { ActualRev }_{h, t_{2}}-\operatorname{NoBBARev}_{h, t_{2}}\right] \times\left[\frac{\text { MedicareDischarges }}{\text { TotalDischarges }}\right]_{h, 1995}=} \\
& \gamma\left[\text { SimRev }_{h, t_{2}}-\operatorname{NoBBARev}_{h, t_{2}}\right] \times\left[\frac{\text { MedicareDischarges }}{\text { TotalDischarges }}\right]_{h, 1995} \\
& +\delta \text { Discharges }_{h, 1995}
\end{aligned}
$$

The 2SLS estimates in Table G1 reports the two-stage least square regression results for our primary research outcomes. The results are consistent with our core results: hospitals that experience a larger financial loss as a result of the BBA experience an increase in research outcomes. The effects are both seen in the short-run (Panel A) and long-run (Panel B), though the effects are only statistically significant for publications in the long-run. 
Figure G1: Trends in Actual, Simulated, and No BBA Medicare Revenues

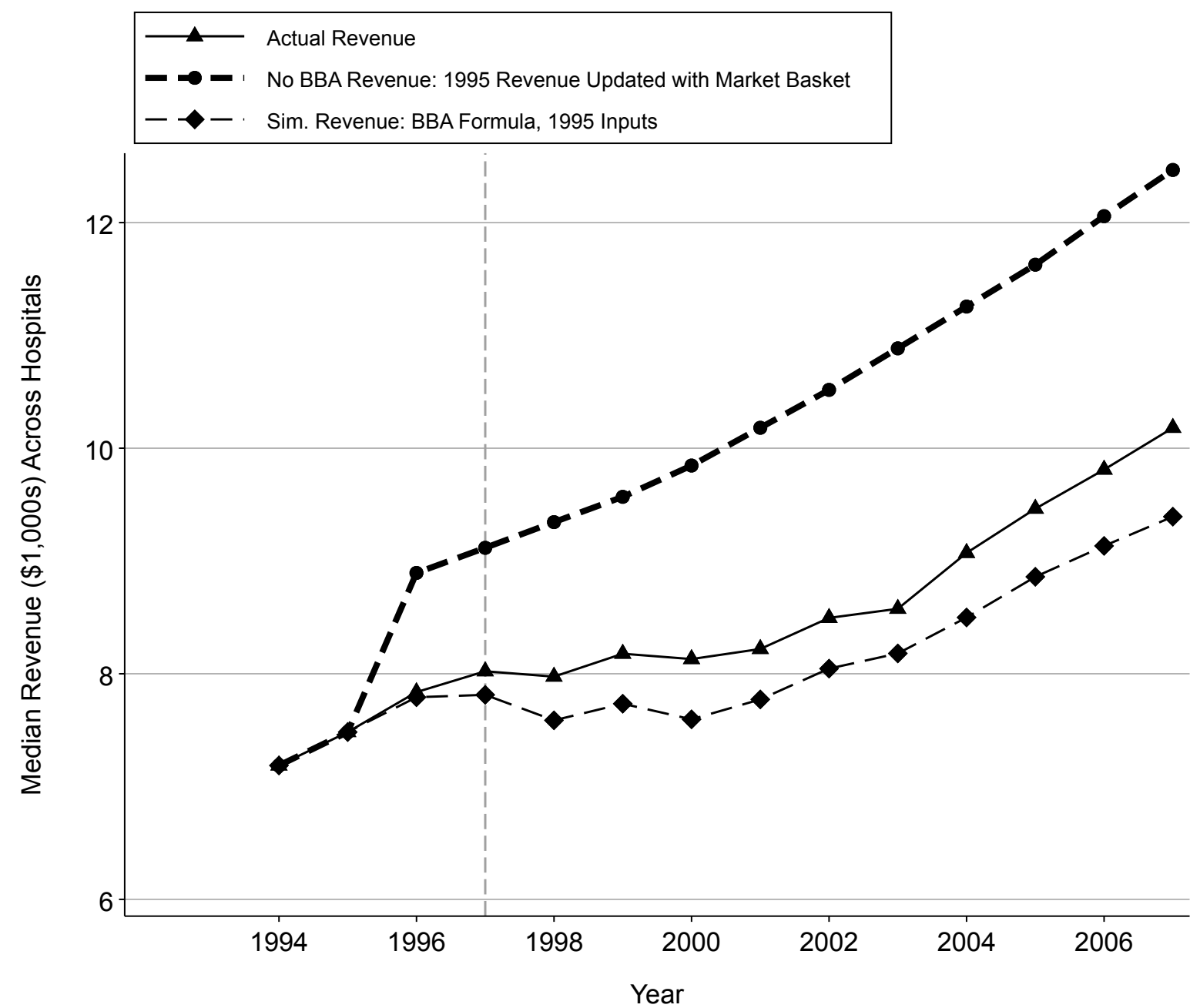

Notes: This figure plots the median level of actual, simulated, and no BBA PPS revenue per discharge, averaged across hospitals in the teaching hospital sample. The dashed line indicates the year in which the BBA came into effect. The dashed line indicates the year in which the BBA came into effect. Sources: Healthcare Provider Cost Reporting Information System, Inpatient Prospective Payment System Payment Impact Files. 


\section{Figure G2: Distribution in Revenue Changes}

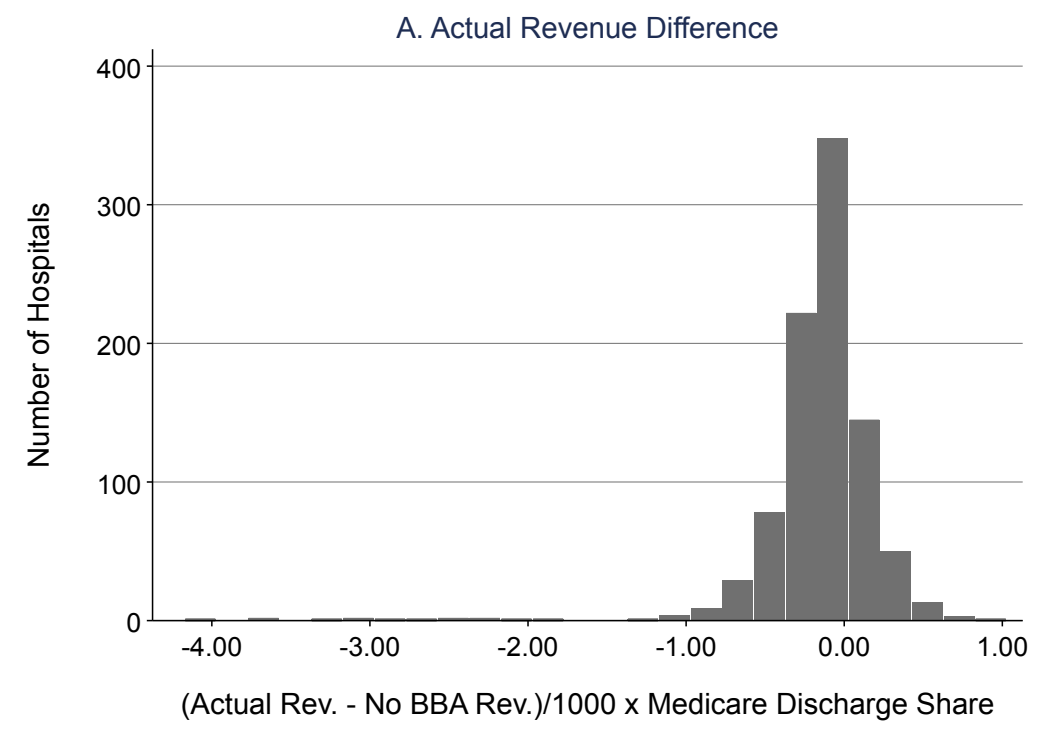

B. Sim. Revenue Difference

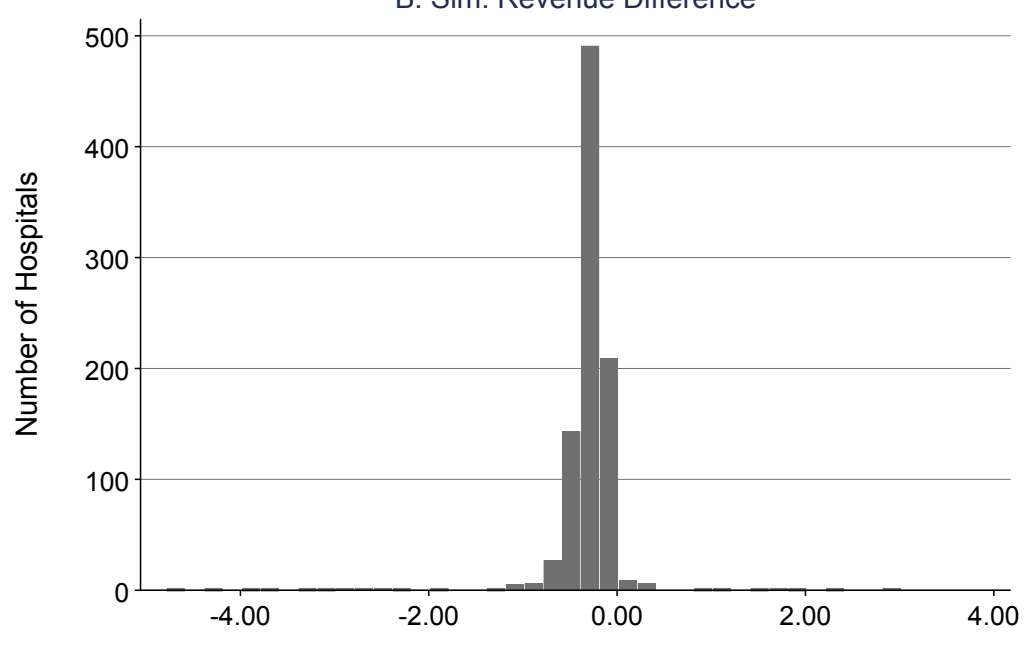

(Sim. Rev. - No BBA Rev.)/1000 x Medicare Discharge Share

Notes: This figure shows a histogram of the true change (Panel A) and the simulated change (Panel B) in average PPS revenue per discharge, weighed by the Medicare share of discharges. The sample is the teaching hospital sample. Sources: Healthcare Provider Cost Reporting Information System, Inpatient Prospective Payment System Payment Impact Files. 
Table G1: Effect on Research Levels

\begin{tabular}{|c|c|c|c|c|c|c|c|}
\hline & \multirow{2}{*}{$\begin{array}{c}\text { First Stage } \\
\Delta \text { Actual Rev. } \\
(1)\end{array}$} & \multicolumn{2}{|c|}{ Grant Applications } & \multicolumn{2}{|c|}{ Grants Funded } & \multicolumn{2}{|c|}{ Total Pubs } \\
\hline & & $\begin{array}{l}\text { OLS } \\
(2)\end{array}$ & $\begin{array}{l}\text { IV } \\
(3)\end{array}$ & $\begin{array}{l}\text { OLS } \\
(4)\end{array}$ & $\begin{array}{l}\text { IV } \\
(5)\end{array}$ & $\begin{array}{l}\text { OLS } \\
(6)\end{array}$ & $\begin{array}{l}\text { IV } \\
(7)\end{array}$ \\
\hline $\begin{array}{l}\text { A. } 1995-1999 \\
\Delta \text { Sim. Rev. }\end{array}$ & $\begin{array}{l}0.713^{* * *} \\
(0.0808)\end{array}$ & & & & & & \\
\hline$\Delta$ True Rev. & & $\begin{array}{c}0.237 \\
(0.371)\end{array}$ & $\begin{array}{c}0.202 \\
(0.449)\end{array}$ & $\begin{array}{l}0.0933 \\
(0.207)\end{array}$ & $\begin{array}{l}-0.193 \\
(0.326)\end{array}$ & $\begin{array}{l}-0.449 \\
(2.506)\end{array}$ & $\begin{array}{l}-5.784 \\
(4.122)\end{array}$ \\
\hline Discharges in 1995 & $\begin{array}{r}-0.000512 \\
(0.00125)\end{array}$ & $\begin{array}{l}0.118^{* * *} \\
(0.0349)\end{array}$ & $\begin{array}{l}0.118^{* * *} \\
(0.0348)\end{array}$ & $\begin{array}{c}0.0877^{* * *} \\
(0.0226)\end{array}$ & $\begin{array}{c}0.0876^{* * *} \\
(0.0225)\end{array}$ & $\begin{array}{c}1.398^{* * *} \\
(0.283)\end{array}$ & $\begin{array}{l}1.395^{* * *} \\
(0.282)\end{array}$ \\
\hline Cragg-Donald Wald F-Stat & 44 & & & & & & \\
\hline Mean of Outcome & -0.17 & 0.98 & 0.98 & 0.78 & 0.78 & 11.95 & 11.95 \\
\hline Observations & 776 & 776 & 776 & 776 & 776 & 776 & 776 \\
\hline $\begin{array}{l}\text { B. } 1995-2007 \\
\Delta \text { Sim. Rev. }\end{array}$ & $\begin{array}{l}0.857^{* * *} \\
(0.0468)\end{array}$ & & & & & & \\
\hline$\Delta$ True Rev. & & $\begin{array}{c}0.321 \\
(1.035)\end{array}$ & $\begin{array}{l}-1.113 \\
(1.404)\end{array}$ & $\begin{array}{c}0.108 \\
(0.185)\end{array}$ & $\begin{array}{l}-0.128 \\
(0.240)\end{array}$ & $\begin{array}{l}-3.619 \\
(3.651)\end{array}$ & $\begin{array}{l}-8.800^{*} \\
(4.747)\end{array}$ \\
\hline Discharges in 1995 & $\begin{array}{l}-0.00382^{*} \\
(0.00213)\end{array}$ & $\begin{array}{c}0.915^{* * *} \\
(0.250)\end{array}$ & $\begin{array}{c}0.897^{* * *} \\
(0.248)\end{array}$ & $\begin{array}{c}0.129^{* *} \\
(0.0463)\end{array}$ & $\begin{array}{c}0.126^{* *} \\
(0.0459)\end{array}$ & $\begin{array}{c}2.601^{* * * *} \\
(0.669)\end{array}$ & $\begin{array}{c}2.534^{* * *} \\
(0.663)\end{array}$ \\
\hline Cragg-Donald Wald F-Stat & 214 & & & & & & \\
\hline Mean of Outcome & -0.48 & 8.78 & 8.78 & 1.30 & 1.30 & 25.69 & 25.69 \\
\hline Observations & 739 & 739 & 739 & 739 & 739 & 739 & 739 \\
\hline
\end{tabular}

Notes: This table reports "long-difference" 2SLS estimates of the effect of the BBA on the number of grant applications, grants funded, and publications, in the teaching hospital sample. Observations are at the hospital-level. Panel A examines the short-run effect of the BBA, by estimating the change in research outcomes between 1995 and 1999. Panel B investigates the long-run effect of the BBA, by estimating the change in research outcomes between 1995 and 2007. For ease of interpretation, "Discharges in 1995" are the number of discharges in 1995 divided by 1000. Robust standard errors are in parentheses. Source: Healthcare Provider Cost Reporting Information System; Inpatient Prospective Payment System Payment Impact Files; NIH IMPAC II; PubMed.

${ }^{*} p<0.10,{ }^{* *} p<0.05,{ }^{* * *} p<0.001$. 


\section{Appendix H: Intended Effects of the BBA}

This appendix provides reports the effect of the BBA on research outcomes where a hospital's BBA Bite is calculated by simulating the revenue per discharge that the hospital would have lost under the BBA, had the intended effects of the reform been in effect in 1995. 
Figure H1: Distribution of BBA Bite

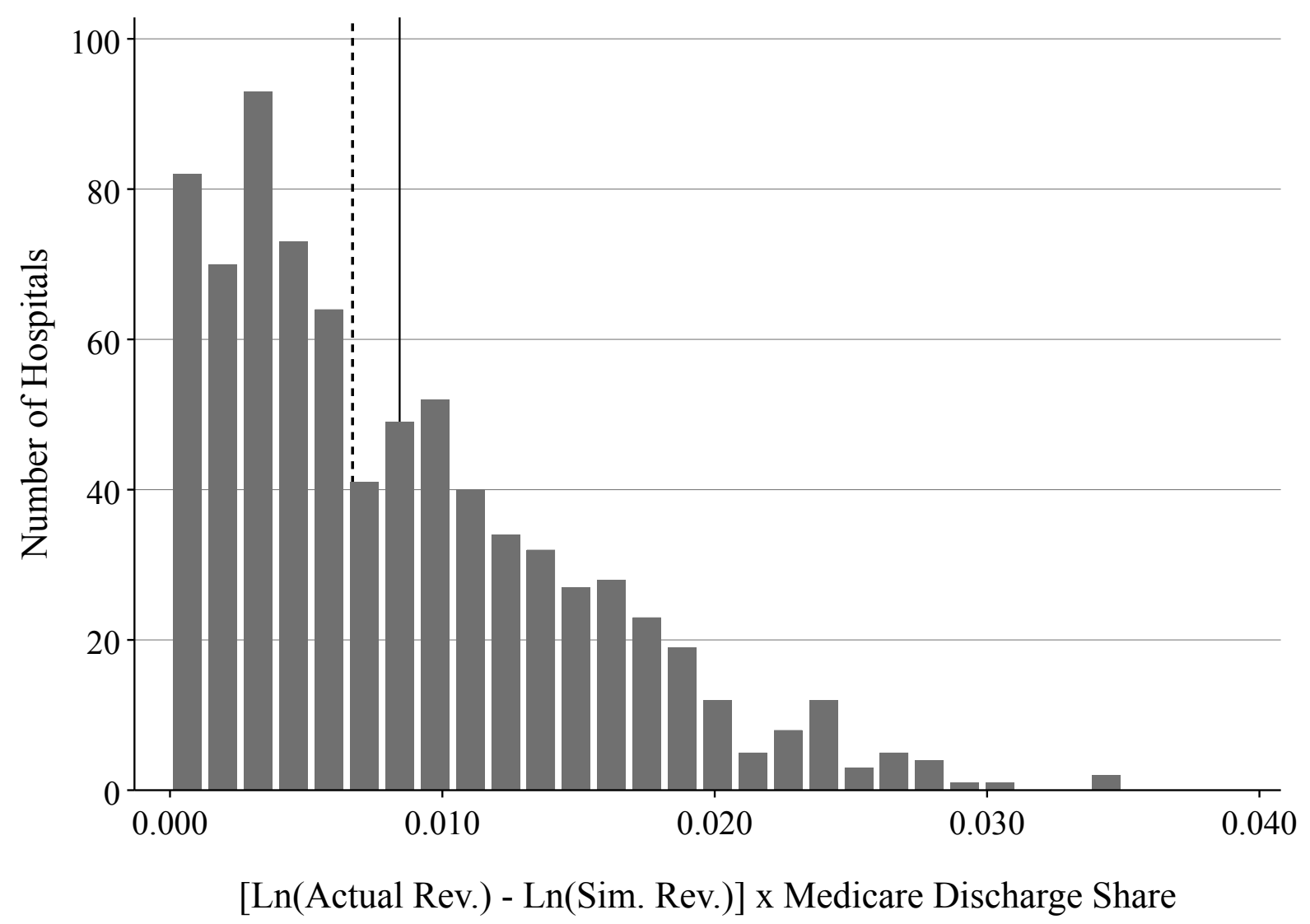

Notes: This figure shows a histogram of the BBA Bite for the teaching hospital sample, where BBA Bite is the product of (i) the difference between the $(\log )$ simulated revenue per discharge and actual (log) PPS revenue per discharge in 1995; and (ii) the Medicare share of discharges averaged over 1992-1995. A hospital's BBA Bite is calculated by simulating the revenue per discharge that the hospital would have lost under the BBA, had the intended effects of the reform been in effect in 1995. The solid line indicates the mean (0.0084) of this variable and the dotted lines indicates the median (0.0067). Sources: Healthcare Provider Cost Reporting Information System; Inpatient Prospective Payment System Payment Impact Files. 
Figure H2: Distribution of Research Intensity

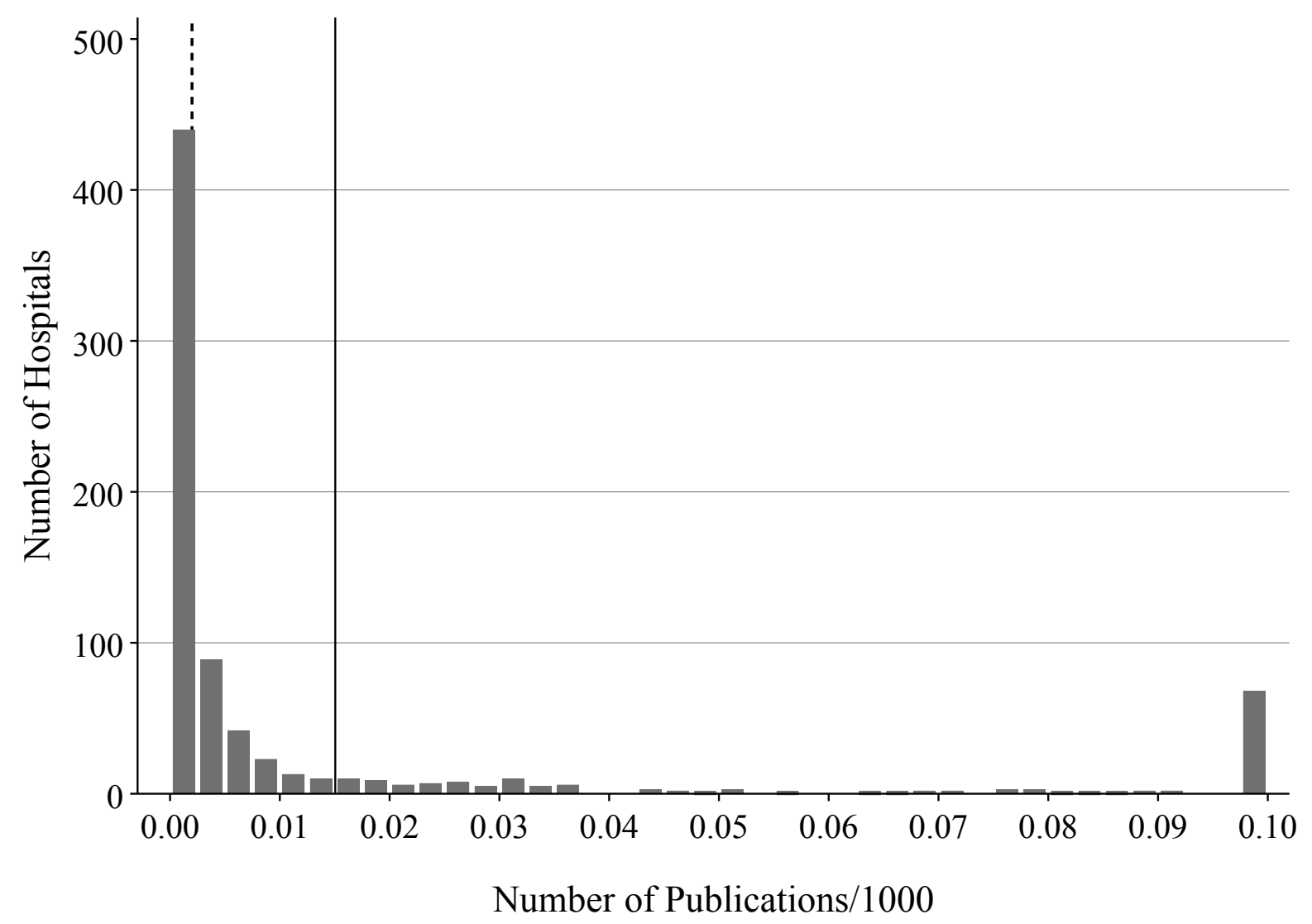

Notes: This figure shows a histogram of the Research Intensity, where Research Intensity is the annual number of publications averaged over 1990-1991 and divided by 1000. The hospital sample used is the teaching hospital sample. The solid line indicates the mean $(0.015)$ of this variable and the dotted lines indicates the median (0.002). For ease of interpretation in this figure, hospitals with Research Intensity greater than 0.3 are recoded as 0.3. Sources: Healthcare Provider Cost Reporting Information System; Inpatient Prospective Payment System Payment Impact Files. 
Figure H3: Effect on the Number of Grant Applications

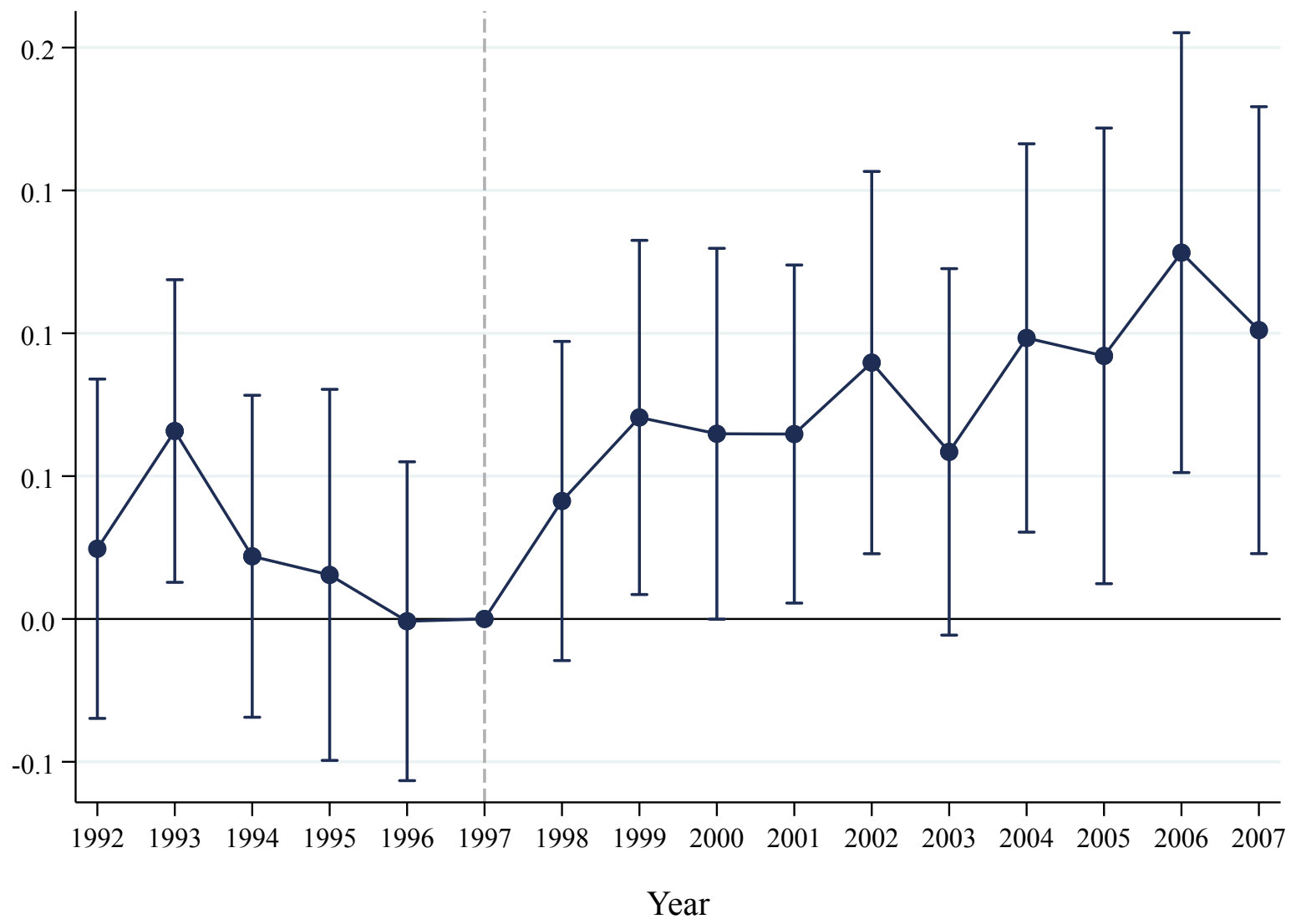

Notes: This figure plots the response in grant applications in the teaching hospital sample. Each dot corresponds to OLS coefficient estimates stemming from the event study specification described in Section IV.A, in which asinh(number of grant applications) is regressed onto year fixed effects, hospital fixed effects, interaction terms between an indicator for a hospital's High BBA Bite status interacted with the number of years before/after the BBA, as well as interaction terms between an indicator for a hospital's High RI status interacted with the number of years before/after the start of the NIH budget doubling. The $95 \%$ confidence interval (corresponding to robust standard errors, clustered around hospital) around these estimates is shown. Sources: Healthcare Provider Cost Reporting Information System; Inpatient Prospective Payment System Payment Impact Files; NIH IMPAC II. 
Figure H4: Effect on the Number of Grants Funded

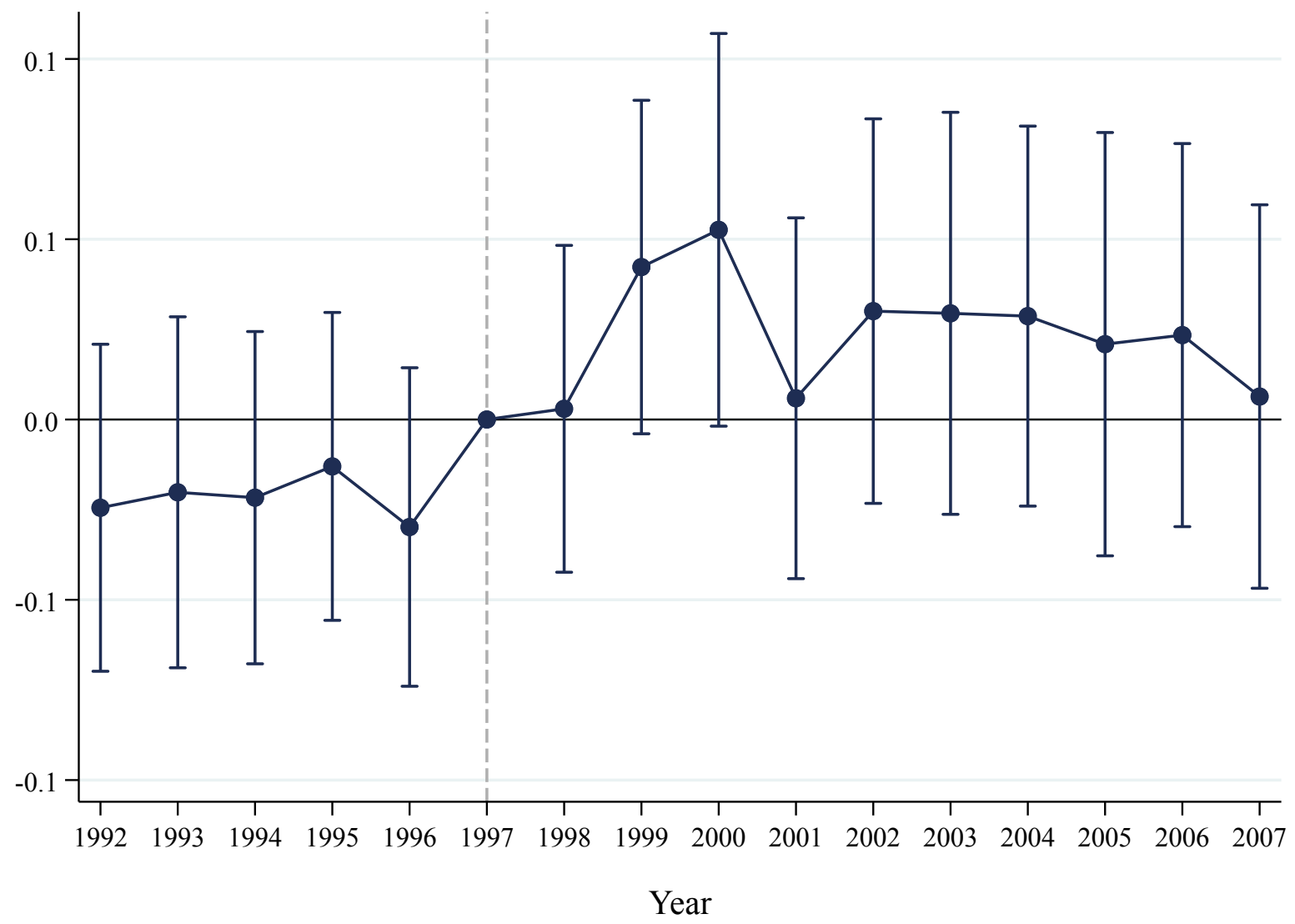

Notes: This figure plots the response in grants funded in the teaching hospital sample. Each dot corresponds to OLS coefficient estimates stemming from the event study specification described in Section IV.A, in which asinh(number of grants funded) is regressed onto year fixed effects, hospital fixed effects, interaction terms between an indicator for a hospital's High BBA Bite status interacted with the number of years before/after the BBA, as well as interaction terms between an indicator for a hospital's High RI status interacted with the number of years before/after the start of the NIH budget doubling. The $95 \%$ confidence interval (corresponding to robust standard errors, clustered around hospital) around these estimates is shown. Sources: Healthcare Provider Cost Reporting Information System; Inpatient Prospective Payment System Payment Impact Files; NIH IMPAC II. 
Figure H5: Effect on the Number of Publications

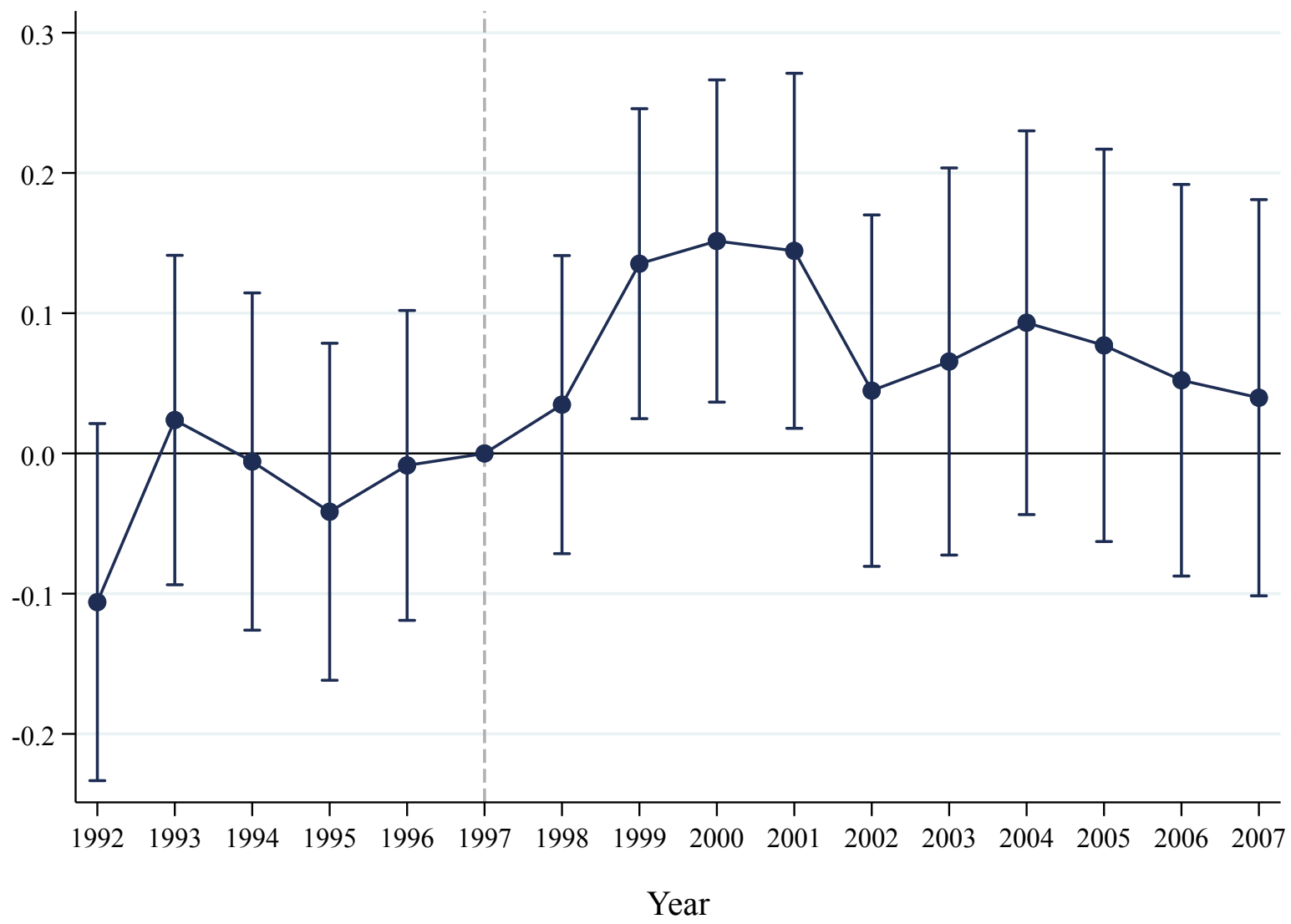

Notes: This figure plots the response in publications in the teaching hospital sample. Each dot corresponds to OLS coefficient estimates stemming from the event study specification described in Section IV.A, in which asinh(number of publications)is regressed onto year fixed effects, hospital fixed effects, interaction terms between an indicator for a hospital's High BBA Bite status interacted with the number of years before/after the BBA, as well as interaction terms between an indicator for a hospital's High RI status interacted with the number of years before/after the start of the NIH budget doubling. The $95 \%$ confidence interval (corresponding to robust standard errors, clustered around hospital) around these estimates is shown. Sources: Healthcare Provider Cost Reporting Information System; Inpatient Prospective Payment System Payment Impact Files; PubMed, Web of Science. 
Table H1: Effect on the Number of Grant Applications

\begin{tabular}{|c|c|c|c|c|c|c|c|c|}
\hline & \multirow[b]{2}{*}{$\begin{array}{c}\text { Total } \\
(1)\end{array}$} & \multicolumn{2}{|c|}{ Grant Cycle } & \multicolumn{2}{|c|}{$\begin{array}{c}\text { Principal Investigator } \\
\text { Experience } \\
\end{array}$} & \multicolumn{3}{|c|}{$\begin{array}{c}\text { Principal Investigator } \\
\text { Degree }\end{array}$} \\
\hline & & $\begin{array}{l}\text { New } \\
(2)\end{array}$ & $\begin{array}{c}\text { Renewal } \\
(3)\end{array}$ & $\begin{array}{l}\text { Novice } \\
(4)\end{array}$ & $\begin{array}{l}\text { Incumbent } \\
(5)\end{array}$ & $\begin{array}{l}\mathrm{MD} \\
(6)\end{array}$ & $\begin{array}{c}\mathrm{PhD} \\
(7)\end{array}$ & $\begin{array}{l}\text { MD-PhD } \\
\quad(8)\end{array}$ \\
\hline BBA Bite $\times$ Post BBA & $\begin{array}{l}4.993^{* *} \\
(2.388)\end{array}$ & $\begin{array}{l}7.006^{* *} \\
(2.445)\end{array}$ & $\begin{array}{l}-0.258 \\
(1.511)\end{array}$ & $\begin{array}{l}14.44^{* * *} \\
(2.377)\end{array}$ & $\begin{array}{l}1.978 \\
(2.157)\end{array}$ & $\begin{array}{l}4.640^{* *} \\
(2.354)\end{array}$ & $\begin{array}{l}6.288^{* *} \\
(2.217)\end{array}$ & $\begin{array}{l}7.815^{* * *} \\
(2.015)\end{array}$ \\
\hline Elasticity & 0.026 & 0.037 & -0.002 & 0.078 & 0.011 & 0.026 & 0.034 & 0.051 \\
\hline Adjusted $R^{2}$ & 0.039 & 0.057 & 0.010 & 0.135 & 0.024 & 0.033 & 0.068 & 0.101 \\
\hline Diff. Wald test p-value & & \multicolumn{2}{|c|}{0.001} & \multicolumn{2}{|c|}{0.000} & & 0.509 & 0.232 \\
\hline Mean of Outcome & 0.751 & 0.705 & 0.372 & 0.541 & 0.587 & 0.519 & 0.533 & 0.328 \\
\hline Nb. Observations & 12,480 & 12,480 & 12,480 & 12,480 & 12,480 & 12,480 & 12,480 & 12,480 \\
\hline Nb. Hospitals & 780 & 780 & 780 & 780 & 780 & 780 & 780 & 780 \\
\hline
\end{tabular}

Notes: This table reports difference-in-differences estimates of the effect of the BBA on the number of grant applications, in the teaching hospital sample. A hospital's BBA Bite is calculated by simulating the revenue per discharge that the hospital would have lost under the BBA, had the intended effects of the reform been in effect in 1995. Observations are at the hospital-year level. Outcomes are transformed with the inverse hyperbolic sine function. Column 1 presents results from OLS regressions. As shown in equation (3), regressions include hospital fixed effects, year fixed effects, and RI $\times$ Post NIH Doubling, where RI is the average number of publications between 1990-1991 divided by 1000. Columns 2-8 present estimates from seemingly unrelated regressions. Each coefficient is from a separate regression. The elasticity of 0.026 implies that a one percentage increase in BBA Bite is associated on average with a 0.026 percent yearly increase in grant applications following the BBA's enactment. The fourth row and eighth row show $p$-values from Wald tests that compare coefficients on BBA Bite $\times$ Post BBA across different columns (Column 2 vs. Column 3; Column 4 vs. Column 5; Column 6 vs. Column 7; Column 6 vs. Column 8). Robust standard errors (clustered at the hospital level) are in parentheses. Sources: Healthcare Provider Cost Reporting Information System; Inpatient Prospective Payment System Payment Impact Files; NIH IMPAC II. ${ }^{*} p<0.10,{ }^{* *} p<0.05,{ }^{* * *} p<0.001$. 
Table H2: Effect on the Number of Grants Funded

\begin{tabular}{|c|c|c|c|c|c|c|c|c|}
\hline & \multirow[b]{2}{*}{$\begin{array}{l}\text { Total } \\
(1)\end{array}$} & \multicolumn{2}{|c|}{ Grant Cycle } & \multicolumn{2}{|c|}{$\begin{array}{c}\text { Principal Investigator } \\
\text { Experience }\end{array}$} & \multicolumn{3}{|c|}{$\begin{array}{c}\text { Principal Investigator } \\
\text { Degree }\end{array}$} \\
\hline & & $\begin{array}{l}\text { New } \\
(2)\end{array}$ & $\begin{array}{c}\text { Renewal } \\
(3)\end{array}$ & $\begin{array}{c}\text { Novice } \\
(4)\end{array}$ & $\begin{array}{c}\text { Incumbent } \\
(5)\end{array}$ & $\begin{array}{l}\mathrm{MD} \\
(6)\end{array}$ & $\begin{array}{c}\mathrm{PhD} \\
(7)\end{array}$ & $\begin{array}{c}\mathrm{MD}-\mathrm{PhD} \\
(8)\end{array}$ \\
\hline BBA Bite $\times$ Post BBA & $\begin{array}{l}3.842^{* *} \\
(1.825)\end{array}$ & $\begin{array}{l}5.502^{* *} \\
(1.813)\end{array}$ & $\begin{array}{l}1.363 \\
(1.327)\end{array}$ & $\begin{array}{l}9.794^{* * *} \\
(1.726)\end{array}$ & $\begin{array}{c}0.834 \\
(1.586)\end{array}$ & $\begin{array}{l}3.038^{*} \\
(1.677)\end{array}$ & $\begin{array}{l}4.555^{* *} \\
(1.646)\end{array}$ & $\begin{array}{c}5.858^{* * *} \\
(1.426)\end{array}$ \\
\hline Elasticity & 0.022 & 0.033 & 0.012 & 0.063 & 0.006 & 0.023 & 0.032 & 0.084 \\
\hline Adjusted $R^{2}$ & 0.038 & 0.060 & 0.015 & 0.119 & 0.017 & 0.034 & 0.059 & 0.087 \\
\hline Diff. Wald test p-value & & 0.010 & & 0.000 & & & 0.399 & 0.143 \\
\hline Mean of Outcome & 0.439 & 0.382 & 0.246 & 0.325 & 0.312 & 0.285 & 0.300 & 0.159 \\
\hline Nb. Observations & 12,480 & 12,480 & 12,480 & 12,480 & 12,480 & 12,480 & 12,480 & 12,480 \\
\hline Nb. Hospitals & 780 & 780 & 780 & 780 & 780 & 780 & 780 & 780 \\
\hline
\end{tabular}

Notes: This table reports difference-in-differences estimates of the effect of the BBA on the number of grants funded, in the teaching hospital sample. A hospital's BBA Bite is calculated by simulating the revenue per discharge that the hospital would have lost under the BBA, had the intended effects of the reform been in effect in 1995. Observations are at the hospital-year level. Outcomes are transformed with the inverse hyperbolic sine function. Column 1 presents results from OLS regressions. As shown in equation (3), regressions include hospital fixed effects, year fixed effects, and RI $\times$ Post NIH Doubling, where RI is the average number of publications between 1990-1991 divided by 1000. Columns 2-8 present estimates from seemingly unrelated regressions. Each coefficient is from a separate regression. The elasticity of 0.022 implies that a one percentage increase in BBA Bite is associated on average with a 0.022 percent yearly increase in grants funded following the BBA's enactment. The fourth row and eighth row show $p$-values from Wald tests that compare coefficients on BBA Bite $\times$ Post BBA across different columns (Column 2 vs. Column 3; Column 4 vs. Column 5; Column 6 vs. Column 7; Column 6 vs. Column 8). Robust standard errors (clustered at the hospital level) are in parentheses. Sources: Healthcare Provider Cost Reporting Information System; Inpatient Prospective Payment System Payment Impact Files; NIH IMPAC II. ${ }^{*} p<0.10,{ }^{* *} p<0.05,{ }^{* * *} p<0.001$. 


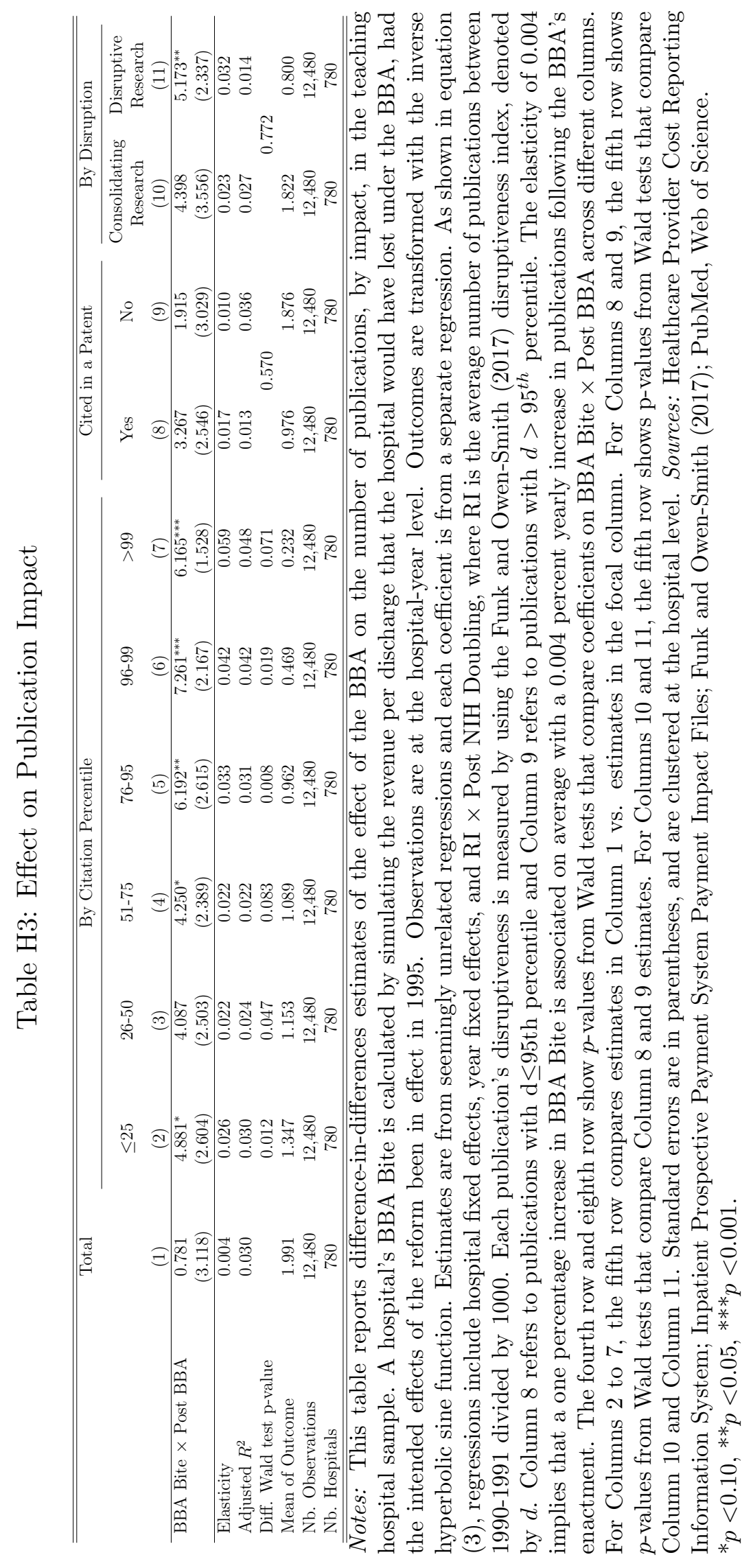


Table H4: Effect on Publication Composition

\begin{tabular}{|c|c|c|c|c|c|c|}
\hline & \multirow{2}{*}{$\begin{array}{c}\begin{array}{c}\text { Laboratory } \\
\text { Research }\end{array} \\
\text { Bench } \\
\text { MeSH }\end{array}$} & \multicolumn{3}{|c|}{ Translational Research } & \multicolumn{2}{|c|}{ Clinical Research } \\
\hline & & $\begin{array}{c}\text { Translational } \\
\text { MeSH } \\
(2)\end{array}$ & $\begin{array}{c}\text { Builds on } \\
\text { Translational } \\
\text { MeSH } \\
(3)\end{array}$ & $\begin{array}{c}\text { Inspiring } \\
\text { Translational } \\
\text { MeSH } \\
(4)\end{array}$ & $\begin{array}{l}\text { Clinical Trials } \\
\qquad(5)\end{array}$ & $\begin{array}{l}\text { Other } \\
(6)\end{array}$ \\
\hline BBA Bite $\times$ Post BBA & $\begin{array}{l}-3.228 \\
(2.765)\end{array}$ & $\begin{array}{l}4.579^{*} \\
(2.521)\end{array}$ & $\begin{array}{c}11.929^{* * *} \\
(2.310)\end{array}$ & $\begin{array}{l}7.381^{* *} \\
(2.368)\end{array}$ & $\begin{array}{c}12.967^{* * *} \\
(2.463)\end{array}$ & $\begin{array}{l}5.593^{* *} \\
(2.695)\end{array}$ \\
\hline Elasticity & -0.017 & 0.024 & 0.065 & 0.039 & 0.069 & 0.030 \\
\hline Adjusted $R^{2}$ & 0.001 & 0.020 & 0.080 & 0.032 & 0.098 & 0.027 \\
\hline Mean of Outcome & 0.826 & 1.062 & 0.798 & 0.804 & 0.950 & 1.398 \\
\hline Nb. Observations & 12480 & 12480 & 12480 & 12480 & 12480 & 12480 \\
\hline Nb. Hospitals & 780 & 780 & 780 & 780 & 780 & 780 \\
\hline
\end{tabular}

Notes: This table reports difference-in-differences estimates of the effect of the BBA on bench, translational, and clinical trial research in hospitals, in the teaching hospital sample. A hospital's BBA Bite is calculated by simulating the revenue per discharge that the hospital would have lost under the BBA, had the intended effects of the reform been in effect in 1995. Observations are at the hospital-year level. Outcomes are transformed with the inverse hyperbolic sine function. Estimates are from OLS regressions and each coefficient is from a separate regression. As shown in equation (3), regressions include hospital fixed effects, year fixed effects, and RI $\times$ Post NIH Doubling, where RI is the average number of publications between 1990-1991 divided by 1000. Column 1 refers to publications that are not disease-oriented, are not clinical trial publications, and rely on either a molecular biology technique, a model organism, cellular structures and macromolecules, or biochemical and cellular processes (based on MeSH terms). Column 2 refers to publications that are disease-oriented, are not clinical trial publications, and rely on either a molecular biology technique, a model organism, cellular structures and macromolecules, or biochemical and cellular processes (based on MeSH terms). Column 3 refers to publications that report the results of a clinical trials, or are tagged by a human MeSH term and also cite a translational publication. Column 4 refers to publications that are translational and is cited by a clinical trial publication (or one that contains a human MeSH term). Column 5 refers to publications that are indicated as clinical trials based on MeSH terms or the publication type field in PubMed. Finally, Column 6 refers to publications that are disease-oriented, not clinical trials, and not tagged by any bench MeSH keywords. The elasticity of -0.017 implies that a one percentage increase in BBA Bite is associated on average with a 0.017 percent yearly decrease in publications following the BBA's enactment. Standard errors are in parentheses, and are clustered at the hospital level. Sources: Healthcare Provider Cost Reporting Information System; Inpatient Prospective Payment System Payment Impact Files; Azoulay, Greenblatt, and Heggeness (2021); Marx and Fuegi (2020); PubMed; Web of Science. ${ }^{*} p<0.10,{ }^{* *} p<0.05,{ }^{* * *} p<0.001$. 


\section{Appendix References}

Cutler, David M. 1998. "Cost Shifting or Cost Cutting? The Incidence of Reductions in Medicare Payments." Tax Policy and the Economy 12: 1-27.

Fishman, Linda E. 1993. How the Medicare Program Pays Hospitals for Inpatient Services: A Primer on the Prospective Payment System. Washington, DC: Association of American Medical Colleges.

Keeler, Emmett B., Grace M. Carter, and Sally Trude. 1988. "Insurance Aspect of DRG Outlier

Payments." Journal of Health Economics 7(3): 193-214

Shen, Yu-Chu. 2003. "The Effect of Financial Pressure on the Quality of Care in Hospitals." Journal of Health Economics 22(2): 243-269.

Wu, Vivian. 2010. "Hospital Cost-shifting Revisited: New Evidence from the Balanced Budget Act of 1997." International Journal of Health Care Finance and Economics 28(2): 350-360. 